\title{
Navigation and Manipulation for Autonomous Underwater Dismantling of Offshore Structures
}

by

\section{Christian Livingston Welch}

B.S., Massachusetts Institute of Technology (2013)

Submitted to the Department of Mechanical Engineering in partial fulfillment of the requirements for the degree of

Master of Science in Mechanical Engineering

at the

MASSACHUSETTS INSTITUTE OF TECHNOLOGY

September 2015

ARCHIVES

MASSACHUSETSINSITUTE
OF TECHNOLOGY
OCT 012015
LIBRARIES

(C)Christian Livingston Welch 2015. All rights reserved.

The author hereby grants to MIT and WHOI permission to reproduce and to distribute publicly copies of this thesis document in whole or in part in any medium now known or hereafter created.

Author

\section{Signature redacted}

Department of Mechanical Engineering Certified by..........

\section{Signature redacted ${ }^{\text {August 14, } 2015}$}

Accepted by

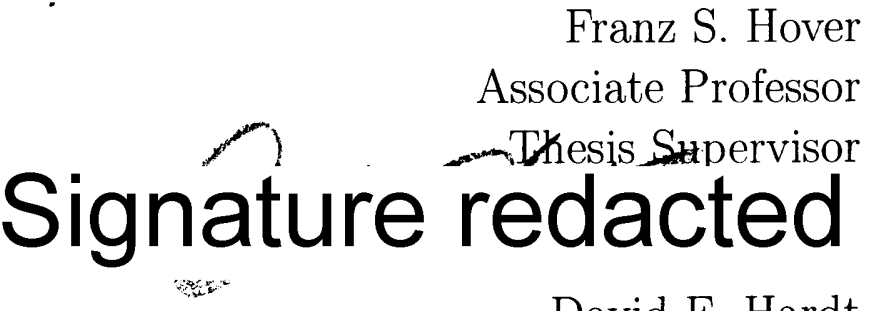

David E. Hardt

Graduate Officer, Department of Mechanical Engineering 


\title{
Navigation and Manipulation for Autonomous Underwater Dismantling of Offshore Structures
}

\author{
by \\ Christian Livingston Welch \\ Submitted to the Department of Mechanical Engineering \\ on August 14, 2015, in partial fulfillment of the \\ requirements for the degree of \\ Master of Science in Mechanical Engineering
}

\begin{abstract}
The dismantling of offshore structures, known as decommissioning, is a complex task in the oil and gas industry, driven by strict environmental standards. As the environments in which decommissioning is necessary become more challenging, autonomy presents itself as a solution to conduct this work as cost-effectively and safely as possible, namely by taking Remotely Operated Vehicles (ROVs) and divers out of the equation. In this thesis, three avenues are researched in support of Autonomous Underwater Vehicles (AUVs) for decommissioning: navigation, attachment, and manipulation. First, the Iterative Closest Point method (ICP) is investigated as a means to correct position drift of an inertial navigation system, by using a previously obtained coarse map. Using real sonar data from a current decommissioning site in the Gulf of Mexico, the algorithm is able to reconcile the internally dead-reckoned pose of the vehicle with that calculated via ICP, to an accuracy of $7 \mathrm{~cm}$ from a $100 \mathrm{k}-$ point sonar scan. Second, to attach lifting points to subsea scrap without bracing onto it, a single mechanism was designed to both drill and affix anchors in a single penetration, from a vehicle in free flight. A prototype was fabricated and its functionality verified. Third, to promote robust and stable robotic interactions using an industry standard non-backdrivable manipulator, a control law was developed to have the vehicle-manipulator system passively interact with its environment, by mimicking an arrangement of masses, springs, and dashpots. This control law was tested and analyzed in a simple experiment that achieved a $90 \%$ reduction in settling time.
\end{abstract}

Thesis Supervisor: Franz S. Hover

Title: Associate Professor 


\section{Acknowledgments}

The author would like to thank his colleagues and mentors at Chevron Energy Technology Company, who have provided valuable, first-hand experience in industry. $\mathrm{He}$ would also like to thank his labmates Pedro, Lucille, Brooks, Mei, and Josh, who have always given a helping hand, even at the slightest need. He would like to thank Daniel for the constant reminder that everything will be alright, and Ray for always making that possible in more ways than imaginable. Finally, the author would like to thank Prof. Franz Hover for having gone above and beyond his role as an advisor from the start, and without whom none of this would have been possible. 


\section{Contents}

1 Introduction $\quad 13$

2 3D Environment Mapping for Autonomous Navigation Around Sub$\begin{array}{lr}\text { sea Structures } & 19\end{array}$

2.1 Common Methods for Offshore Navigation . . . . . . . . . . . 20

2.1.1 Global Position System (GPS) . . . . . . . . . . . 20

2.1.2 Inertial Navigation System (INS) . . . . . . . . . . . 21

2.1.3 Doppler Velocity Log (DVL) . . . . . . . . . . . . . 21

2.1.4 Ultra Short Baseline (USBL) and Long Baseline (LBL) Acoustics 22

2.2 Navigation Complications with Respect to Autonomous Decommis-

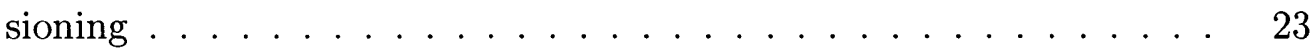

2.3 Using Iterative Closest Point (ICP) Method to Correct INS Integration

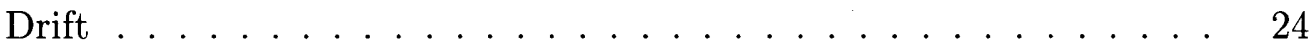

2.3 .1 Point Selection . . . . . . . . . . . . 27

2.3.2 Nearest Neighbor Determination . . . . . . . . . . . . . 29

2.3.3 Distance Minimization . . . . . . . . . . . . . . . . 29

2.4 Implementing ICP into an AUV Navigation Architecture . . . . . . . 33

2.5 Conclusions . . . . . . . . . . . . . . . . 36

3 AUV Bracing onto a Subsea Structure with Complex Geometry 39

3.1 Specific Challenges Faced in Subsea Bracing . . . . . . . . . 39

3.2 History of Contact Tasks from Free-Flight . . . . . . . . . . . . 41

$3.2 .1 \quad$ Space Docking . . . . . . . . . . . . . . 41 
3.2.2 Unmanned Aerial Vehicles (UAVs) . . . . . . . . . . . . 41

3.3 Virdrill: A Two-Stage, Reversible and Removable, Drilling and Tapping Mechanism . . . . . . . . . . . . . . . . 42

3.4 Prototyping for Component Functionality . . . . . . . . . . . . . . 42

3.4.1 Component Functionality . . . . . . . . . . . . . 45

3.4 .2 Drilling ............................ 48

3.4.3 Tapping \& Anchoring . . . . . . . . . . . . . . . . 49

3.4 .4 Removing .................... . . 50

3.5 Conclusions and Future Work . . . . . . . . . . . . . . . 50

\section{Autonomous Manipulation of Subsea Structures from a Free-Floating} $\begin{array}{ll}\text { Vehicle } & 53\end{array}$

4.1 Key Manipulation Tasks for Preliminary Phases of Decommissioning . 53

4.2 Using Passivity to Promote Robustness in Environment Interactions . 55

4.3 Experimental Setup . . . . . . . . . . . . . . 55

4.3 .1 Overall System Layout . . . . . . . . . . . . . . . 55

4.3.2 Hardware ....................... . . 58

4.3.3 Software and Communication . . . . . . . . . . . . 61

4.4 Analysis of Manipulator on Fixed-Base in Air . . . . . . . . . . . . . 62

4.4.1 Modeling the Linear Actuator . . . . . . . . . . . . . . . 64

4.4.2 Creating a Control Law for the Fixed-Base in-Air Case . . . . 71

4.4.3 Experimental Results . . . . . . . . . . . . . . . . 81

4.5 Analysis of the Vehicle-Manipulator System Free-Floating in Water . 84

4.5.1 Modeling the Raft and Linear Actuator . . . . . . . . . . . 84

4.5.2 Linearizing Thrusters . . . . . . . . . . . . . 86

4.5.3 Thruster Frequency Response . . . . . . . . . . . . . . . . . . 87

4.6 Conclusion . . . . . . . . . . . . . . . . . 89

5 Conclusions $\quad 93$

5.1 Summary of Work . . . . . . . . . . . . . . . . . . . . . 93

5.2 Future Work . . . . . . . . . . . . . . . . . 94 


\section{List of Figures}

1-1 Structure to be Decommissioned . . . . . . . . . . . . . 14

1-2 Simulated Loop Current in the Gulf of Mexico . . . . . . . . . . . . 15

1-3 Simulated Hurricane in the Gulf of Mexico . . . . . . . . . . . . . 16

2-1 Marlin AUV and Echoscope 3D Sonar Scanner . . . . . . . . . . 20

2 -2 USBL Diagram . . . . . . . . . . . . . . . . 23

2-3 Underwater Visualization Techniques . . . . . . . . . . . . 25

2-4 3D Sonar Scan of Structure . . . . . . . . . . . . . 26

$2-5$ ICP Failure Example . . . . . . . . . . . . . . . . . . 28

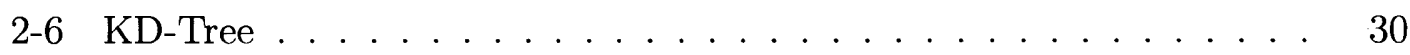

$2-7$ AUV Navigation Structure . . . . . . . . . . . . . . . 34

2-8 ICP Success Example . . . . . . . . . . . . . . . . 35

2-9 Principal Component Analysis . . . . . . . . . . . . . . 37

3-1 ROV plugging a hose into a service unit . . . . . . . . . . . 40

3-2 Virdrill Isometric and Profile Cutout . . . . . . . . . . . . . 43

3-3 AUV-ready Virdrill Isometric View . . . . . . . . . . . . . . . . . . . 44

3-4 Virdrill Key Bit . . . . . . . . . . . . . . . 45

3-5 Virdrill Drill Bit . . . . . . . . . . . . . . . 46

$3-6 \quad$ Virdrill Tap Sleeve . . . . . . . . . . . . . . . . . 47

3-7 Virdrill Anchor . . . . . . . . . . . . . . . . . . 48

3-8 Key Points in Drilling . . . . . . . . . . . . . . . . . 49

3-9 Key Points in Tapping . . . . . . . . . . . . . . . . . . 49

3-10 Key Points in Removing . . . . . . . . . . . . . . . . . 50 
$4-1 \quad$ Structure Biofouling . . . . . . . . . . . . . . . 54

$4-2 \quad$ Dexter ASV (1st Iteration) . . . . . . . . . . . . . . 56

$4-3$ Dexter ASV (2nd Iteration) . . . . . . . . . . . . . 57

$4-4$ Dexter ASV (Third Iteration) . . . . . . . . . . . 57

4-5 Linear Actuator Dissection . . . . . . . . . . . . . . . . . 58

4-6 Firgelli Automations Actuator and ATI Force/Torque Sensor . . . . . 59

4-7 Unimeasure String Potentiometer and RoboteQ Motor Controller . . 59

$4-8$ Inuktun Thrusters . . . . . . . . . . . . . . . 60

4-9 Dexter ASV Communication Architecture . . . . . . . . . . 61

4-10 RoboteQ Connection Diagram . . . . . . . . . . . . 62

4-11 RoboteQ Motor Control Utility . . . . . . . . . . . . 63

4-12 Dexter ASV Electronics Box . . . . . . . . . . . . . . . 64

4-13 Labview Project Block Diagram . . . . . . . . . . . . . . . 65

4-14 Model of Linear Actuator and Model of Ideal Mass-Spring-Dashpot System . . . . . . . . . . . . . . . . 6 65

4-15 Actuator Power-Speed Relationship . . . . . . . . . . . . . 69

4-16 Compensated Actuator Power-Speed Relationship . . . . . . . . . . 70

4-17 Linear Actuator Frequency Response . . . . . . . . . . . . . . . . . 72

$4-18$ Visualization of $|\operatorname{Arg}[h(j \omega)]| \ldots \ldots 73$

4-19 Passive Mass-Spring-Dashpot System . . . . . . . . . . . . . 73

4-20 Frequency Response of Ideal System With and Without Delay . . . . 76

4-21 Frequency Response of a Generic Lead Compensator . . . . . . . . . 77

4-22 Frequency Response of a Generic Lag Compensator . . . . . . . . . 78

4-23 Frequency Responses of Designed Lead-Lag Compensator and Individual Components . . . . . . . . . . . . . . . . 79

4-24 Frequency and Step Responses for Ideal, Uncompensated, and Compensated Systems . . . . . . . . . . . . . . . 80

4-25 Compliant-Tip Collision Comparison . . . . . . . . . . . . . 82

4-26 Force Input and Velocity Output Comparison . . . . . . . . . . 83

4-27 Dexter ASV System Model . . . . . . . . . . . . . . . . . . . . . 84 
4-28 Dexter ASV Ideal Passive Model . . . . . . . . . . . . . . . 85

4-29 Thruster Power-Force Relationship . . . . . . . . . . . . . . . 87

4-30 Linearized Thruster Power-Force Relationship . . . . . . . . . . . . . 88

4-31 Thruster Frequency Response . . . . . . . . . . . . . . . . . 90

4-32 Passive Raft-Manipulator Model . . . . . . . . . . . . . . . . . . . . 91 


\section{Chapter 1}

\section{Introduction}

The Gulf of Mexico has become a hotspot for offshore production in the oil and gas industry, with approximately 3,500 offshore facilities in the Gulf of Mexico [45], contributing $17 \%$ of the total crude oil production for the United States [22]. Dozens of these offshore structures in the Gulf of Mexico, like the one shown in Figure 1-1 reach the ends of their operational lives every year [9], and decommissioning, i.e., dismantling and removing, these structures in increasingly challenging environments has pushed the limits of Remotely Operated Vehicle (ROV) and diver capabilities. Decommissioning is often required for reasons such as: irreparable damage from natural events, degradation of structural integrity due to corrosion, or the lack of economic benefit to maintain production.

Natural events are a significant contributor to much of the damage sustained by offshore facilities in the Gulf of Mexico. Loop currents and hurricanes, two natural occurrences characteristic of this region, can impede, or even completely halt, an otherwise fully functioning offshore facility in a matter of days [20]. The Loop Current, unique to the Gulf of Mexico, shown in Figure 1-2, enters through the Yucatan Straits, loops into the Gulf of Mexico, and then exits through the Florida Straits. This loop current retreats in latitude at a frequency of every 4-16 months, and will shed a warm-core eddy typically between $150-450 \mathrm{~km}$ in diameter, which translates southeast at about $20 \mathrm{~km} /$ day on average [11] across hundreds of offshore facility sites. These eddies are notably warm and contribute to some of the fastest currents seen in the 


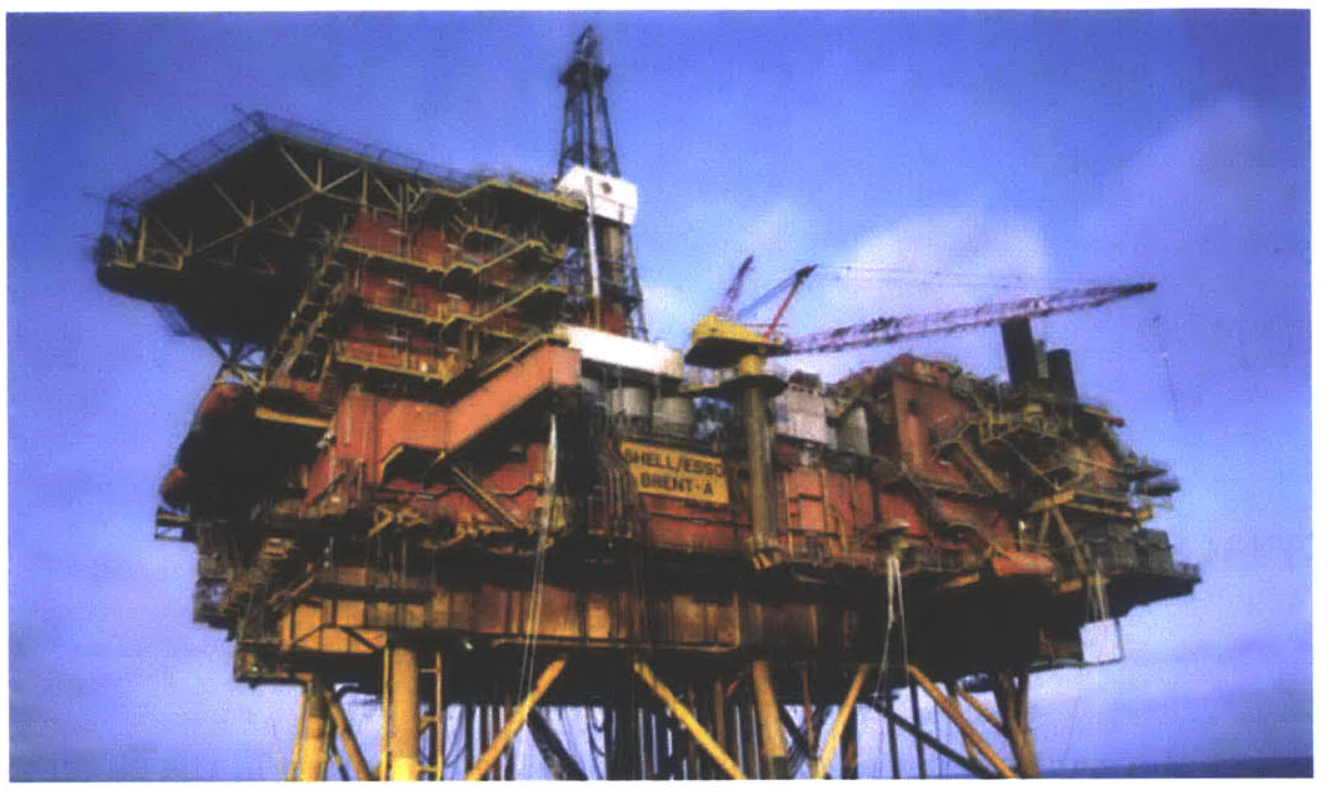

Figure 1-1: Topsides of a typical offshore structure in need of decommissioning [28].

Gulf of Mexico, even at depths of several hundred meters.

Hurricanes bring some of the same severe subsea conditions to offshore structures as the Loop Current, as shown in Figure 1-3, but also contribute wind conditions so hazardous that facilities in their paths must often be evacuated. A hurricane is a tropical cyclone that has a maximum sustained surface wind of at least $74 \mathrm{mph}$. In the Gulf of Mexico, they have the highest frequency of occurrence between June $1^{\text {st }}$ and November $30^{\text {th }}[26]$. In 2005 alone, hurricanes Katrina and Rita shut down $29 \%$ of U.S. refining capacity; 22,000 of 33,000 miles of pipelines, and 3.050 of 4.000 platforms were exposed to hurricane conditions, resulting in the destruction of 115 platforms, leaving 52 others damaged [31]. In 2008, hurricanes Gustav and Ike resulted in the loss of enough platforms to halt $1.05 \%$ and $1.3 \%$ of the daily production oil and natural gas in the Gulf of Mexico. The Gulf of Mexico sees around 100 tropical disturbances every year, and on average this results in 10 tropical storms and 5 hurricanes, bringing sustained winds of up to $175 \mathrm{mph}[33]$.

Hurricanes and the Loop Current play a large role in design criteria for offshore facilities in the Gulf of Mexico. The strength of these natural events influence mooring design and station-keeping, such as in determining the number and size of lines 


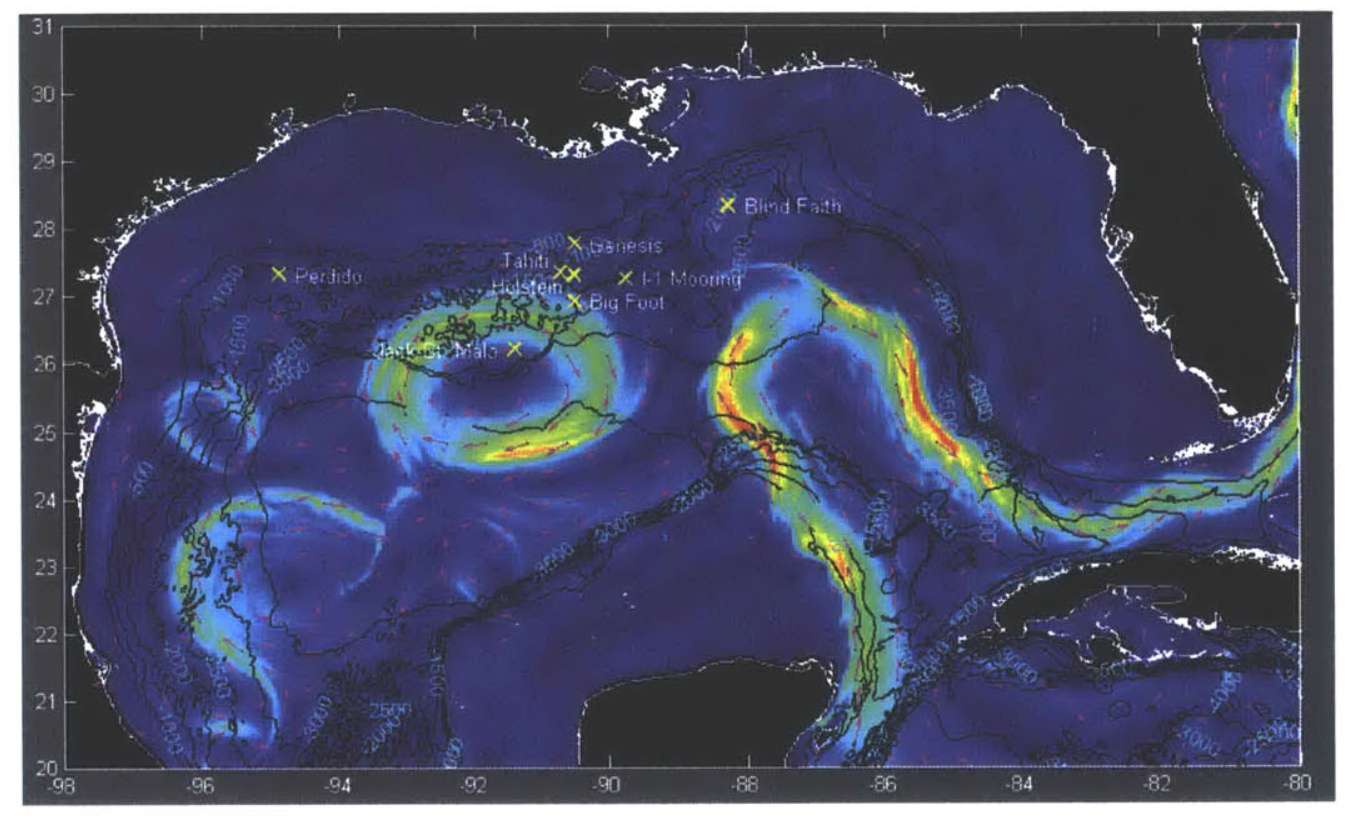

Figure 1-2: The Loop Current and an eddy exhibit sustained high surface current speeds in this color contour plot, generated using a 50-year free-run of the Hybrid Coordinate Ocean Model (HYCOM) developed by Florida State University in a Deepstar Joint Industry Project. Surface forcing conditions are provided by the Climate Forecast System Reanalysis (CFSR) record, ranging from 1992-2009, and are cycled three times for the 50-year model. The model boundary forcing conditions are derived from 4 years of a $1 / 12^{\circ}$ North Atlantic HYCOM simulation, and govern the inflow and outflow of the modeled Gulf Stream.

required for a floating facility, and determining the orientation of tethered Floating Production Storage and Offloading vessels (FPSOs) in tandem operations. The Loop Current is a particularly important factor in the decision to lower a riser from the platform, since these strong currents drive vortex-induced vibrations/motions (VIV and VIM), which contribute to fatigue damage in risers; additionally, excessive current load-induced curvature can inhibit drilling altogether [20].

Such environmental hazards put offshore structures at a high risk for damages that often require intervention, or even decommissioning. Much of this decision rests upon riser and structural integrity, since risers play an integral role in the oil production process, but are also one of the most risky assets associated with the platform. They are subject to fatigue loads without the assurance of redundancy in design, which means that any failure could be not only catastrophic, but also extremely expensive 

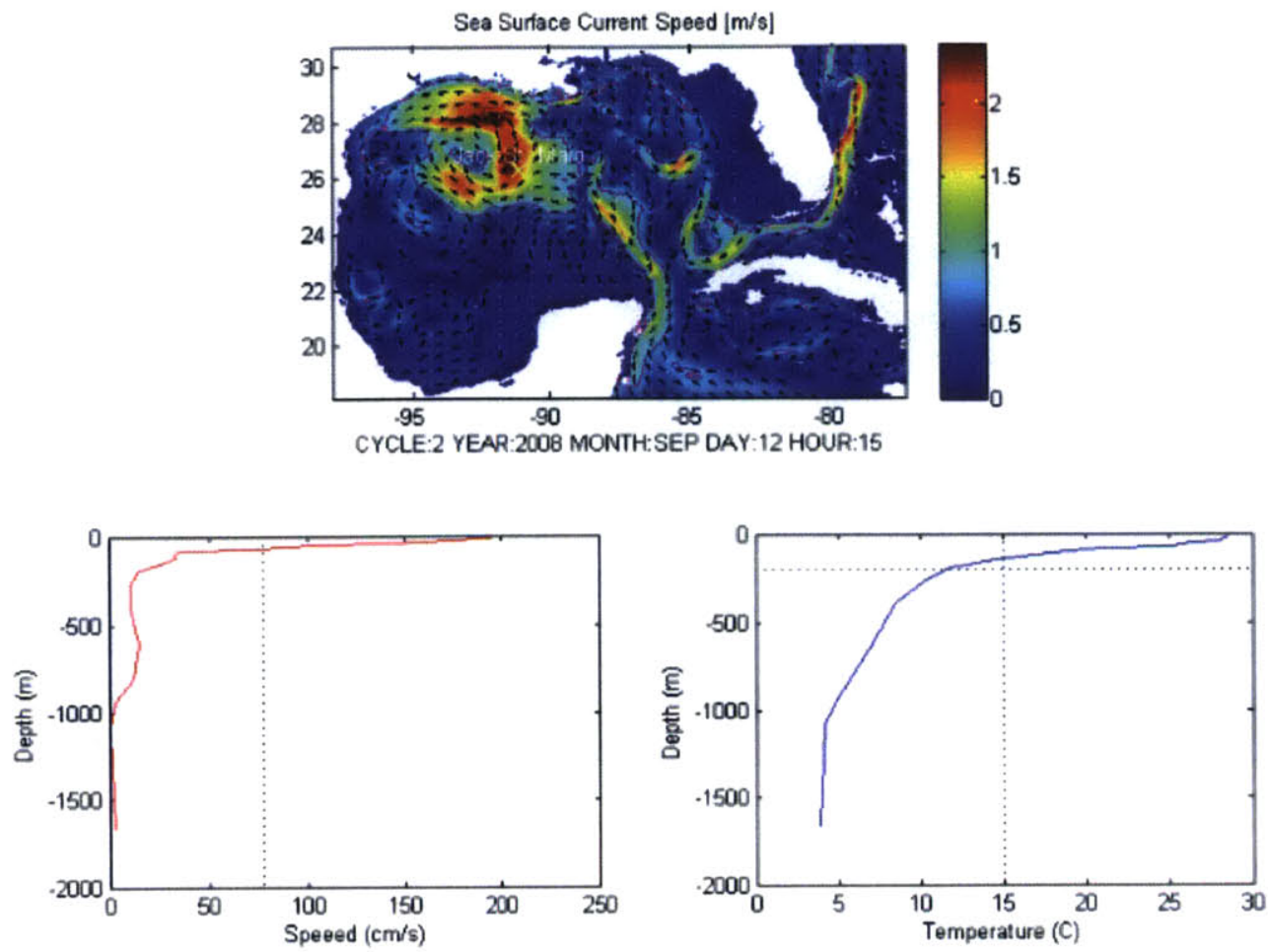

Figure 1-3: Simulated current profile from Florida State University HYCOM data shows a dramatic spike in sea surface speed, on par with Loop Currents, in the presence of a hurricane.

in terms of environmental remediation, physical repair, and downtime in production [10]. As previously mentioned, risers are often subjected to vortex-induced vibrations due to external disturbances caused by loop currents and hurricanes, which can shut down production for the duration of the natural event. Afterward, the riser must be thoroughly inspected, and repaired if necessary. However, if the structure itself has sustained damage due to catastrophic fatiguing, the facility may not be repairable, in which case it must be decommissioned.

\section{Outline of the Decommissioning}

Decommissioning is not only necessitated by natural events; it is an unavoidable end to most structures, due to either the finite production timeline of a well, or a structure not passing certification to produce past its planned operational life. A detailed process exists through which decommissioning is executed. According to 
Chevron's Environmental Management Company (Chevron EMC), decommissioning consists of ten specific phases [13]:

1. Planning and Engineering - CAD file and drawing searches, site visits for photo documentation, underwater inspection, pipeline studies

2. Well Abandonment - Disconnection from riser and permanent sealing of the wellhead

3. Conductor Removal - Lifting of conductors out of the water that were used to provide subsea power

4. Topside Preparation - Preparation of topsides to be removed from the rest of the structure

5. Subsea Preparation - Inspection of jackets to check for structural integrity to be lifted out of the water, determination of severing points, justification for potential reefing

6. Pipeline Abandonment - Flushing of pipelines, abandoned in place if covered, but must be lifted out of water if unburied or uncovered

7. Deck Removal - Heavy lift vessel is used to remove deck from the rest of the structure

8. Jack Removal - Lifting trunnions are attached and used to lift jacket from water

9. Disposal - Major pieces are taken to reef sites or scrapyards

10. Site Clearance: Trolling boats and ROVs are used to clear any additional debris left on site

Out of these ten phases of decommissioning, experts in Chevron EMC have explicitly stressed the importance of the very first phase of the decommissioning process - planning and engineering - as it determines the appropriate personnel, equipment, 
time, and money needed to successfully execute the subsequent steps [13]. Thorough underwater inspection is essential, given that many platforms are purchased from other companies without engineering drawings. Even for structures with adequate documentation, decades of severe environmental conditions could alter the subsea landscape in unforeseen ways.

Decommissioning today is both expensive and life threatening. The cost of ROV missions can cost on the order of hundreds of thousands of dollars per day, and when this rate is maintained over the course of a typical decommissioning time frame of several months, the costs become rather steep [13]. Additionally, by the nature of the work, there is no guarantee of having an ROV-friendly work environment, as unforeseen obstructions could potentially sever the tether. More importantly, near death experiences are not uncommon for commercial divers [19]. Severed oxygen umbilical cords [40] and a high pressure water jet injuries [41] are just a couple of many ways a routine commercial dive can go wrong. In some cases, such as arc-oxygen torch explosions [2], these accidents can be fatal.

As decommissioning environments become more severe over time, the industry is beginning to turn more towards autonomy to conduct these projects as cost-effectively and safely as possible, namely by removing ROVs and divers from the process. On one hand, AUVs must maintain the robust characteristics of their ROV counterparts. On the other hand, they must also demonstrate their ability to sense and manipulate their environment at least as well as the divers that are hoped to be replaced. Chapter 2 discusses a method to correct AUV navigation drift using real-time 3D sonar scans that achieves an accuracy of $7 \mathrm{~cm}$ from a $100 \mathrm{k}$-point sonar scan.. Chapter 3 introduces a mechanical design to attach lifting points into subsea scrap metal in a single penetration, for which a prototype was fabricated and its functionality verified. Chapter 4 investigates a passive control strategy for free-floating contact tasks using a non-backdrivable manipulator that results in a reduction in peak impact force and total settling time by $24 \%$ and $91 \%$, respectively. 


\section{Chapter 2}

\section{D Environment Mapping for Autonomous Navigation Around Subsea Structures}

During the Planning and Engineering phase of decommissioning, CAD files and engineering drawings are collected, accompanied by site visits to obtain photo documentation to compare against any pre-existing records found. Since many future decisions made for the project rely on discoveries found during the planning and engineering phase, it is important that initial surveying is done as accurately as possible the first. During these site visits, sometimes structures are not as they had been designed; this may be due to on-the-spot changes made during the fabrication process, sustained damage from natural events, or a number of other sources [13].

AUVs, such as Lockheed Martin's Marlin shown in Figure 2-1a, have been developed to help accurately document such deviations from pre-existing records. Using a side-scanning sonar, such as the Coda Echoscope pictured in Figure 2-1b, the vehicle can circle around the structure at a distance such that collisions with the structure's potentially complex geometry are avoided, and there is always a clear signal to the dynamically-positioned support vessel involved with the survey. The result of this process is a coarse, geographically oriented map of the structure, comprised of a collection of compiled $3 \mathrm{D}$ sonar scans. These $3 \mathrm{D}$ sonar survey maps could prove beneficial to 


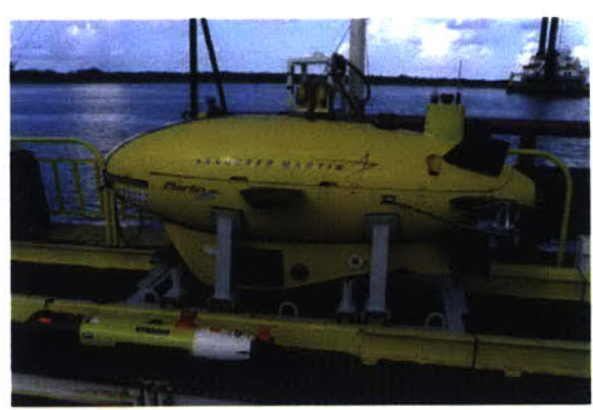

(a) Lockheed Martin's Marlin AUV [17]

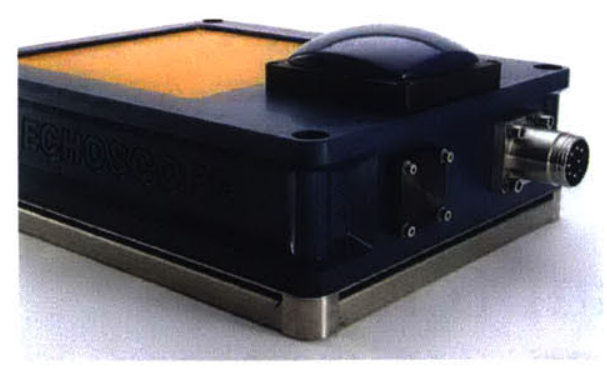

(b) Coda's Echoscope 3D Sonar Scanner [21].

Figure 2-1: Lockheed Martin's Marlin AUV and Coda's Echoscope 3D Sonar Scanner

navigation, where traditional navigation methods fall short in the face of the adverse conditions present in many decommissioning projects, which include poor visibility, dangerous jutting objects, and significant acoustic scatter. This chapter discusses the topic of subsea navigation for decommissioning in detail, delving into commonly used methods for offshore navigation by the oil and gas industry, their usefulness specific to decommissioning, and how subsea navigation can be greatly improved by incorporating 3D structure reference data.

\subsection{Common Methods for Offshore Navigation}

\subsubsection{Global Position System (GPS)}

Global Position System (GPS), a satellite based navigation system, is the most commonly used surface positioning system in existence, and is comprised of 24 satellites orbiting the earth. With four or more satellites within an unobstructed line of sight of a receiver, location and time information can be provided anywhere on earth, anytime of the day, and in any weather conditions. Position is calculated using trilateration via the differences in transmission time between the receiver and viewable satellites. A gyrocompass or magnetometer is often used in surface navigation to compensate for the lack of heading information using GPS. Gyrocompasses are often preferred over magnetometers since they find true north (as opposed to magnetic north) and 
do not experience interference from a metal hull and/or onboard electronics.

GPS signals do not carry underwater, and thus GPS has its usefulness in underwater navigation by keeping support surface vessels dynamically-positioned that aid in USBL navigation. Even onboard an AUV, GPS can be used for initial navigation calibration against an absolute measurement from the dynamically-position vessel prior to a dive, which would serve as a reliable starting point for inertial navigation system (INS)-based navigation.

\subsubsection{Inertial Navigation System (INS)}

An inertial navigation system is typically comprised of accelerometers and gyroscopes to measure linear accelerations and rotational velocities, respectively. From these acceleration readings, velocity, position, and orientation can continuously be calculated via integration of the sensor values, thus removing the necessity for external references. This is particularly useful in subsea applications in which communication is difficult, visibility is suboptimal, and GPS is unavailable.

INS errors are cumulative, since position and orientation are calculated by integration, and each subsequent calculation is based on the previous one - this is called integration drift. This implies that subsea navigation based solely on inertial measurements, also known as dead-reckoning, may not be suitable for long-term subsea missions without any correction measures in place. For this reason, other navigation aids that use external references, such as a Doppler Velocity Log, are used in conjunction with an INS.

\subsubsection{Doppler Velocity Log (DVL)}

A Doppler Velocity Log is an Acoustic Doppler Current Profiler adjusted to track sound waves bouncing off of the seafloor. By measuring the Doppler Effect induced from the moving vehicle, its velocity relative to the seafloor can be calculated, from which vehicle position can be derived. However, this estimation is highly sensitive to the velocity of sound [57], which is dictated not only by temperature, pressure 
and salinity, but also clutter in the work space. Therefore, pre-programming for local sound velocity would not be a trivial task. DVL errors are also heavily dependent on heading sensor precision and accuracy. Although past experiments [57] have shown a DVL-RLG (Ring-Laser Gyro) system yielding only a 0.25 -meter standard deviation over a 62-minute survey mission, this may not be appropriate for longer scale operations expected in decommissioning.

\subsubsection{Ultra Short Baseline (USBL) and Long Baseline (LBL) Acoustics}

Ultra short baseline (USBL) acoustics is a method for achieving subsea navigation, using a hydroacoustic transceiver and transponder. An acoustic pulse is sent from a vessel transceiver with known attitude, heading, and coordinates (typically derived from GPS), which then activates the transponder on an ROV to emit a response, illustrated in Figure 2-2. This response is then picked up by the vessel transceiver and used to find the transponder's range relative to the vessel. Bearing is determined from the differences in time of arrival between the multiple transceiver elements, similar to how human ears can infer the direction from which someone is shouting. Inverting this process could have useful applications for an AUV in which a transponder mounted on a dynamically positioned ship could provide global positioning for the vehicle conducting subsea operations. However, it should be noted that USBL error scales linearly with depth, and is therefore more useful in shallow water applications, where its accuracy is on the order of $1 \mathrm{~m}[12]$.

Long Baseline (LBL) acoustics work similarly to USBL, except that there are typically three or more transponders, which are fixed in a known position on the seafloor. Through this setup, the position of the transceiver can be triangulated in a similar fashion to GPS, with an accuracy that is much higher than what is typically achieved with USBL. Whereas USBL is easy to use but less accurate, LBL is time intensive due to array deployment, calibration, and recovery, but provides superior measurement readings an order of magnitude more accurate than USBL [12]. 

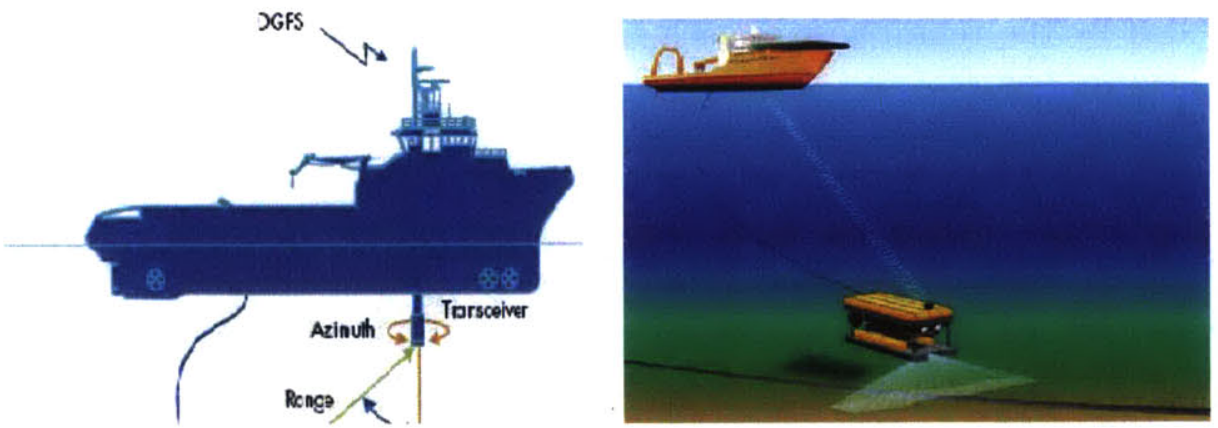

Figure 2-2: A dynamically positioned vessel uses its acoustic transceiver to infer the position of an ROV [12].

Acoustic-based navigation provides some of the best options for subsea navigation, due to its underwater functionality and avoidance of drift. However, with both USBL and LBL, accuracy will greatly be affected by acoustic performance around a cluttered environment, as what is to be expected during the task of decommissioning, which may result in both acoustic reflections and variable medium density through which acoustic signals would travel.

\subsection{Navigation Complications with Respect to Au- tonomous Decommissioning}

USBL and LBL are two forms of subsea navigation heavily used in industry today, but their effectiveness falls short in accomplishing the goal of decommissioning autonomously. Although using USBL with AUVs would help with ROV tether management, the costly necessity for a dynamically-positioned support vessel still exists. In the instance where a surface communication buoy is used in lieu of a support vessel, in a tethered scenario, the tether remains a risk in a cluttered environment. In an untethered case, the vehicle is at the mercy of both USBL's depth-limited range and acoustic reflections from the structure [12]. High costs are also associated with LBL given the specific application. LBL works best with a direct line-of-sight to the transponders [12]. Depending on the complexity of the structure, the number of transponders needed and the amount of acoustic reflections may rise rather quickly. 
Compounded by the rental cost of each transponder averaging out to thousands of dollars per day, and typical decommissioning projects lasting months at a time, the overall cost for LBL navigation becomes staggering [13].

Setting costs aside, USBL and LBL highlight the issue of accuracy when attempting to localize an AUV using sources outside of the immediate workspace, whether from a support vessel at the surface or acoustic transponders scattered away from the structure on the sea floor. This problem suggests the desirability of a navigation approach that does not rely heavily on external references, but inertia-based navigation drifts. However, there are several visualization-based aids that could be integrated into underwater navigation to provide a way of correcting this integration drift that plagues all INSs. Video is the most common, but debris from contact tasks is an is-

sue explicitly expressed by industry experts [13]. Although laser scanning has better range, the problem of turbidity still lingers since it is still optics-based. However, 3D sonar imaging is able to provide a long enough wavelength such that cloudiness in the water does not affect the visualization to nearly the same degree in local situations, resulting in less attenuation and longer range. Unlike other forms of sonar, 3D sonar imaging analyzes the strength of return signals from projected sound energy in order to detect objects [49].

\subsection{Using Iterative Closest Point (ICP) Method to Correct INS Integration Drift}

Given the unique challenges faced with subsea decommissioning tasks, acoustics-based techniques are a promising navigational aid. More specifically, 3D sonar imaging could be used as an external reference in order to correct integration drift in real time. As previously mentioned, one of the most important steps in the decommissioning process is structure surveying. It is not uncommon for the underwater inspection of an offshore structure to consist of using a vehicle equipped with a $3 \mathrm{D}$ sonar scanner, tethered to a support vessel from which it can obtain an absolute position reference. 


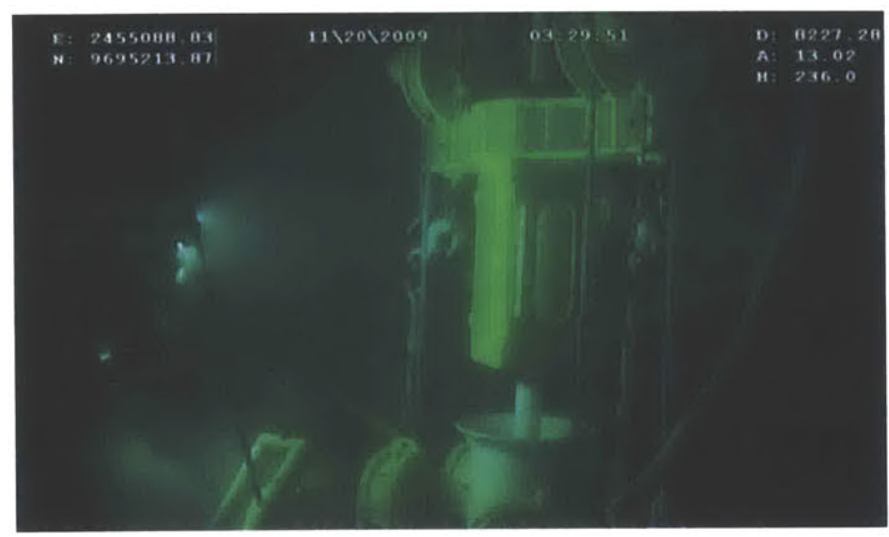

(a) ROV Video Footage

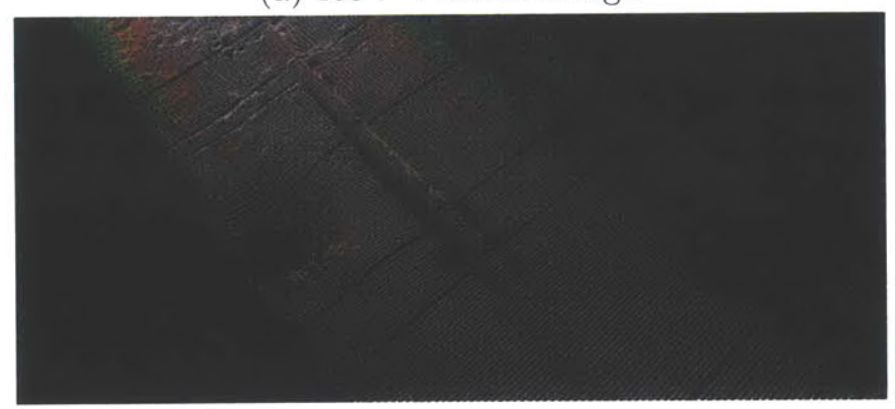

(b) Laser Scan

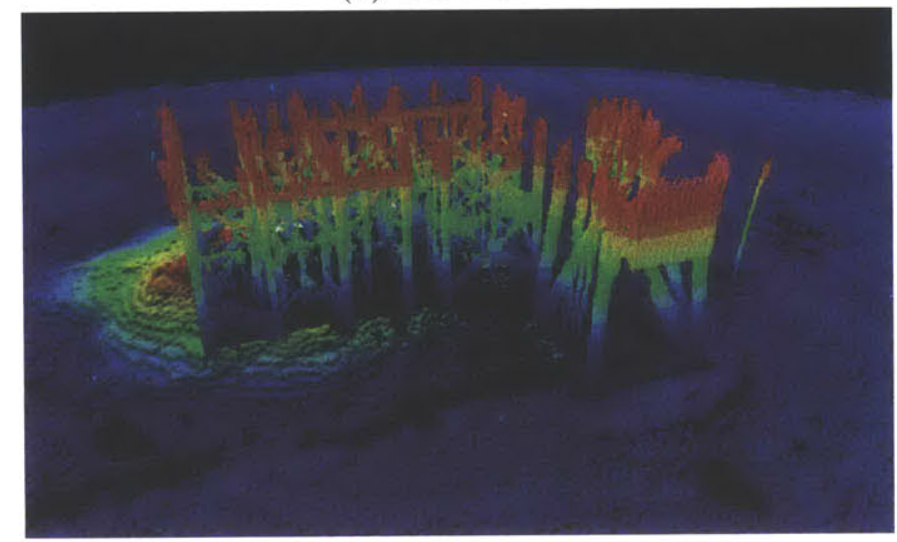

(c) Sonar Scan

Figure 2-3: Industry images of ROV video footage (2-3a) [14], laser scan (2-3b) [15], and acoustic scan (2-3c) [16]. Although video footage provides superb resolution, it is highly susceptible to turbid conditions, which are a common consequence of interaction with the structure. Laser scanning, which provides better range than video and higher resolution than acoustic scanning, has been valuable to pipeline inspection, but is still subject to the same turbidity issues faced with video. Although acoustics may not have the same resolution as optics, the range afforded in turbid waters makes acoustics an attractive imaging method for autonomous decommissioning. 
For this particular study, data was collected from Lockheed Martin's Marlin AUV that was used to take 3D sonar snapshots of a structure in the Gulf of Mexico in need of decommissioning. The product is a coarse 3D map of the structure with absolute position references and $\sim 5 \mathrm{~cm}$ position error [51]. The following section describes a way in which an AUV tasked with decommissioning a structure can correct its INS drift and orient itself by comparing the pose associated with its real-time sonar scans to that of its previously obtained 3D survey map, using the Iterative Closest Point (ICP) method.

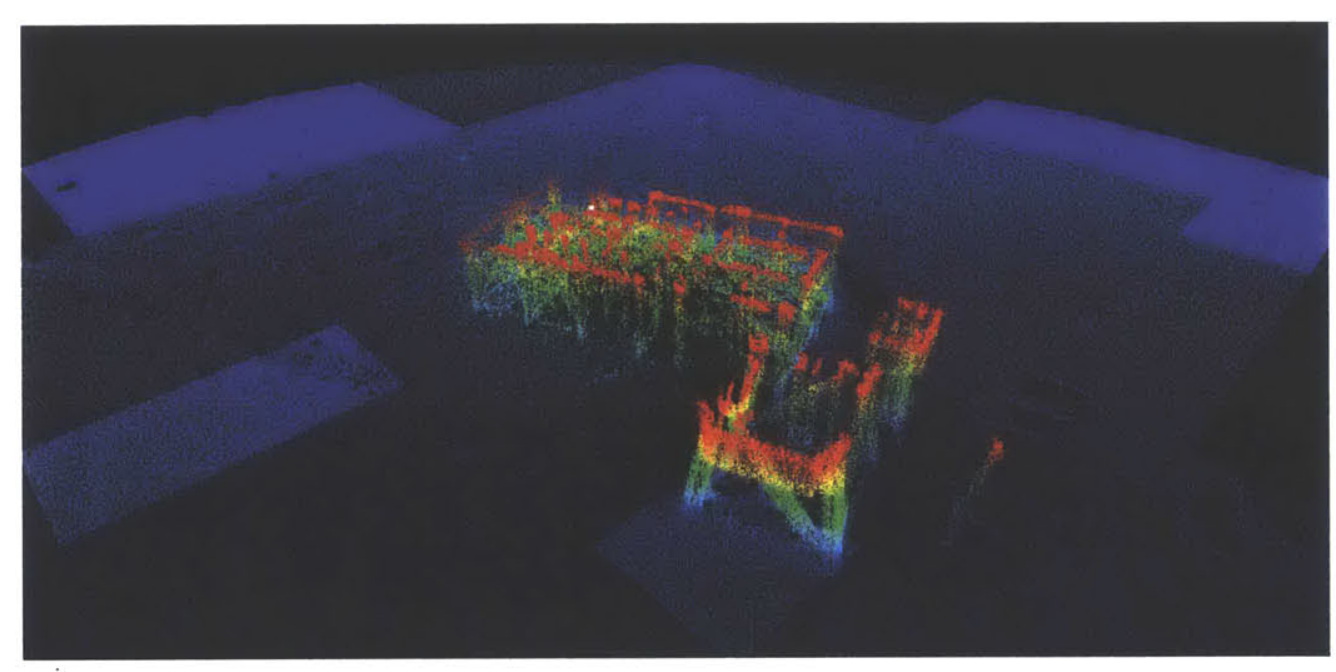

Figure 2-4: 3D Sonar data from the Marlin AUV visualized through Leica Cyclone. The structure pictured stands at approximately $11 \mathrm{~m}$.

Iterative Closest Point is a method originally developed for correcting scans in positron emission topography [5], but has shown its value in being repurposed for AUV localization. ICP has found its way in successes with vehicle localization in many other respects, such as 2D land-based navigation using laser scanners [43]. Closer analogs to potential AUV usage have been demonstrated, such as through the use of acoustics-based ICP in land-based navigation [13], however, this example uses sixteen separate sonar scanners, which may not necessarily be economically feasible in an AUV application. The research presented in this section takes concepts previously introduced in other navigation scenarios, and evaluates its feasibility specifically in an underwater environment where only one sonar scanner is available, 
using 3D sonar imaging data of decommissioned structures, provided by Lockheed Martin. An algorithm implementing ICP takes a sonar scan and a reference map, and uses least-squares minimization to calculate the necessary rotation and translation needed to make the two data sets align as perfectly as possible. The Iterative Closest Point method consists of three major steps - point selection, nearest neighbor determination, and distance minimization.

\subsubsection{Point Selection}

During the first step of point selection, the algorithm determines what portion of the pre-loaded 3D reference map to use for the correction. Given that it is desired to have this algorithm run in real time, it would be naïve to feed the algorithm the entire pre-loaded map regardless of scan size or domain, especially given that with a typical full map size being greater than 14 million points [38], there is a risk for not only slow computation time, but also getting stuck in local minima in a random and irrelevant portion of the map, shown in Figure 2-5.

However, although ICP is being used to correct INS drift, the INS provides the best available guess of the vehicle's true location, and this information could be used to perform an INS-assisted conservative reduction of the reference map. Specifically, if a bounding box is created to reduce the entire map to the regions of the map where the vehicle could have reasonably traversed up to the point of correction, the

irrelevant portions of the map can be rejected from the scan matching. Implementing such a process demonstrated a reduction of computational time up to $120 \mathrm{~ms}$.

Given that ICP is running continuously to prevent position error from growing, this implementation also showed a less likely convergence on an irrelevant local minimum, which is especially important since convergence on a local minimum, as opposed to the global minimum, is one of the greater problems with ICP [34]. Continuously running the algorithm ensures that the bounding box remains small in size, and the initial guess of position before running ICP is not wildly off. Otherwise, a dense, unbounded point cloud reference map could lead to faulty nearest neighbor determination, resulting in a possible solution at a number of undesirable local minima, thus 


\section{Iterative Closest Point Method for AUV Localization}
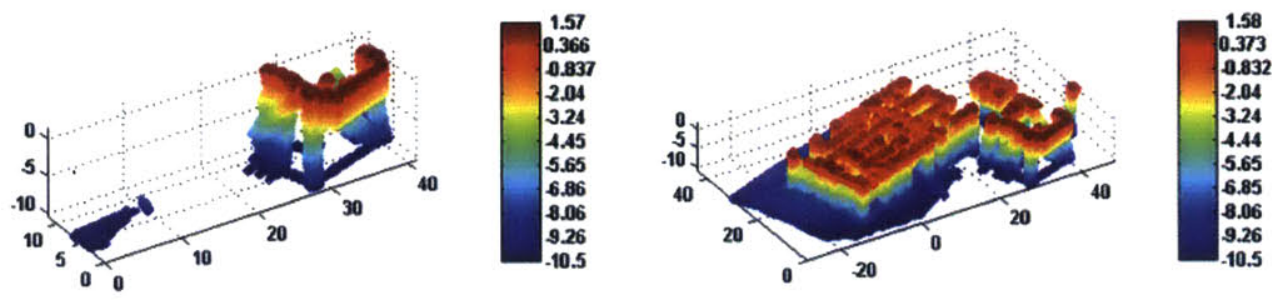

Red: Original Data | Blue: Transformed Point Cloud
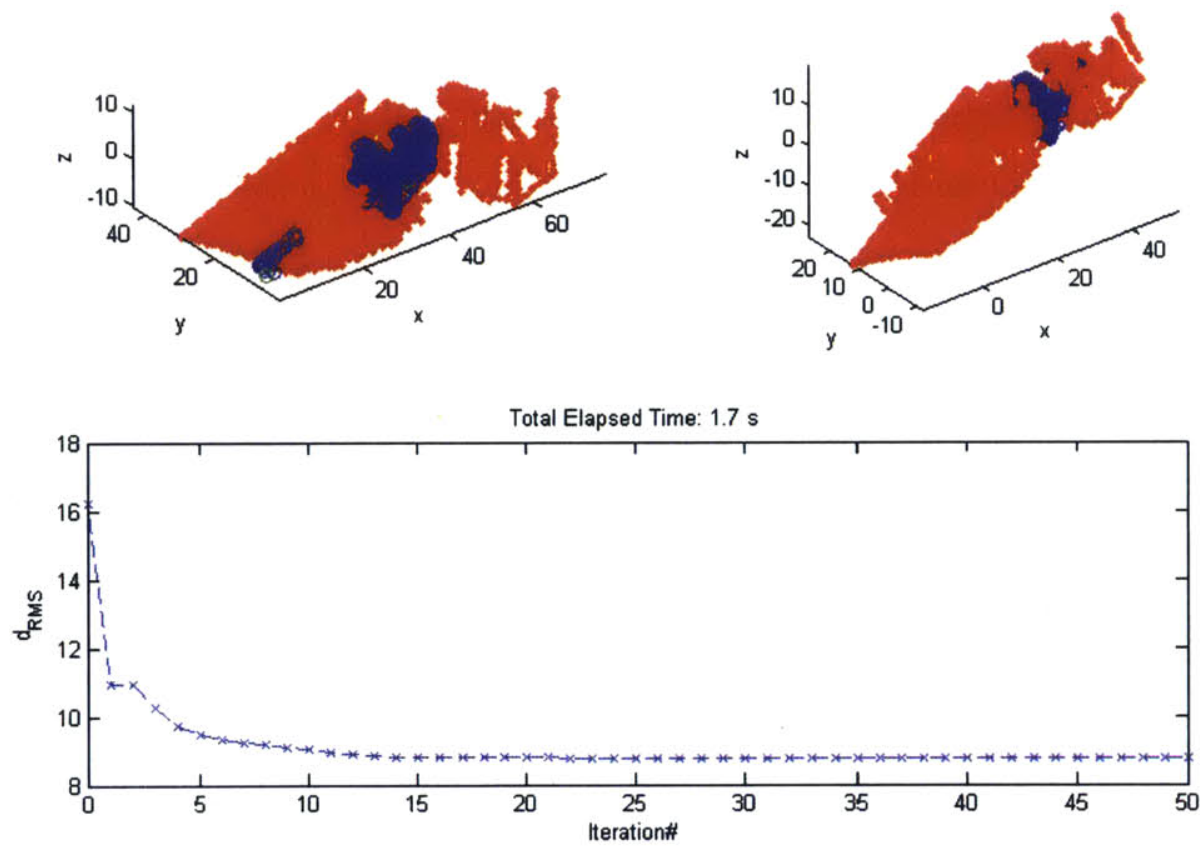

Figure 2-5: The top-left image shows an AUV sonar scan, whereas the top-right scan shows the entire stored reference map. The middle-left panel shows where an AUV sonar scan (blue) places on a reference map (red) prior to ICP correction. The middleright panel shows the ICP result, which does not indicate a proper matching onto the structure. A local minimum, as opposed to the absolute minimum, is reached, which can be avoided using point selection techniques. The bottom plot shows characteristics of a converged solution, however, the RMS converges to an unacceptably high value. All units are in meters. 
making convergence on the global minimum extremely difficult.

\subsubsection{Nearest Neighbor Determination}

The second step in the ICP method deals with pairing nearest neighbors between the reduced selection of points from the reference map to the current 3D sonar scan. When completing this step, the task at hand is to find the most efficient way to find the nearest neighbor point $q$ in the reduced reference map to each point $p$ in the current $3 \mathrm{D}$ sonar scan.

The naïve approach to do this would be to exhaustively compare each point in the scan to every single point in the reference map, which means that if a single scan has, for example, a thousand points and the reference map also has a thousand points, there would be a million computations per scan.

Alternatively, a KD-tree is a way to structure the reference map data for quick and efficient searching. The organizational approach works by dividing the point cloud reference map into $x$-wise, then $y$-wise, then $z$-wise median divisions, in succession until all points have been compartmentalized. The median point for the first split is the root, and the last possible split creates the leaves. In Figure 2-6, the red box denotes an $x$-axis split. If the point to be paired to its nearest neighbor is to the left of that split, all the points to the right of it can be thrown away. This process can be continued for each dimension until a nearest neighbor is found.

A larger the data set corresponds to a relatively more efficient KD-tree. For example, if a reference map has a thousand points, to match a point in a scan takes $\log _{2}(1000)$ points, which is approximately 10 calculations, as opposed to 1000 taking the brute force approach [8].

\subsubsection{Distance Minimization}

In the previous two steps, a subset of points were selected from the reference map, and then were paired to their nearest neighbors. In the third step of the scan matching process, point-to-point minimization, the task at hand is to find the optimal amount of 


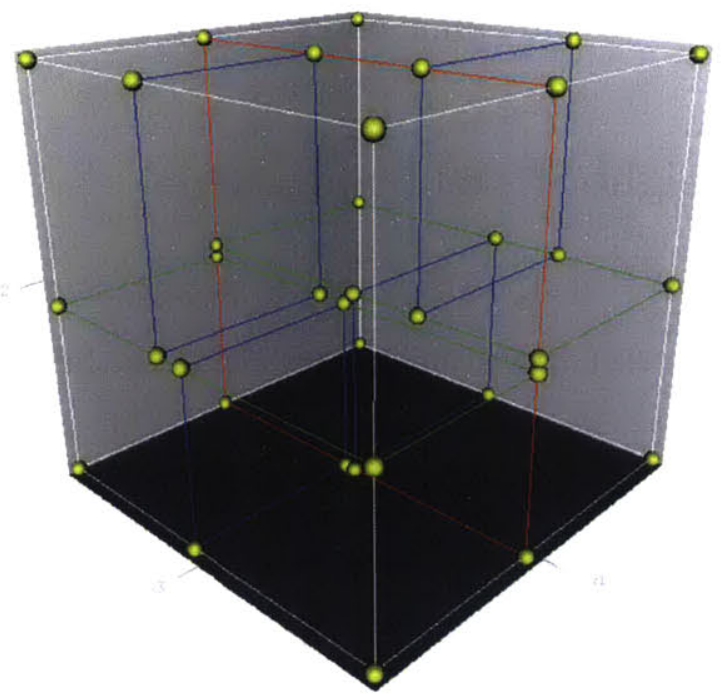

Figure 2-6: Visualization of how data points are divided using the KD-tree: the red box indicates the first division of the data set. If the point of interest is to the left of the red box, all points to the right may be discarded [7].

rotation and translation of the current 3D sonar scan to minimize the total Euclidean distance between each of the point pairs with the reference map.

Linear algebra provides a method to find an answer using Singular Value Decomposition (SVD) [5], through which a matrix transformation $M$ is decomposed into a rotation matrix $U$, a non-negative real-numbered rectangular scaling matrix $\Sigma$, and another rotation $V$. Once again, given points $q$ in the reduced reference map and points $p$ in the current 3D sonar scan, the closed form solution to minimize error in Euclidean distance is:

$$
\min \left(\sqrt{\left(q_{1}-p_{1}\right)^{2}+\left(q_{2}-p_{2}\right)^{2}+\ldots+\left(q_{n}-p_{n}\right)^{2}}\right)
$$

The variables considered are: the current $3 \mathrm{D}$ sonar scan centroid, $\bar{p}$, the centroid of the reference map subselection, $\bar{q}$, current scan deviations from the centroid, $p_{i}^{\prime}=p_{i}-\bar{p}$, reference map deviations from the centroid, $q_{i}^{\prime}=q_{i}-\bar{q}$, the rotation correction matrix, $R$, and the translation correction vector, $T$.

In order to minimize the total Euclidean distance, the following error equation 
must be minimized [34]:

$$
E=\sum_{i=1}^{N}\left\|R p_{i}^{\prime}+\vec{T}-q_{i}^{\prime}\right\|^{2}
$$

This error function is defined by the sum of the squared distances between each nearest neighbor pair between the current 3D sonar scan and reference map, after the rotation correction matrix and translation correction vector have been applied. This error equation can be expanded and simplified to produce the following equation:

$$
E=\sum_{i=1}^{N}\left\|p_{i}^{\prime}\right\|^{2}-2 \operatorname{tr}\left(R \sum_{i=1}^{N} p_{i}^{\prime} q_{i}^{\prime T}\right)+\sum_{i=1}^{N}\left\|q_{i}^{\prime}\right\|^{2}
$$

Define the inner product as:

$$
N=\sum_{i=1}^{N} p_{i}^{\prime} q_{i}^{\prime T}
$$

Substituting $N$ in to the error equation produces the equation below. From here, it can be seen that in order to minimize the error in total Euclidian distance between all point pairs, the trace of the inner product, $N$, multiplied by the rotation correction matrix, $R$, must be maximized.

$$
E=\sum_{i=1}^{N}\left\|p_{i}^{\prime}\right\|^{2}-2 \operatorname{tr}(R N)+\sum_{i=1}^{N}\left\|q_{i}^{\prime}\right\|^{2}
$$

The singular value decomposition of the covariance matrix $N$ of the two point clouds is used in order to solve for the optimal rotation, $R$, in the error minimization function, whose solution can be found as follows.

Assume that the rotation matrix takes the form:

$$
R=A B^{T}
$$

Factor $N$ using singular value decomposition, remembering that a matrix transformation can be decomposed into a rotation $U$, a scaling $\Sigma$, and another rotation $V$ this is a square matrix:

$$
N=U \Sigma V^{T}
$$


Plug both $R$ and $N$ into the trace function that must be maximized:

$$
\operatorname{tr}(R N)=\operatorname{tr}\left(A B^{T} U \Sigma V^{T}\right)
$$

Given that $R$ is a rotation matrix, the maximum trace achievable is upper-bounded by the following equation, where the trace of $N$ is the sum of the dot product of the $p-q$ point cloud pairs:

$$
\operatorname{tr}(R N) \leq \operatorname{tr}\left(\sqrt{N^{T} N}\right)
$$

Apply SVD to $\operatorname{tr}\left(\sqrt{N^{T} N}\right)$ :

$$
\operatorname{tr}\left(\sqrt{N^{T} N}\right)=\operatorname{tr}\left(\sqrt{V \Sigma^{T} U^{T} U \Sigma V^{T}}\right)
$$

Because rotation matrices are unitary, meaning that the transpose of a rotation matrix multiplied by itself is the identity matrix $\left(U^{T} U=I\right)$, and they are also orthogonal, meaning that the transpose of a rotation matrix is equal to its inverse $\left(V^{T}=V^{-1}\right)$, the singular value decomposition of $\operatorname{tr}\left(\sqrt{N^{T} N}\right)$ can be simplified to the following:

$$
\operatorname{tr}\left(\sqrt{V \Sigma^{T} U^{T} U \Sigma V^{T}}\right)=\operatorname{tr}\left(\sqrt{V \Sigma^{T} \Sigma V^{-1}}\right)
$$

Additionally, since the scaling matrix is non-negative and diagonal, the following simplification can be made:

$$
\begin{gathered}
\operatorname{tr}\left(\sqrt{V \Sigma^{T} \Sigma V^{-1}}\right)=\operatorname{tr}\left(V \Sigma V^{-1}\right) \\
\therefore \operatorname{tr}\left(\sqrt{N^{T} N}\right)=\operatorname{tr}\left(V \Sigma V^{-1}\right)
\end{gathered}
$$

Therefore, from above, it can be seen that $R \equiv A B^{T}=V U^{T}$ in order to obtain the largest possible trace, i.e. the smallest possible error:

$\operatorname{tr}(R N)=\operatorname{tr}\left(A B^{T} U \Sigma V^{T}\right)=\operatorname{tr}\left(V U^{T} U \Sigma V^{T}\right)=\operatorname{tr}\left(V \Sigma V^{T}\right)=\operatorname{tr}\left(V \Sigma V^{-1}\right)=\operatorname{tr}\left(\sqrt{N^{T} N}\right)$

In other words, the optimal rotation correction matrix is found by taking the two 
rotational components of the singular value decomposition of the inner product of the nearest neighbor pairs between the reference map and the current scan:

$$
R=V U^{T}
$$

The translation correction vector is found by simply taking the difference between the centroids of the reference map, $\bar{q}$, and that of the rotationally corrected current scan, $\bar{p}$ :

$$
\vec{T}=\bar{q}-R \bar{p}
$$

\subsection{Implementing ICP into an AUV Navigation Ar- chitecture}

Overall, the navigation control loop can be described as follows: the vehicle generates a scan of the environment, which is then matched to the reference map of the structure using ICP in the three steps previously explained. That correction is then applied to the INS to reorient the vehicle, and then its path is corrected to account for the drift accumulated during the journey. This process is repeated continually throughout the AUV mission, which is visually represented in Figure 2-7.

As long as the real-time sonar scanning of the structure is able to capture features distinct enough to enable a convergence in the ICP algorithm, time-dependent integration drift is reduced, as in Figure 2-8. Many works have pointed to effective variants based on the ICP framework in order to improve the rate of convergence, such as the mechanical scanning imaging sonar probabilistic iterative correspondence method (MSISpIC) [29], metric-based iterative closest point method (mbICP) [42], and other high-speed variants [25], which should be integrated into a more comprehensive implementation of the navigation architecture. Given that many inertial measurement units can still maintain centimeter resolution over the course of a few seconds [58], the results from using the ICP method should not be hindered from a computational time of less than 0.2 seconds as shown in Figure 2-8. Instead, the 
AUV Navigation Structure for 3D Sonar-Aided

Localization in Robotic Autonomous Decommissioning

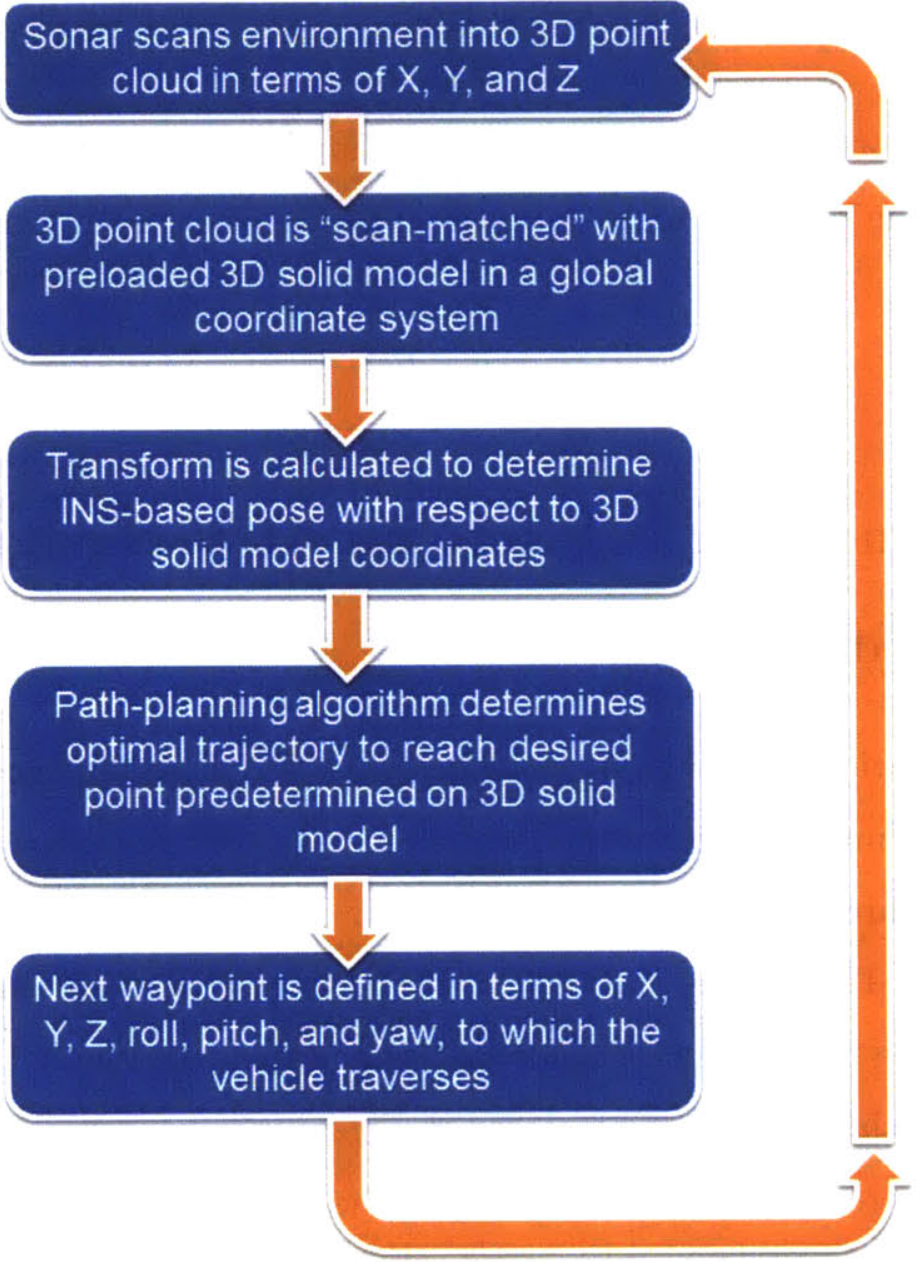

Figure 2-7: A general AUV navigation structure, augmented to include scan-matching INS correction.

limiting factor is then defined by the noise contributed by both the original 3D sonar reference map and the real-time sonar scans of the decommissioning vehicle.

Lockheed Martin states that their Marlin is capable of producing maps with a position error of $5 \mathrm{~cm}$ [51], but for any decommissioning tasks requiring position error less than $5 \mathrm{~cm}$, multiple forms of visualization can be incorporated into the navigation system. Namely, switching to laser scanning from sonar scanning in very close 


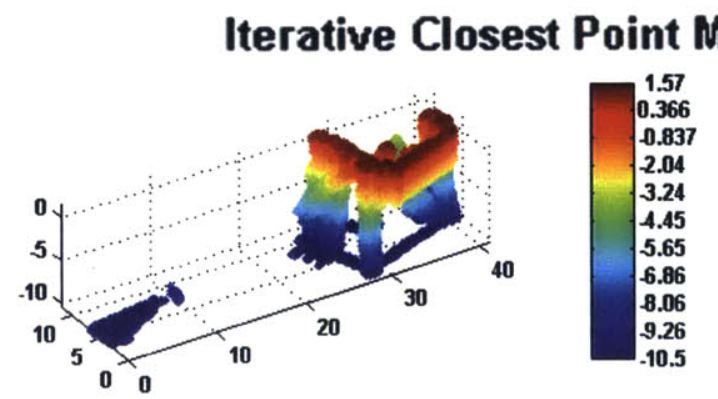

Red: Original Data | Blue: Transformed Point Cloud
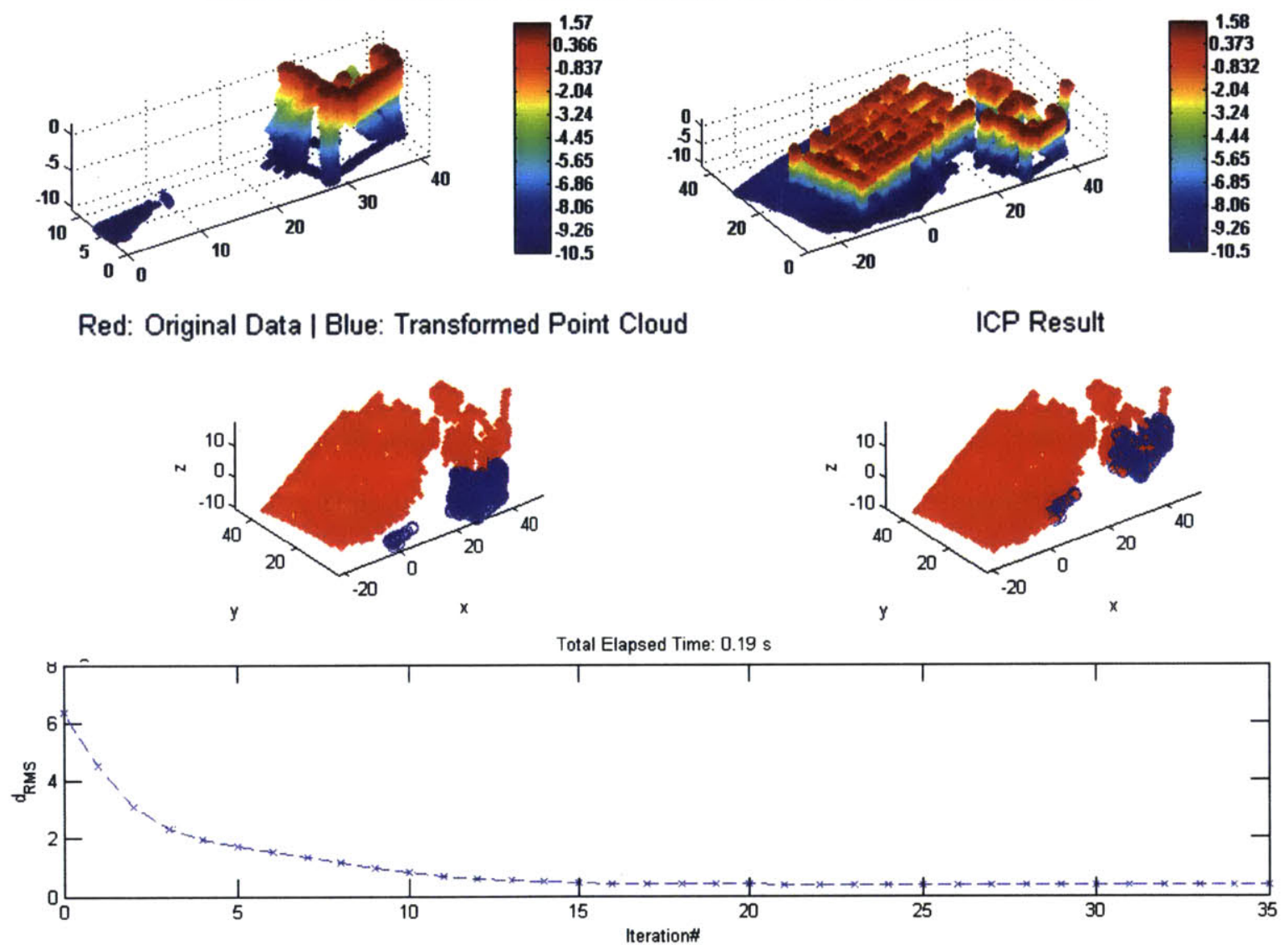

Figure 2-8: Trial of ICP scan-matching algorithm shows a convergence over 35 iterations in less than 0.2 seconds of an AUV sonar scan (top-left) against a reference map (top-right). The middle-left panel shows where the same AUV sonar scan (blue) places on the reference map (red) prior to ICP correction. The middle right panel shows the ICP result, which indicates a proper matching onto the structure, supported by a near-zero RMS convergence value on the order of centimeters. Due to various sources of noise in both the reference map and the AUV scan, it is impossible to achieve an RMS convergence value of zero. All units are in meters. 
proximity to the point of interest can provide accuracies of less than in inch [55], thus serving as a complement to the robustness of sonar scanning navigation in the longer range applications.

\subsection{Conclusions}

Although there is promise in the current implementation of navigation correction using Iterative Closest Point method, there are still a number of avenues through which substantial improvements could be made. Future work on $\mathrm{C}++$ implementation could greatly improved the runtime for the navigation routine, but could also provide versatility in implementation across various platforms. Specific to the distance minimization step of the ICP method, another approach, point-to-plane minimization, could be used as opposed to point-to-point minimization [8]. This method may be especially useful for a more accurate representation of a matched scan, since no two points are ever a perfect match, and reduce the calculated RMS convergence value. By defining the $1^{\text {st }}$ and $2^{\text {nd }}$ principal components as the two axes with most variation, those two axes define a plane, from which the $3^{\text {rd }}$ principal component as defined as the surface normal, shown in Figure 2-9. Point-to-plane minimization uses Principal Component Analysis to define surface normals for each point in the reference map, from which distances from point $p$ to the plane generated at point $q$ are measured. Taking such an approach could provide a potential solution to inconsistent point-density issues between real-time scan data and a pre-obtained reference map. Additionally, although the pre-obtained reference data may be filtered for noise, it may prove to be beneficial to incorporate an alternative definition of distance between two points of interest; namely, instead of using Euclidian distance, Mahalanobis distance can be utilized, which incorporates a random distribution of noise in data when calculating the error metric [43].

Decommissioning is a task that requires the ability to see a structure well enough to interact with it in a safe and effective manner. Although the current state of practice has pulled this off successfully, it has done so with the incorporation of a 


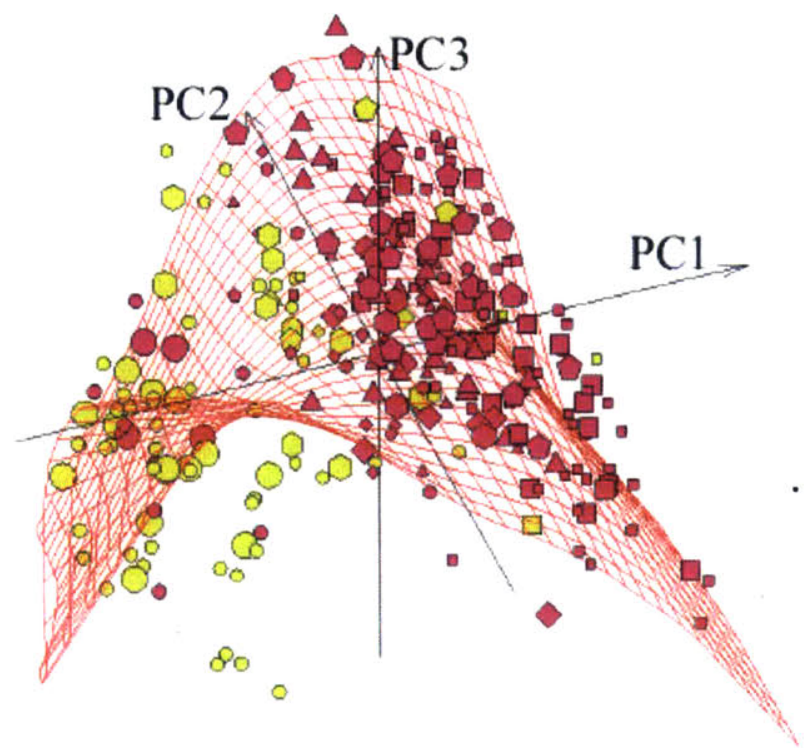

Figure 2-9: This visualization of principal component analysis shows the creation of a mesh using calculated surface normals [27].

real-time human-in-the-loop, whether it be from an ROV video feed, or the eyes of a commercial diver. However, in the case where the decommissioning task is done autonomously, a method must be in place to assure that at least a comparable level of navigation can be accomplished. By incorporating 3D sonar scan matching into an AUV's navigation system, a vehicle equipped with a $3 \mathrm{D}$ sonar scanner can mitigate the effects of integration drift, thus allowing for consistent, centimeter-level accuracies over its entire flight duration. 


\section{Chapter 3}

\section{AUV Bracing onto a Subsea}

\section{Structure with Complex Geometry}

Provided that an AUV is able to successfully and accurately navigate to a site of interest, the next challenge is initiating contact with a structure from free flight. There are a number of reasons for such an interaction, including the need to perform contactbased tests on a structure, such as removing biofouling from complex geometries to perform non-destructive testing (NDT) [25], or evaluating the functionality of valves and levers. A particular criterion set forth by Chevron EMC indicated the need to attach an anchor, or a set of several anchors, onto severed sections of the steel structure to be decommissioned. These would then need to be lifted from the seafloor and onto a floating barge. What makes this task particularly complex for AUVs is that simple gripping mechanisms found on common underwater manipulators are much less dexterous than divers. However, by solving this anchoring problem, a solution to bracing is simultaneously found, which can be applied to a number of other subsea tasks where free-flight interactions would otherwise be too difficult.

\subsection{Specific Challenges Faced in Subsea Bracing}

The particular task of affixing an anchoring point to a piece of subsea scrap consists of three key mechanical steps: drilling, tapping, and attaching. These encompass the 
creation of a hole, which would then need to be threaded, and then used to securely screw in an attachment point. The particular difficulty involved with performing these tasks independently comes from having to overcome a peg-in-hole problem from a free-flight vehicle, i.e., placing a tool into a hole in the workpiece with a tight clearance. ROVs are currently able to accomplish peg-in-hole tasks, however this ability is strongly dependent on the skillset of the particular ROV operator. Even in cases where partial ROV bracing is available, currents and vehicle dynamics can make tasks such as remote-controlled plug insertion unnecessarily time-consuming and extremely difficult [6], and can add up to thousands of dollars over time [47].

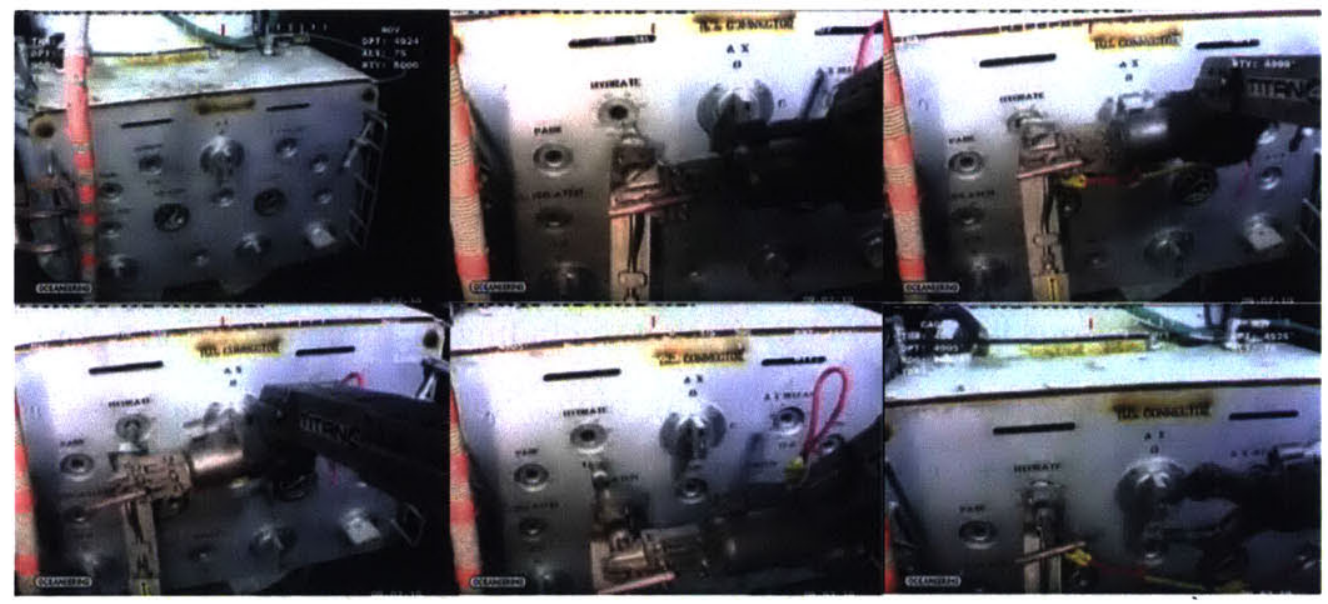

Figure 3-1: A working class ROV spends ten minutes plugging a hose into a service unit after missing the grabbing point, missing the insertion hole, and jamming the hose multiple times before a successful mating [6].

On the side of autonomy, the current state of experimental AUVs for intervention has demonstrated autonomous plugging/unplugging. However, these tasks have only been performed while docked to a subsea panel, which avoids the free-flight challenge presented in many decommissioning environments where preexisting vehicle docking infrastructure is not readily available [46]. In this chapter, a mechanical design is posed as a solution for simplifying the anchoring process into a single mechanism that eliminates the peg-in-hole problem completely, and can facilitate both ROV pilot and autonomous system performance. 


\subsection{History of Contact Tasks from Free-Flight}

\subsubsection{Space Docking}

There are a number of mature applications of contact tasks from free-flight outside of decommissioning that could shed light on underwater applications, such as autonomous docking in space flight [39]. These studies recognize that although the two fields draw many parallels, there are a number of complications unique to subsea applications, such as the limited effectiveness of cameras or laser range finders. Additionally, the presence of unpredictable currents rival the control strategies used in space docking applications, which assume a passive and/or fixed docking station. While useful approaches to close-range navigation and safe design are proposed, many of the solutions assume that the point of docking can be designed beforehand to accommodate the vehicle, which is by no means assured when setting lifting points into scrap pieces of an offshore structure.

\subsubsection{Unmanned Aerial Vehicles (UAVs)}

Given the advancements in size reduction of UAV payload and manipulator weight, the field of autonomous aerial manipulation has begun to emerge under the impetus of enhancing mobility of operations for which ground robots do not suffice [36]. UAVs provide new analogs for free-flight manipulation relevant to AUV bracing. Particular studies of interest include the demonstration of a UAV capable of stably maintaining a constant force on a vertical wall while in free flight [1], and an autonomous helicopter equipped with a robotic hand for more generalized manipulation tasks [35]. UAVs such as these have taken significant steps forward in free-flight contact tasks by tackling generalizable problems such as dynamic coupling, computer vision techniques, and force feedback. However, the classic peg-in-hole problem has yet to be investigated from a free-flight UAV standpoint. Studies in fixed-base robotics have pointed towards programmable compliance to conquer the peg-in-hole problem [44], which, in conjunction with current UAV manipulation progress, may provide a controller-based 
solution to the free-flight peg-in-hole problem. Yet another viable solution is to avoid

the peg-in-hole problem all together. By taking a mechanical design approach, a free-flight bracing technique is proposed that consolidates the drilling, tapping, and affixing steps into one single penetration of the workpiece.

\subsection{Virdrill: A Two-Stage, Reversible and Remov- able, Drilling and Tapping Mechanism}

The peg-in-hole difficulty present in the application of decommissioning served as the motivation for designing an anchoring solution. Virdrill, a two-stage, reversible and removable, drilling and tapping mechanism, takes all necessary mechanical steps in attaching an anchoring point, and consolidates them into one penetration, shown in Figure 3-2. Virdrill enables a simple solution to attaching a lifting point, and opens the door to a new bracing method for underwater vehicles in situations where marine growth or complex geometries disallow the engagement of traditional grippers.

\subsection{Prototyping for Component Functionality}

In order to verify the functionality of Virdrill, a plastic prototype was fabricated solely from $3 \mathrm{D}$ printed parts. Although this prototype was not fabricated for its designed strength, the prototype established the geometrical feasibility of performing all mechanical steps within one single penetration. CAD renderings of a modified version of the AUV-ready Virdrill feature an enlarged key bit and tap sleeve, which enhance visibility and handling during in-hand demonstrations of the transition of torque between the drill bit and tap sleeve, shown in Figure 3-3. 


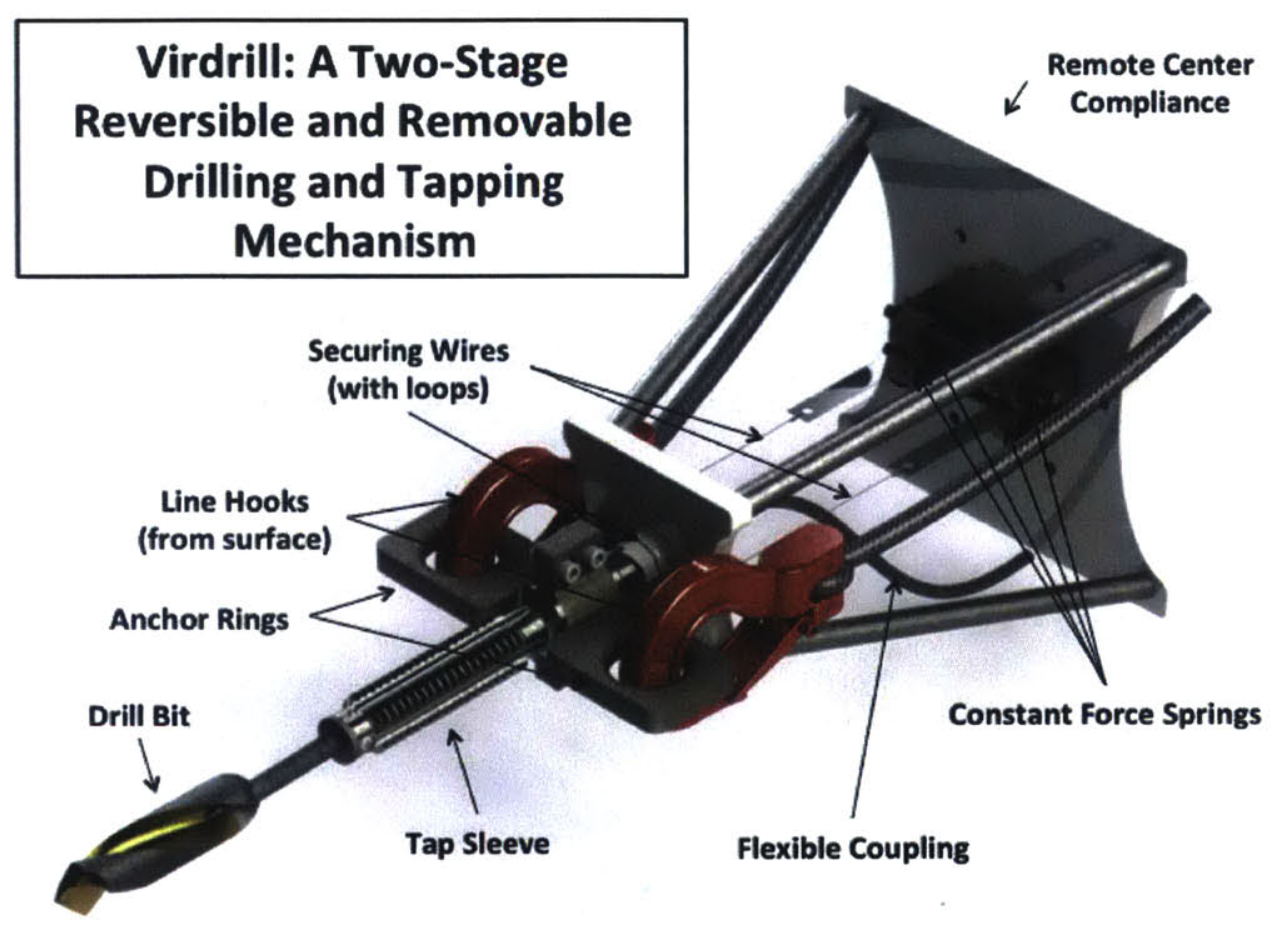

(a) Virdrill Isometric Cutout

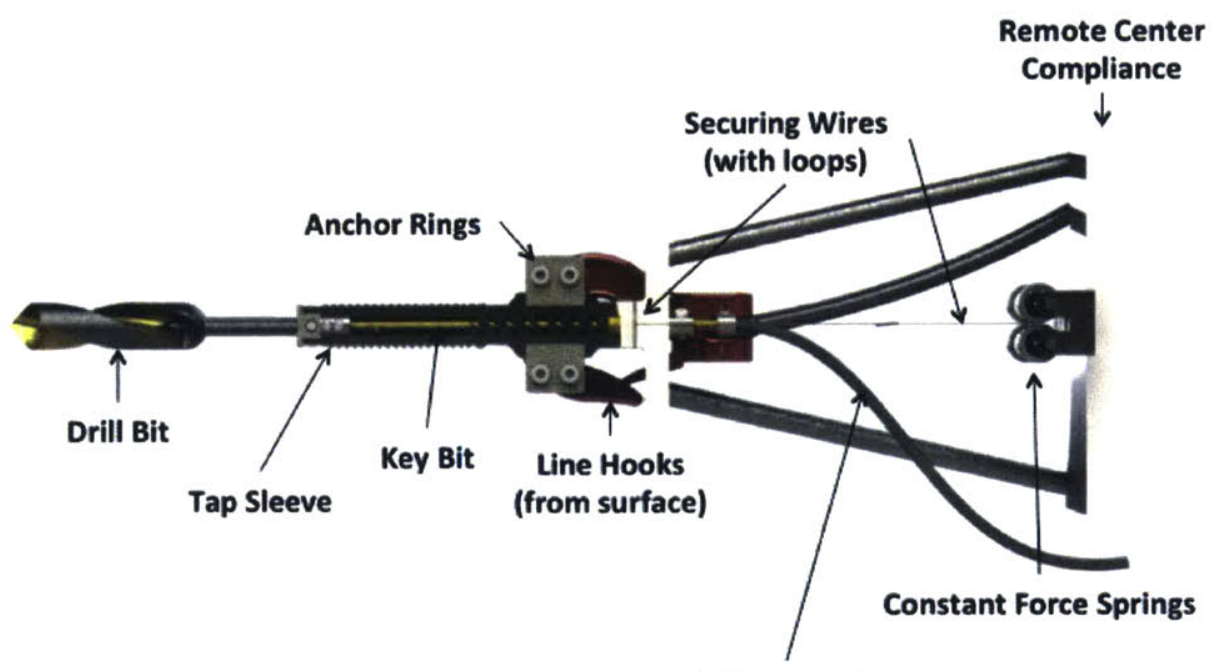

Flexible Coupling

(b) Virdrill Profile Cutout

Figure 3-2: An isometric (3-2a) and profile cutout (3-2b) CAD rendering of Virdrill integrates a Remote Compliant Center (RCC) with lifting lines attached. Virdrill also features spring-cocked securing wires, and a flexible coupling that transmits torque to the removable key bit. 


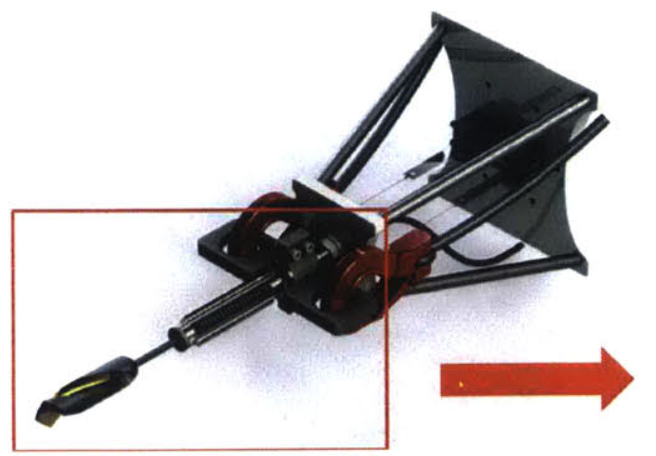

(a)

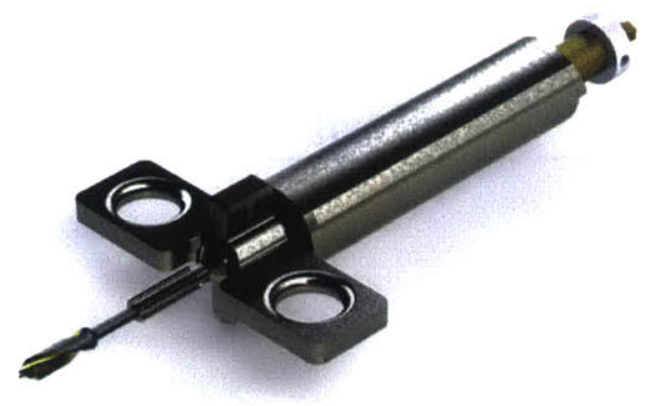

(b)

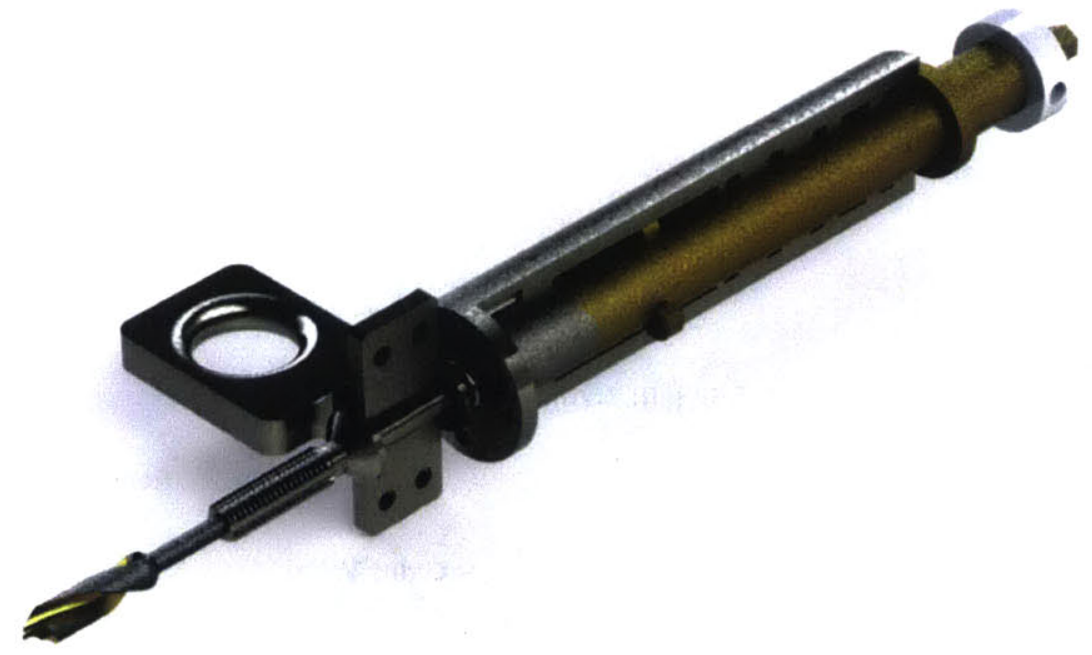

(c)

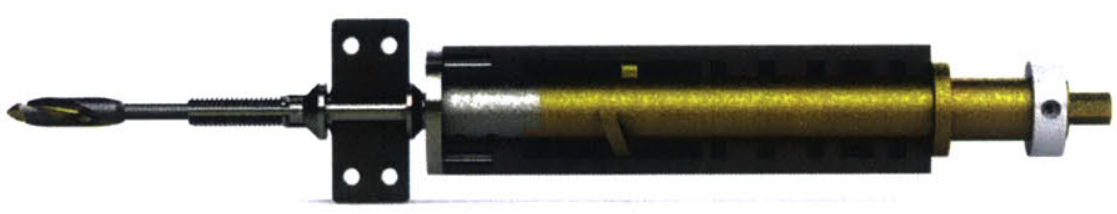

(d)

Figure 3-3: An isometric rendering of the AUV-ready Virdrill (3-3a) features a red box around the components isolated and modified for the demonstration-ready version (3-3b). An isometric cutout rendering of the demonstration-ready version shows an enlarged key bit and tap sleeve, and no longer features the line hooks, flexible coupling, and RCC (3-3c). A profile cutout rendering (3-3d) provides another view of the enlarged tap sleeve that helps to clearly demonstrate the transition of torque transition between the drill bit and tap sleeve. 


\subsubsection{Component Functionality}

\section{Key Bit}

Virdrill has four different rotating components; the first of which is the key bit, show in Figure 3-4. This key bit is the only portion of Virdrill that remains directly attached to the autonomous decommissioning vehicle throughout the entire anchoring process, and transmits both thrust and torque from the vehicle to the rest of the mechanism.

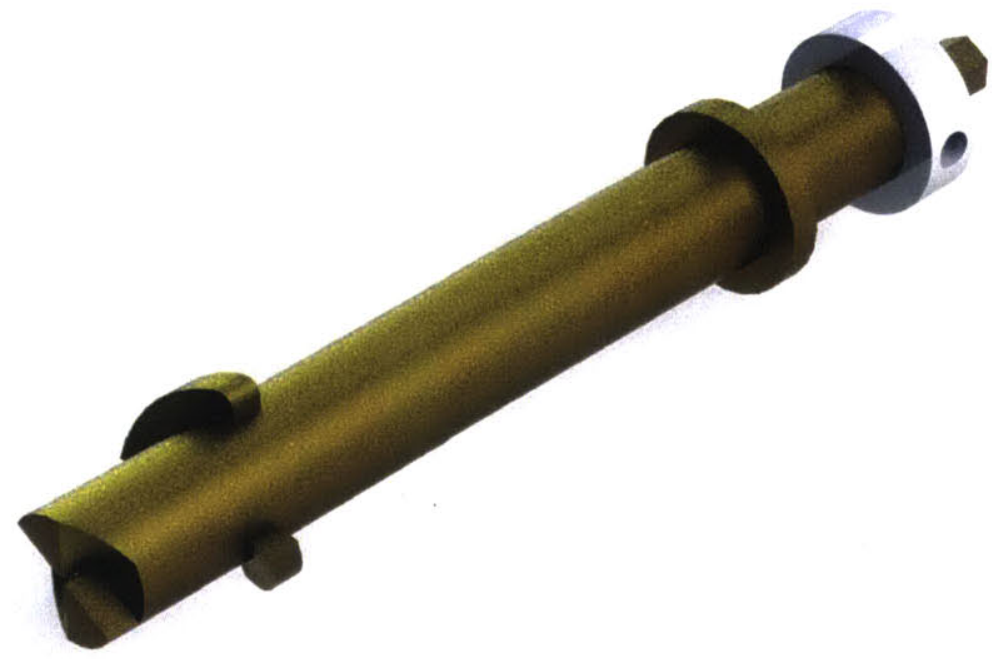

Figure 3-4: The right end of the key bit features a hexagonal shaft that functions as a generic point to which a torque-transmitting end effector may attach, and the gold and white rings are lock rings keep the key bit attached to the vehicle. Finally, both geometric features protruding from either the side or the end of the key bit shaft at the left end of the key bit transmit torque and thrust to either the drilling piece or the tapping piece.

\section{Drill Bit}

The second component is the drill bit, displayed in Figure 3-5. This drill bit has a complementary mating bit, which serves not only to interface with the key bit from which it is imparted torque, but also to restrain the drill bit from unintentionally being removed from the rest of the mechanism. The long shaft associated with the 
drill allows for it to create a pilot hole for the tap without having to be removed for the insertion of another tap, and therefore completely avoids the peg-in-hole problem.

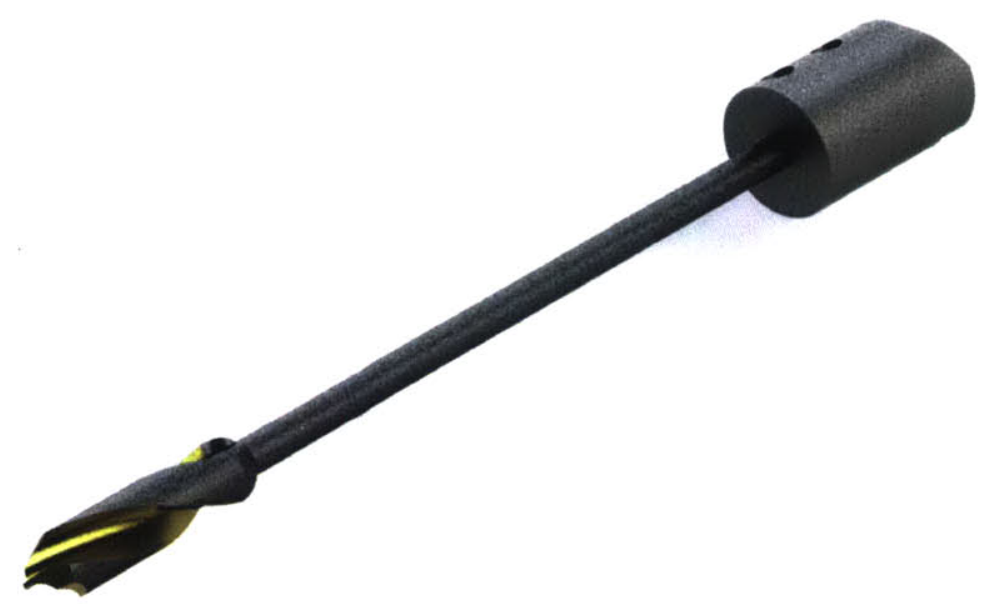

Figure 3-5: The right end of the drill bit piece features a detachable mating bit. While it is used to transmit thrust and torque from the key bit, the mating bit is also detachable to allow for its assembly into the entire mechanism. The length of the shaft allows the drill bit to telescope into the workpiece, while clearing the length of the tap sleeve. The left end of the drill bit piece features a half-inch drill that creates the pilot hole for the tap sleeve.

\section{Tap Sleeve}

The third component is the tap sleeve, shown in Figure 3-6, which is used to support the load of the cut piece, and to facilitate the mating between the key bit and drill bit. Incorporated within the tap sleeve are guiding grooves for the key bit, which axially offset midway down the length of the tap sleeve in order to create a surface through which the key bit can apply torque to solely the tap. This unique design allows for a decision as to where torque should be applied - either to drilling or to tapping. 


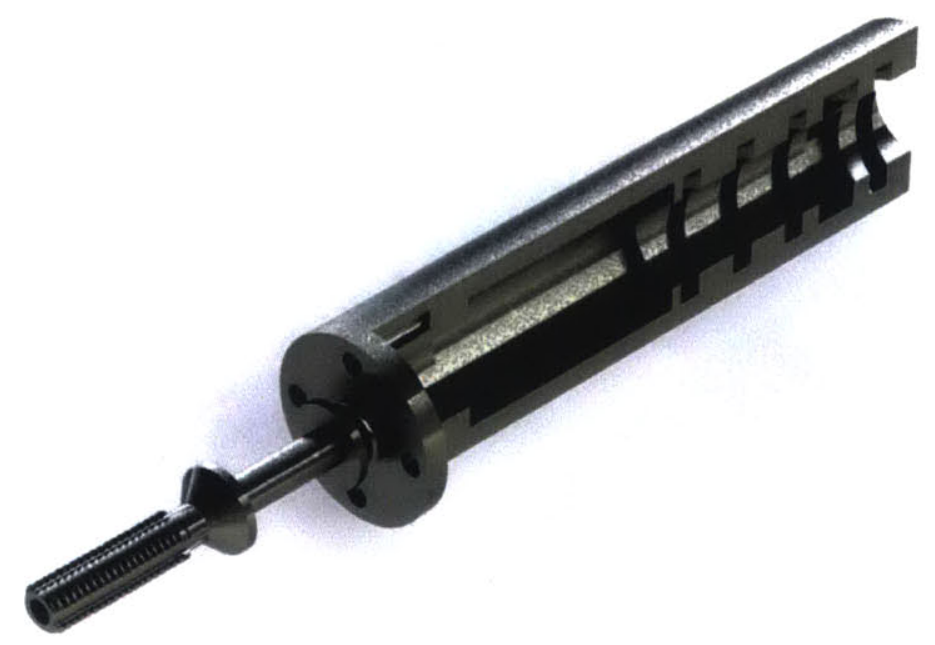

Figure 3-6: The tap sleeve serves as a transmission system for the key bit. The offset present in the internal spiraling groves at the right portion of the piece provide the key bit with a way of transmitting torque to either the drill bit or the tap, depending on the location of the key bit within the tap sleeve. The base of the cylindrical portion of the tap sleeve is where the actual tapping portion of the piece is attached. This feature is detachable to allow for the insertion of the drill bit and its fastening into the rest of the mechanism using the complementary mating bit.

\section{Anchor}

The fourth and final component is the anchor, illustrated in Figure 3-7, which attaches between the base of the cylindrical section of the tap sleeve, just above the tapping section. The anchor can rotate freely about this attachment point, independent of both the drill and the tap sleeve, thus allowing for mooring line hooks to remain attached to the anchoring mechanism during the entire drilling, tapping, and affixing process, without any issue of tangling.

It should be noted that there are other secondary parts in the current iteration of the prototype that facilitate the ease of attachment and assist in conveying a more complete story of a coupling between. A flexible coupling is attached to the base of key bit in order for allow for off-axis torque transmission to the mechanism, thus opening versatility in the viable arrangements of a potential torque source on the AUV. Additionally, spring-tensioned wires are attached to the anchor rings, which help keep the tap sleeve stationary while the key bit traverses through its transition 


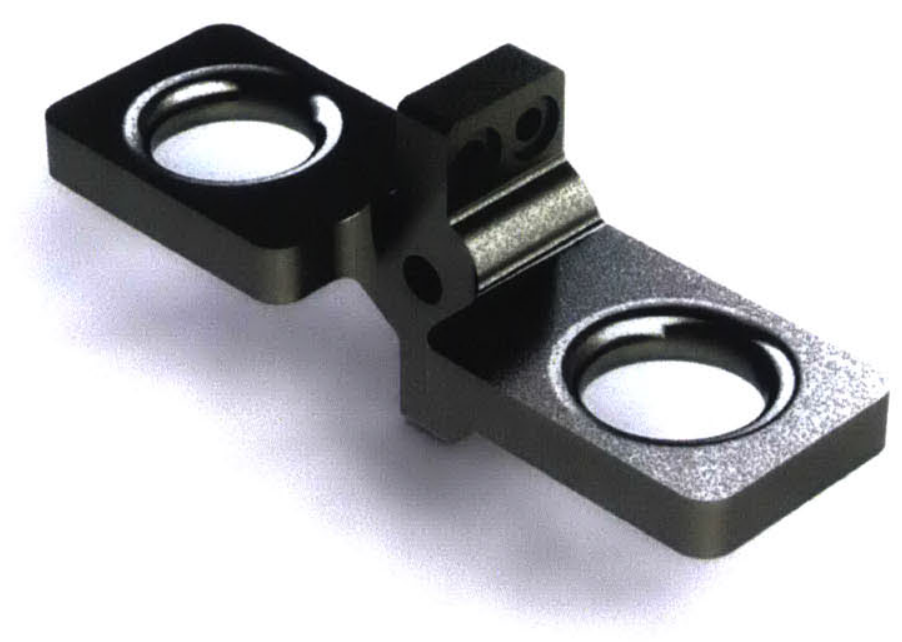

Figure 3-7: The anchor is comprised of two separate and symmetrical pieces that attach around the base of the tap portion of the piece. There is enough clearance between the anchor and the tap sleeve to allow for its independent rotation from the rest of the mechanism. In the situation where the lifting lines are attached to the mechanism equipped onto vehicle before deployment, the insertion of the tap sleeve without the anchor's free rotation would result in the entanglement of the lines.

grooves. When the anchoring processes are complete and the vehicle pulls away, the anchor rings slip the tensioned wires off of the rest of the assembly, thus freeing the vehicle from the anchor. Finally, incorporating remote center compliance helps to prevent undesirable torqueing or jamming from the motions of the AUV by allowing for small planar motions perpendicular to the axis of the assembly, while still allowing for the axial translation of thruster.

\subsubsection{Drilling}

When the cut piece is met, the key bit provides both thrust and torque solely to the drill bit, shown in Figure 3-8a. The drill bit penetrates into the piece until the tap sleeve reaches its surface, seen in $3-8 \mathrm{~b}$. At this point, the drill bit begins to telescope even further into piece, as in Figure 3-8c, thus allowing for penetrating room for the tap. 


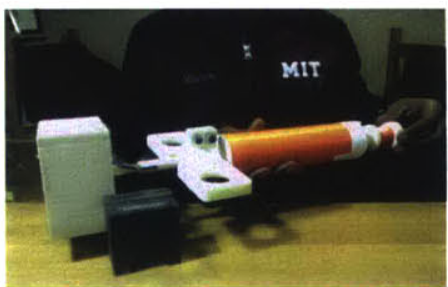

(a)

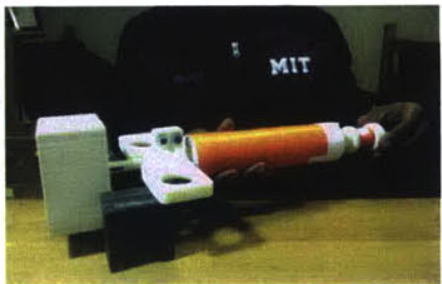

(b)

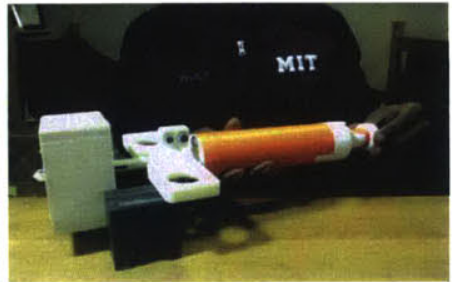

(c)

Figure 3-8: Key points in the drilling step include the initial penetration of the drill bit (3-8a), the full telescoping of the drill bit (3-8b, 3-8c).

\subsubsection{Tapping \& Anchoring}

Once the drill bit has been fully telescoped, the anchor must be tapped into the cut piece via the tap sleeve. In order to disengage the drill bit, the key bit must be simultaneously pulled and turned counter clockwise, depicted in Figure 3-9a. This allows for a separation from the drill bit, and an engagement of the guide grooves within the tap sleeve. As the key bit is pulled away and turned counterclockwise, it eventually reaches the axial offset of the guide grooves, shown in Figure 3-9b. At this point, the key bit must be pushed forward and then both turned clockwise and pushed forward in order to transmit torque to the tap sleeve through the surface created by the guide groove offset, as seen in Figure 3-9c.

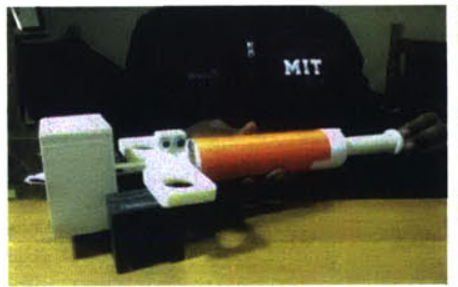

(a)

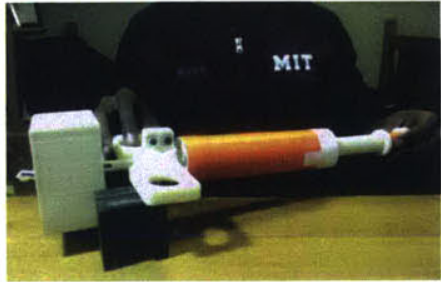

(b)

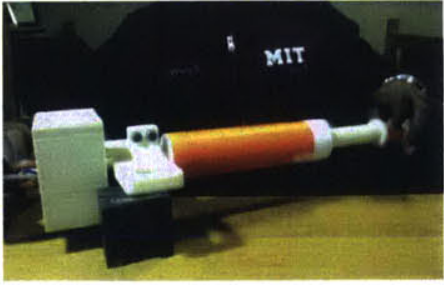

(c)

Figure 3-9: Key points in the tapping step involve the disengagement of the key bit by pulling onto the key bit and turning counterclockwise (3-9a), and, once the internal guide groove offset is met (3-9b), pushing into the key bit and turning clockwise (3-9c). 


\subsubsection{Removing}

Once the tap has been fully penetrated into the cut piece, and the anchors are flush with its surface, shown in Figure 3-10a, the key bit is ready to be removed. While still pushing forward but instead turning counterclockwise, the key bit continues on the axially offset guide groove until it is guided out of the tap sleeve, as in Figure $3-10 \mathrm{~b}$, thus decoupling the vehicle from the anchor and completing the anchoring task, shown in Figure 3-10c.

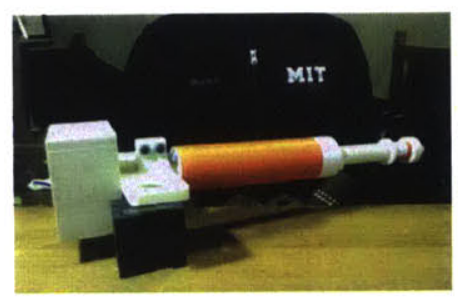

(a)

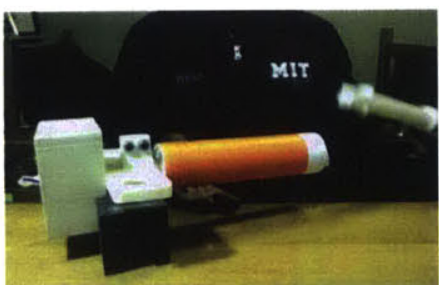

(b)

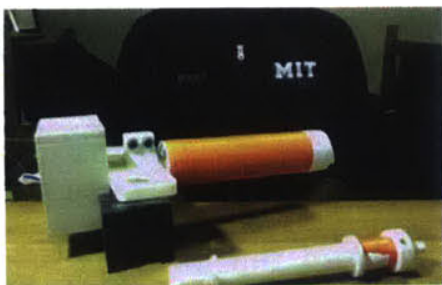

(c)

Figure 3-10: Key points in the removing process start with the anchor completely flush with the workpiece surface (3-10a), after which the key bit is pushed into the mechanism and turned counter clockwise to guide the key bit out (3-10b), and thus, the key bit, which is still attached to the vehicle, has been completely separated from the anchor (3-10c).

\subsection{Conclusions and Future Work}

Virdrill provides a novel and simple method for creating an anchoring point from an AUV in free-flight. The ability to fabricate such a device has been made possible from advances in 3D printing, which has allowed for complex geometries to be generated on small scales by using additive methods, as opposed to traditional subtractive methods such as using mills, lathes, and cutters. Now that geometrical feasibility has been validated, the next step would be to fabricate a metal version of Virdrill, so that strength requirements can also be tested for validation. This future work would involve even more advanced methods of 3D printing, specifically by implementing direct metal laser sintering (DMLS), which is an additive technique in manufacturing that utilizes a high powered laser to sinter powdered metal. With the help of DMLS 
technology, a full-functioning final prototype of Virdrill could be fabricated, outfitted onto a vehicle, and tested for a comprehensive proof of concept. 


\section{Chapter 4}

\section{Autonomous Manipulation of Subsea Structures from a Free-Floating}

\section{Vehicle}

\subsection{Key Manipulation Tasks for Preliminary Phases of Decommissioning}

The previous chapter discussed a novel mechanical solution to installing anchoring points from free-flight, which sheds light on the possibility to provide bracing while the AUV performs other contact tasks. However, several crucial tasks in decommissioning exist where penetrating the structure may not be desirable, such as removing biofouling from complex geometries to perform non-destructive testing (NDT), or evaluating the functionality of valves and levers [25]. Even in cases where it is possible to brace onto the structure, the ability to interact without bracing allows for the use of cheaper and simpler vehicles that are equipped with only one manipulator.

In situations where a bracing point may not be available and penetrating the structure to create a bracing point is undesirable, the interactions between the vehicle and the environment must be accomplished from free flight, which suggests the use of impedance control to better mimic the desirable dexterity of humans. Al- 


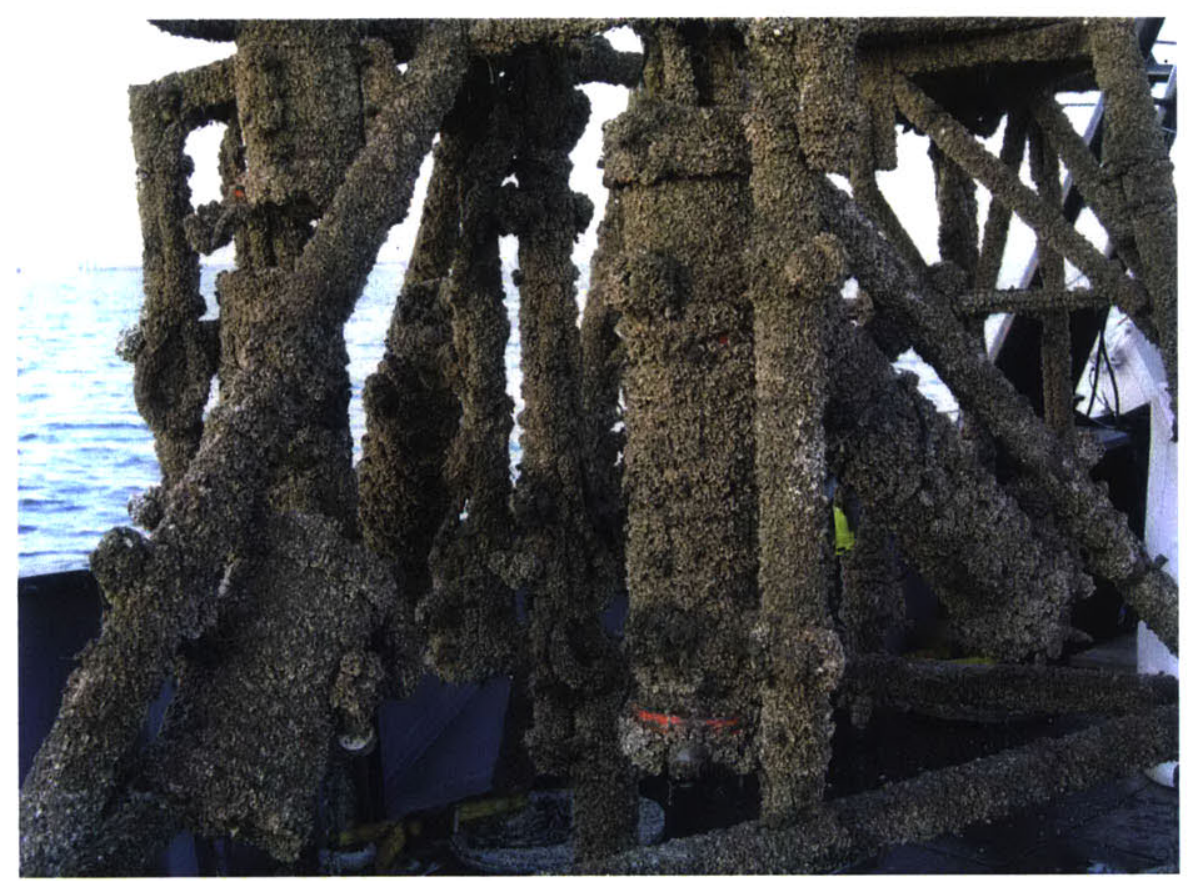

Figure 4-1: After several months of exposure to marine life, heavy biofouling develops on offshore structures. Such biofouling is an obstacle to thorough inspections and non-destructive testing [54].

though saturation dives can achieve depths at 1,000 feet for 30 days at a time, these dives push against the physical limitations of the human body, forcing work at any deeper depths to be done by ROVs [3]. At these depths, position-feedback has traditionally dominated, the most obvious example of which being an ROV operator performing contact tasks solely by sight of the end effector via a live video feed. Unfortunately, this method does not capture the relationship between position and force, i.e., impedance control, which is naturally exhibited in human diver manipulation, and allows for contact tasks to be performed in a safer and more efficient manner [30]. In an attempt to retain the same dexterous capabilities afforded by commercial divers in shallower water for unmanned vehicles used at deeper depths, this chapter highlights steps taken to augment position-controlled robotic manipulators that are typically used in industry with impedance control capabilities. 


\subsection{Using Passivity to Promote Robustness in Envi- ronment Interactions}

The appeal for a passive system is that it may consume energy, but does not produce energy, so if two passive systems interact, their interactions are guaranteed to be stable [18]. For this reason, passivity presents itself as an attractive candidate around which to design a control law for a robotic manipulator; ensuring passivity in a robotic system is a strong path towards robustness against the destabilizing effects of contact.

Dynamics of physical systems satisfy the following energy conservation equation:

$$
\frac{d}{d t}[\text { Stored Energy }]=[\text { External Power Input }]+[\text { Internal Power Generated }]
$$

More generally:

$$
\dot{V}(t)=y(t)^{T} u(t)-g(t)
$$

where $u$ is input "effort" (e.g. force), and $y$ is output "flow" (e.g. velocity), and $\dot{V}(t)$ and $g(t)$ are scalar functions of time.. A system can be defined as passive if there exists a lower bounded $V$, where $g=0$ [50]. Conceptually, this means that a finite amount of energy can be dissipated, but no energy can be created.

\subsection{Experimental Setup}

\subsubsection{Overall System Layout}

In order to investigate the possibilities of having an autonomous underwater vehicle equipped with a non-backdrivable end effector interact passively with its environment, Dexter, an autonomous surface vehicle (ASV), was fabricated and outfitted with two ROV thrusters, and a 5-DOF robotic arm attached to the vehicle via a force/torque sensor, shown in 4-2a. A simplified version of this setup is modeled with a mass, $m$, and its associated damping, $b$, representing the mass and damping of the vehicle submerged in water with the thruster force, $f_{\text {thruster }}$, acting upon it, in Figure 4- 
2a. The robotic arm is treated as an ideal velocity source, $v_{a r m}$, due to its nonbackdrivability from a high gear reduction. The mass, $m_{2}$, and its associated damping, $b_{2}$, represent the mass and damping of the end effector - whatever tool it may be submerged in the water with external force, $f_{\text {ext }}$, acting upon it.

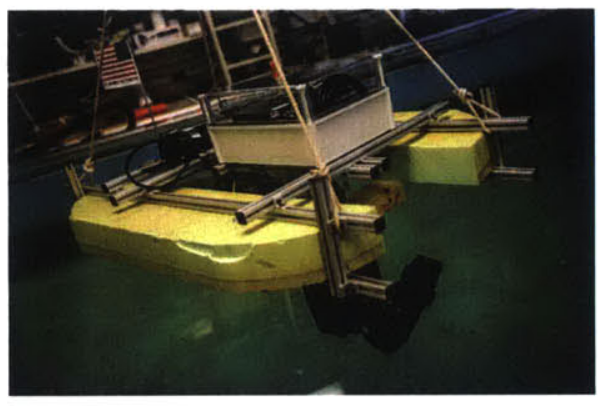

(a)

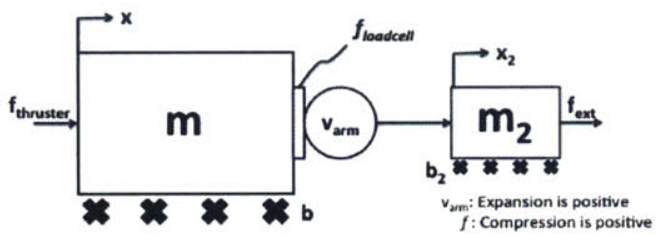

(b)

Figure 4-2: A picture (4-2a) and model (4-2b) of Dexter, the Autonomous Surface Vehicle. Its pontoon-like structure is comprised of two symmetric sections of highdensity foam for buoyancy, supported by an 80/20 aluminum frame that serves as a modular system for mounting hardware.

Several major problems were encountered while using the manipulators, which led to three unique iterations of the vehicle with a different manipulator for each. The first iteration, shown in Figure 4-2a, incorporated the CISP ECA 5E Micro Arm, which ultimately suffered from permanent motor failure in two crucial joint actuators. Given that initial experimentation was restricted to one dimension, the second and third iterations of Dexter ASV used Firgelli Automations linear actuators. Using Firgelli Automations' High Speed Linear Actuators (Figure 4-3) allowed for a larger range of possible achievable impedances. Unfortunately, not enough force could be generated from the system in order to mitigate friction forces or the external loads placed on the actuators, and retain the ideal velocity source assumption. The third and current iteration of the vehicle uses Firgelli Automations' Light Duty Rod Actuator (Figure 4-4), which exhibited an acceptable showing of both actuator speed and non-backdrivability. 


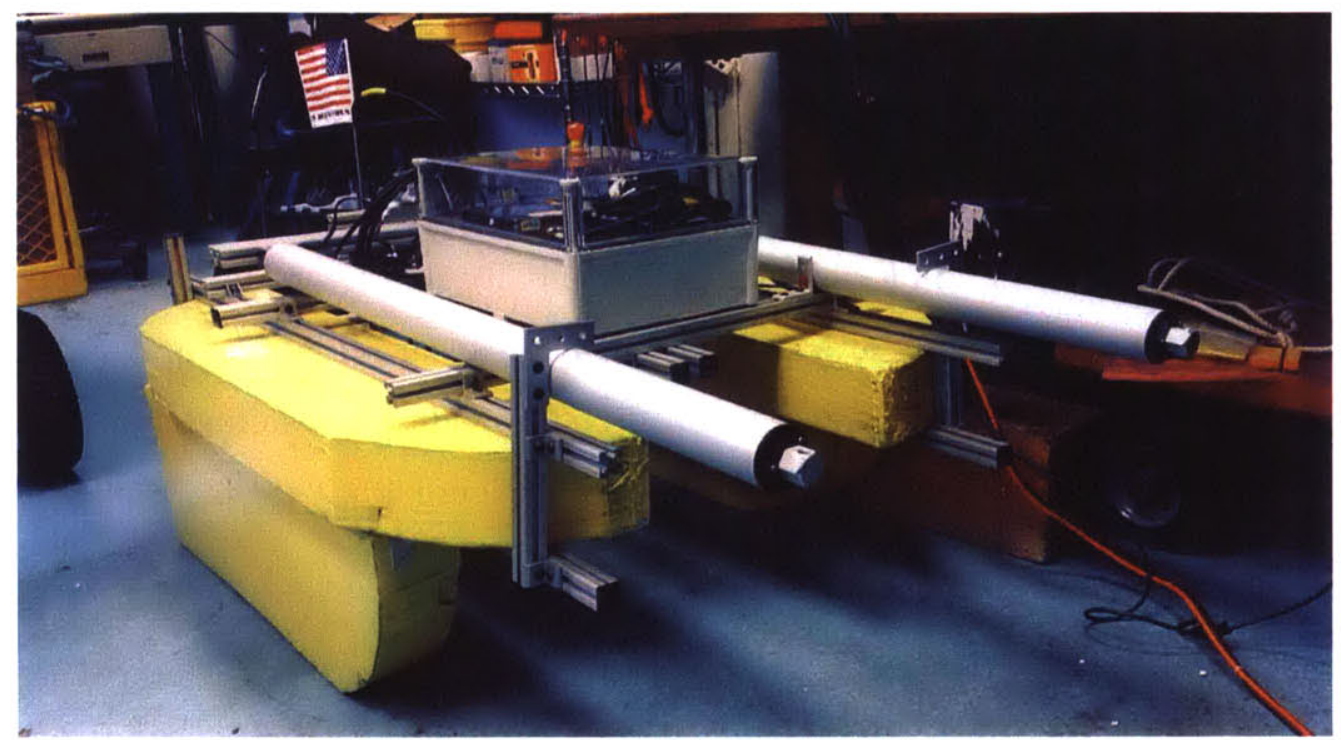

Figure 4-3: The second iteration of Dexter ASV is equipped with two Firgelli Automations High Speed Linear Actuators, and shows the addition of flotation to create more freeboard.

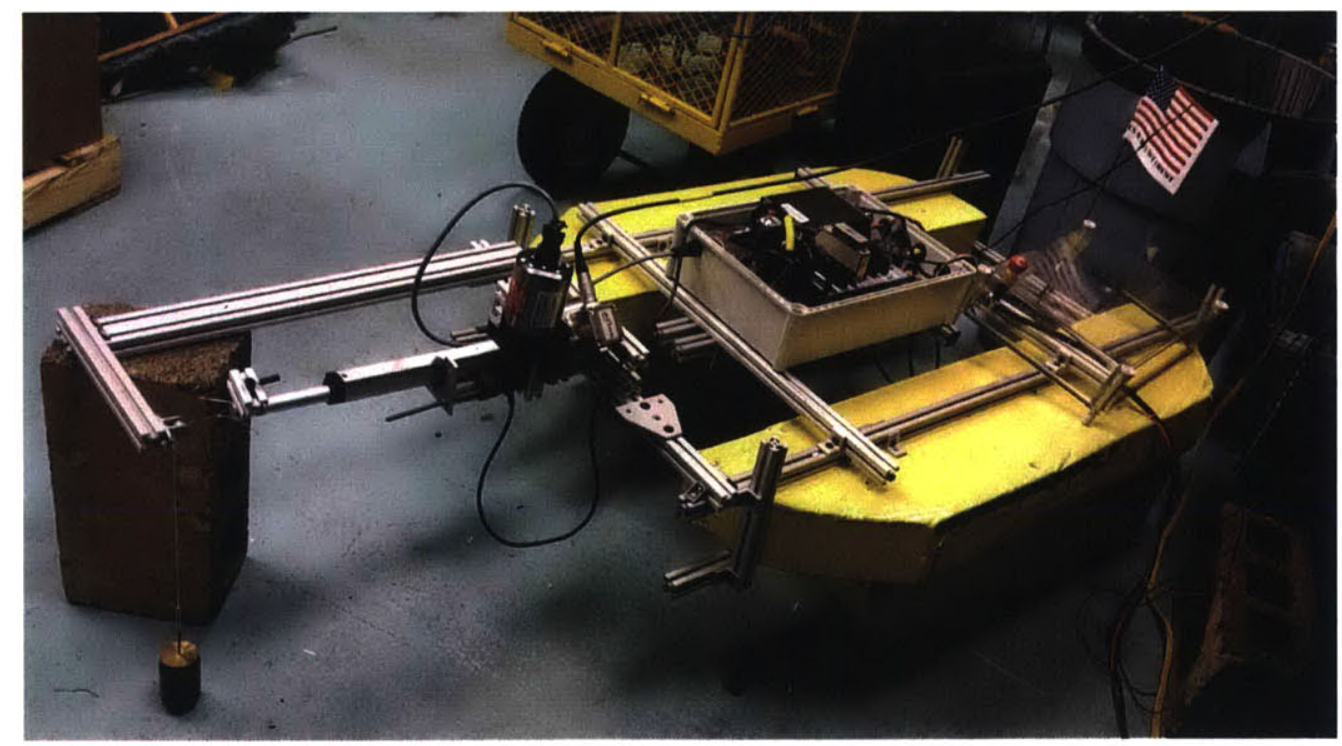

Figure 4-4: The third iteration of Dexter ASV features a single linear actuator with acceptable stroke, speed, and non-backdrivability. 


\subsubsection{Hardware}

The linear actuator on Dexter ASV is the Firgelli Automations Light Duty Rod Actuator with a $6 "$ stroke length, stroke speed of $0.5 \% / \mathrm{s}$, and a $667 \mathrm{~N}$ maximum dynamic force (Figure 4-6a). It was chosen for its ability to simplify the analysis to solely one dimension (axially), for its non-backdrivability, and for its actuation speeds comparable to those of the ECA arm. The design of the linear actuator simply features a DC motor, a gear reduction, and a worm drive, providing few failure modes and ease of deconstruction/reconstruction, illustrated in Figure 4-5.

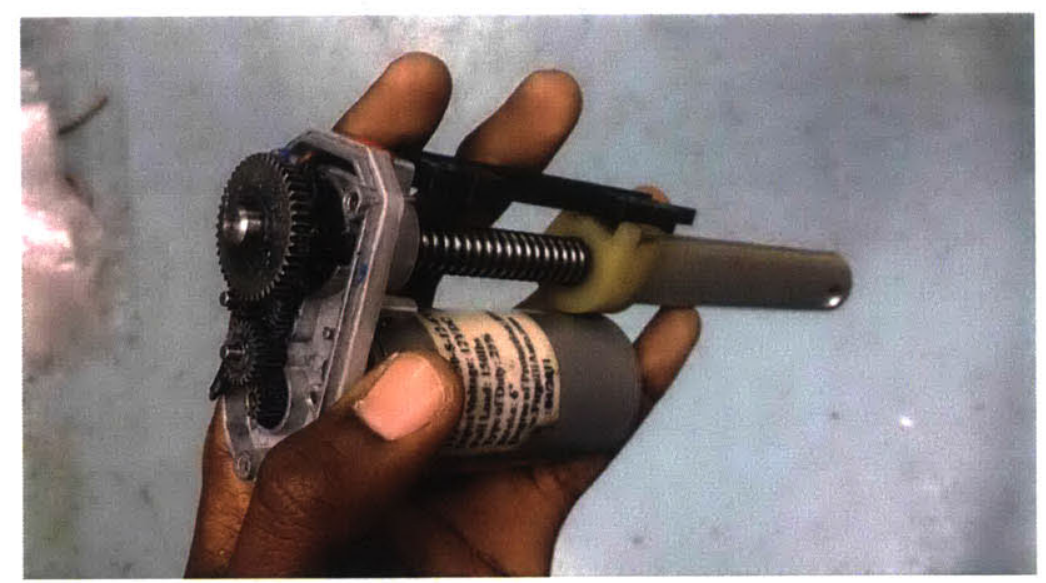

Figure 4-5: Dissection of the linear actuator shows the simple components that comprise the mechanism.

Force sensing was made possible using the ATI SI-660-60 Force/Torque Sensor, shown in Figure 4-6b, with the front end attached to the base of the linear actuator, and the back end fixed to the stationary raft. This load cell is capable of measuring forces/torques in six degrees of freedom, but only forces in only one dimension (axially) were taken into account. The ATI SI-660-60 Force/Torque Sensor features a maximum axial load of $1980 \mathrm{~N}$, resolution of $0.25 \mathrm{~N}$, and a bandwidth of at least 200 $\mathrm{Hz}$.

Unimeasure's HX-P510 String Potentiometer was incorporated into the experimental setup in order to compare the end effectors actual position and velocity against the model's predictions (Figure 4-7a). It features a $200 \mathrm{~cm}$ stroke length, 5-10 VDC output range, and a wire tension of 5.8 N. Additionally, the incorporation 


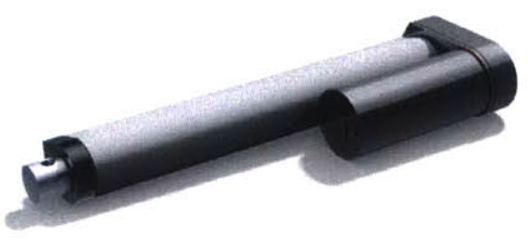

(a)

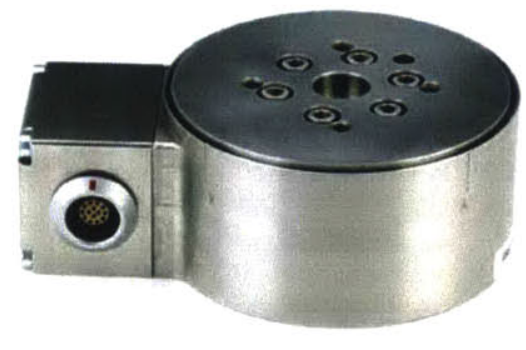

(b)

Figure 4-6: Firgelli Automations Light Duty Rod Actuator (4-6a) [37] and ATI SI660-60 Force/Torque Sensor (4-6b) [24].

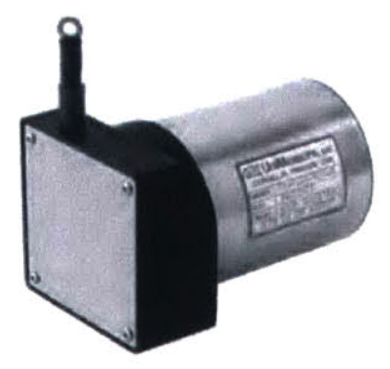

(a)

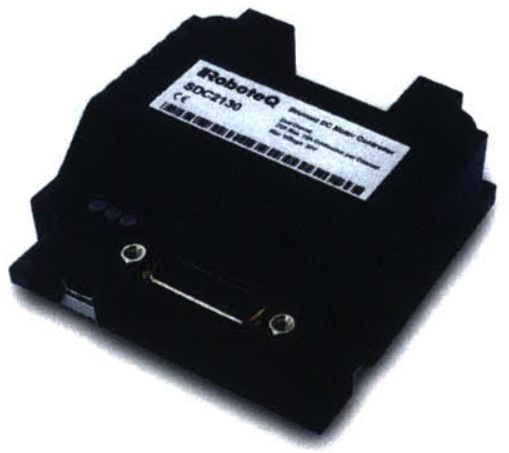

(b)

Figure 4-7: Unimeasure HX-P510 String Potentiometer (4-7a) [32] and RoboteQ SDC2130 Motor Controller (4-7b) [48]. 
of this string potentiometer provides the opportunity to provide state feedback to the system model in the future.

The linear actuator is controlled by the RoboteQ SDC2130 motor controller, shown in Figure 4-7b, a brushed DC motor controller that also comes equipped with $0-5 \mathrm{~V}$ analog inputs, one of which is used to transmit the analog position data coming from the string potentiometer to MOOS-IvP.

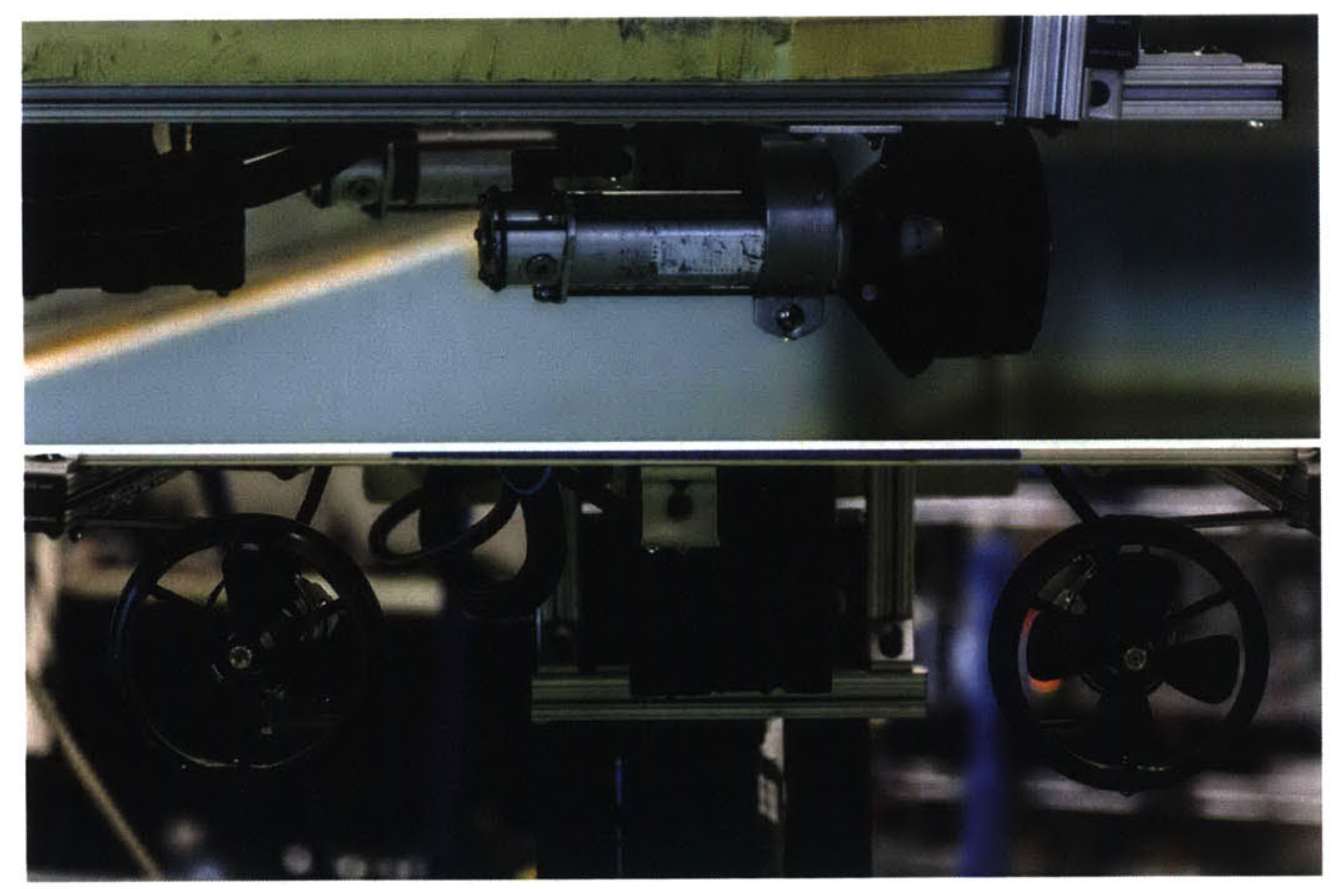

Figure 4-8: A profile view (top) and rear view (bottom) of the thrusters show two Inuktun SM3300-4 ROV Thrusters mounted to the aluminum frame on the bottom of the raft.

The RoboteQ motor controller also commands the two Inuktun SM3300-4 ROV Thrusters on the raft (Figure 4-8), which are rated for 150 watts at 24VDC. Since the configuration of these thrusters is asymmetric, a maximum of $59 \mathrm{~N}$ of force are provided in the forward direction, while a maximum of $11 \mathrm{~N}$ of force is provided in the reverse direction. The design of the thruster is simply comprised of a ducted propeller attached to the shaft of a brushed DC motor in a waterproof housing. 


\subsubsection{Software and Communication}

Although data handling was conducted primarily through MATLAB, several different interfaces were used in order to integrate the data from the separate sensors; a diagram of the communication architecture is shown in Figure 4-9.

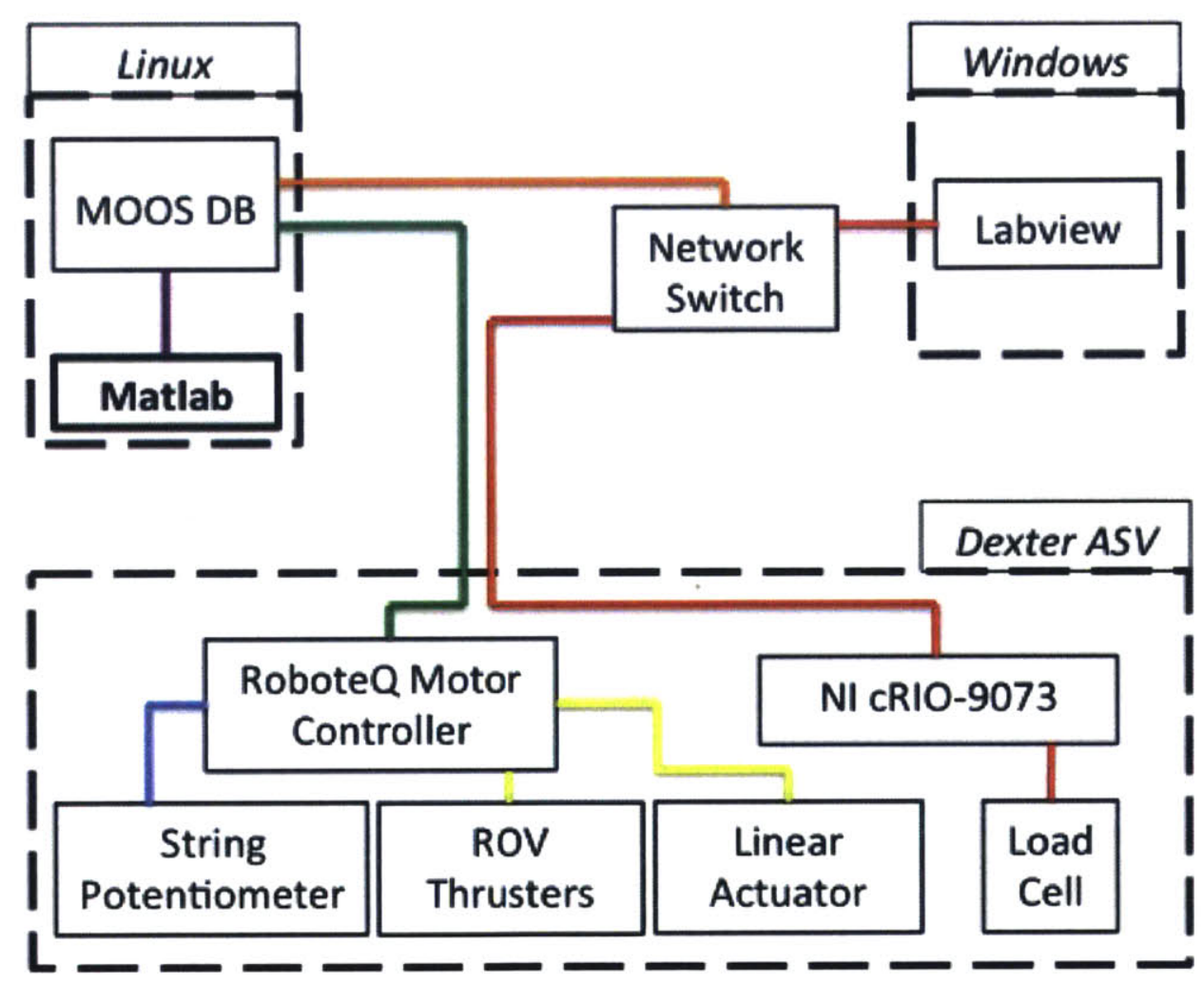

Figure 4-9: Visualization of the communication architecture of the Dexter ASV System, which is divided into three main components: The Linux machine, the Windows machine, and the raft.

There are three distinct components in the communication architecture of the Dexter ASV system: The Linux machine, the Windows machine, and the raft. All sensor inputs and controller commands are shuttled through MOOS-IvP on the Linux machine. MOOS-IvP acts as a centralize database, through which variables can be fetched (i.e. read) and notified (i.e. written) in coordination between multiple platforms in real-time [4]. MOOS-IvP also handles communication with the RoboteQ motor controller (Figure 4-10), which connects to the Linux machine via USB connec- 
tion. However, configuring the motor control must be done on Windows, as illustrated in Figure 4-11.

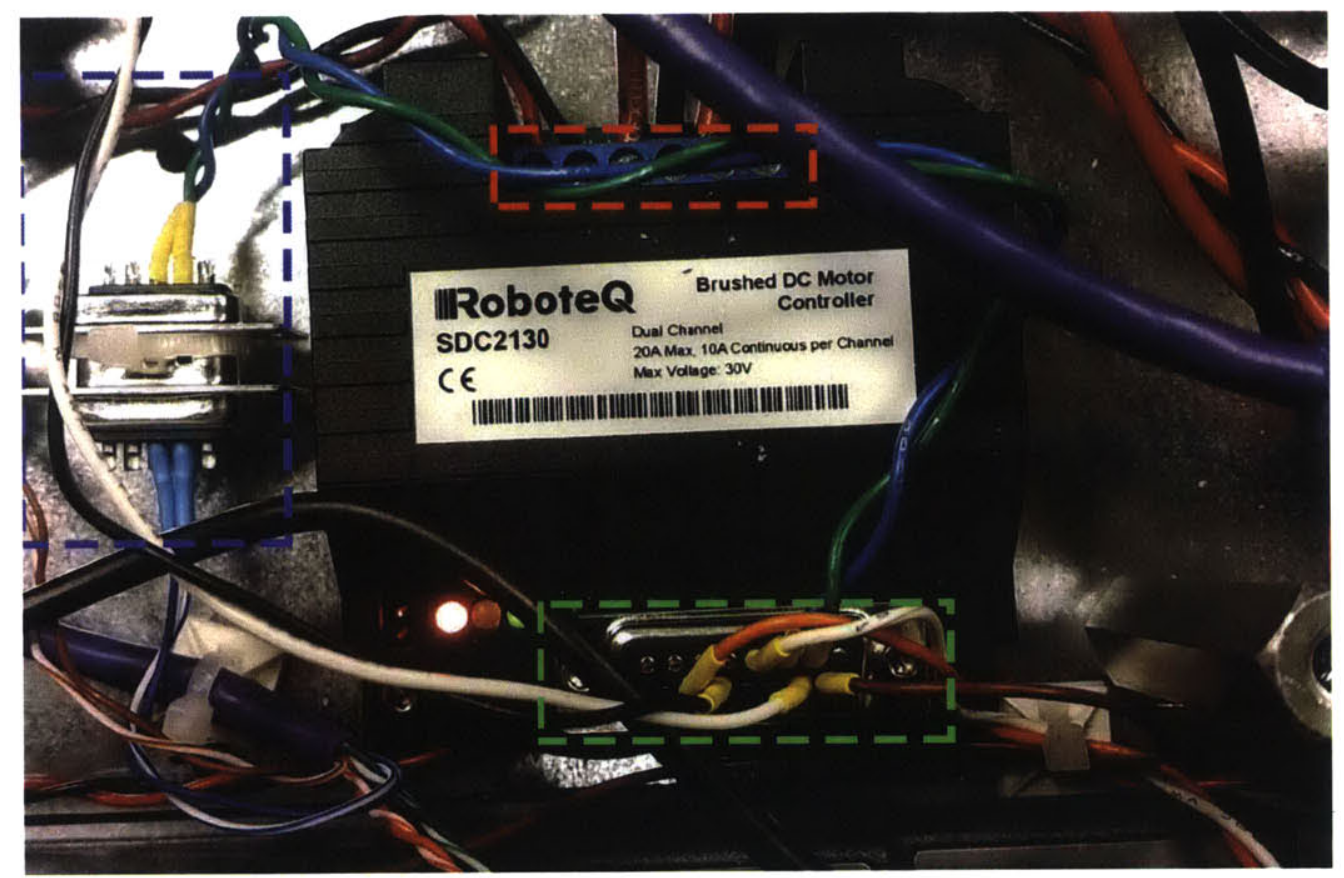

Figure 4-10: The RoboteQ SDC2130 Motor Controller acts as a hub through which motor commands are sent to either the linear actuator or the thrusters (red box), or analog string potentiometer data is received (green box). Communication is made with the Linux machine via USB connection (blue box).

The ATI load cell communicates directly to Labview on the Windows machine via the NI cRIO-9073 using the NI 9205 16-Bit Analog Input Module (Figure 4-12). Labview then sends the load cell readings to MOOS-IvP via a TCP/IP connection at a rate of $100 \mathrm{~Hz}$ (Figure 4-13). The RoboteQ motor controller sends position information from the string potentiometer and receives commands from MATLAB to send to the motors at a rate of $50 \mathrm{~Hz}$.

\subsection{Analysis of Manipulator on Fixed-Base in Air}

Although Dexter ASV in its entirety illustrates a bigger picture of the free-floating system designed to interact with its environment passively, initial interaction experiments were conducted on a fixed linear actuator. The fundamental concepts 


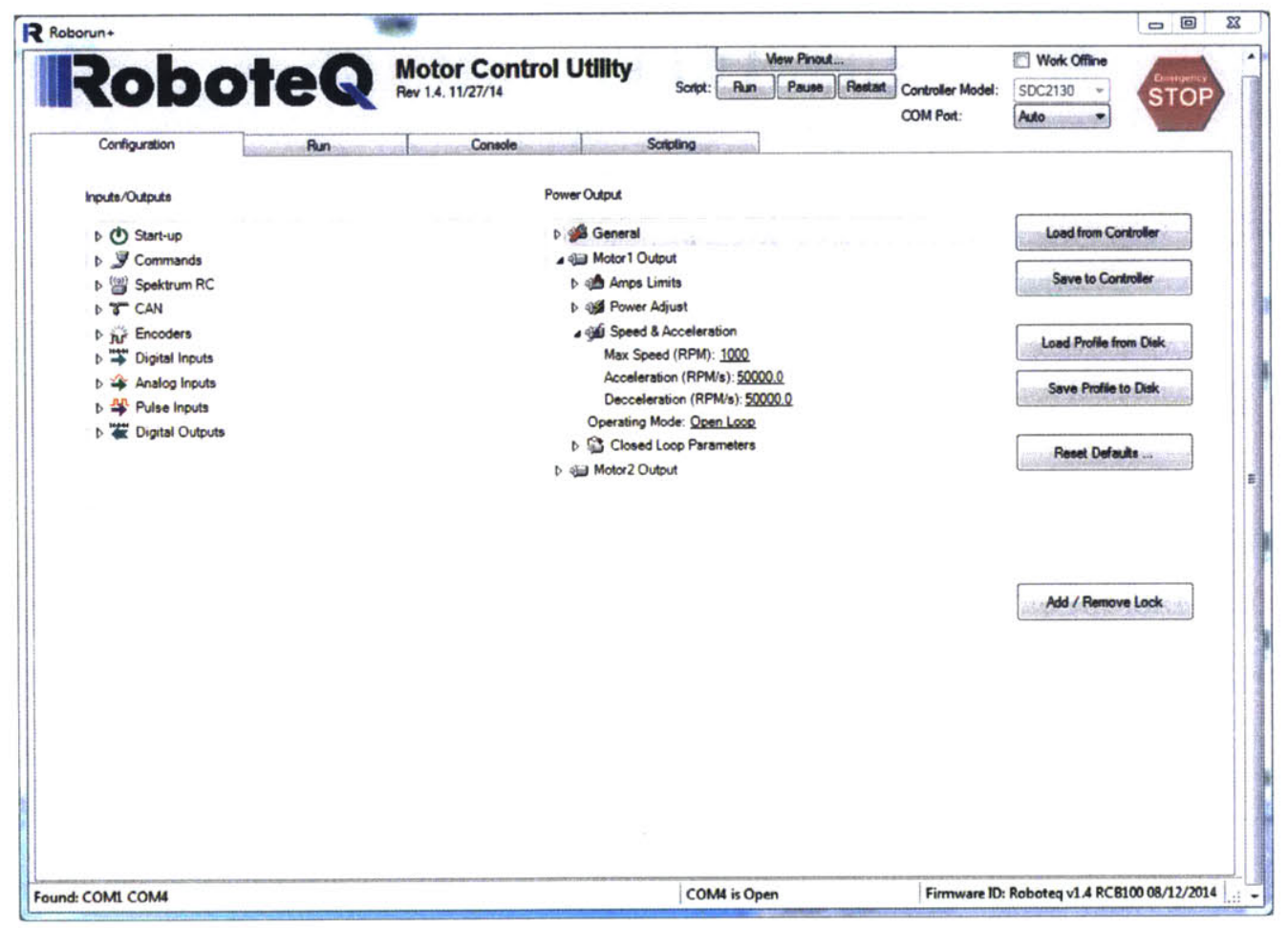

Figure 4-11: The RoboteQ Motor Control Utility provides options for closed loop control, but was set to "Open Loop" in order to give complete controller authority through MATLAB. Values for max speed, acceleration, and deceleration must be provided to the utility, but the effects of these values are negligible for the frequency ranges considered in experimentation. 


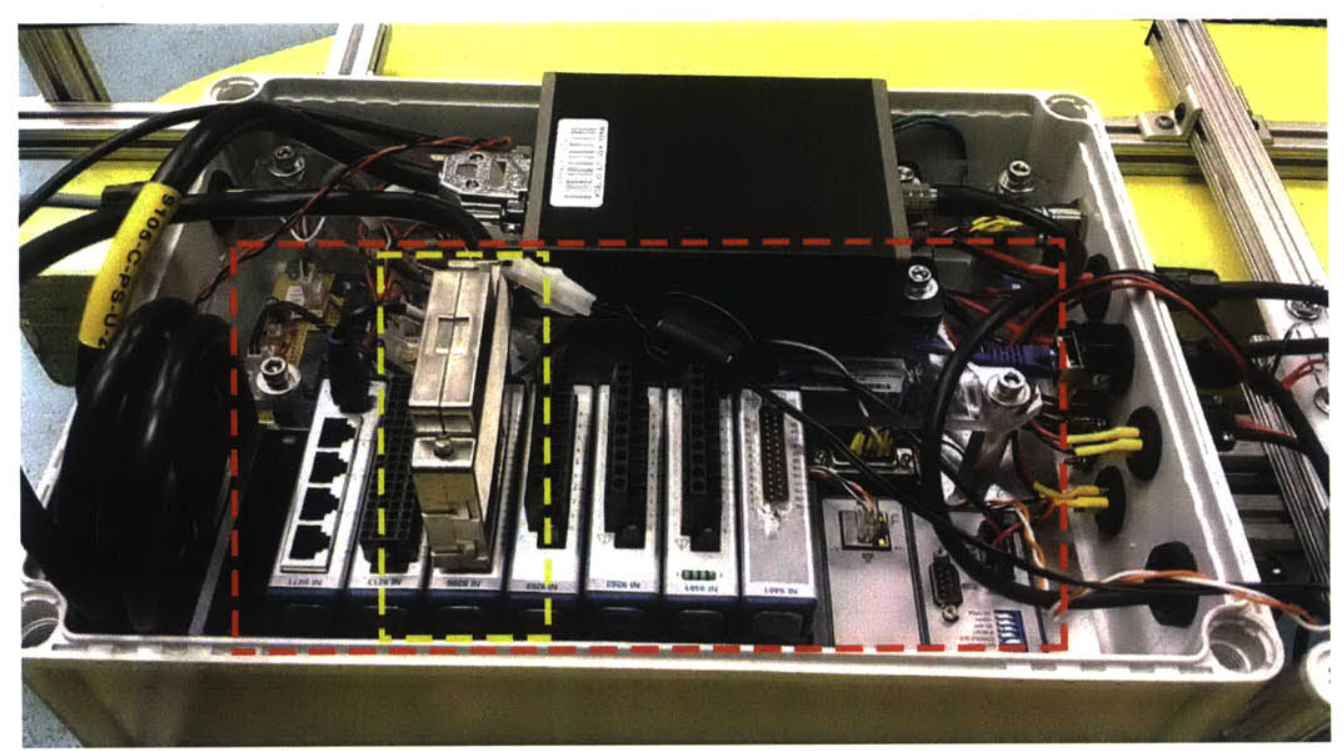

Figure 4-12: Within the raft's electronics box is the NI-cRio-9073 (red box) equipped with the NI 9025 16-bit Analog Input Module, which connects to the ATI SI-660-60 Force/Torque Sensor (yellow box).

researched by analyzing solely the linear actuator draw strong ties to the analysis of the overall raft-robot system. Developing a control law to have the manipulator interact passively sets the framework to create control laws to have a robot's interactions simulate human movements, e.g., the tasks that divers perform whom are hoped to be replaced by autonomy.

\subsubsection{Modeling the Linear Actuator}

A fairly simple model can be used to describe a non-backdrivable linear actuator. At first, a model-based approach was taken, treating the linear actuator as a free mass, $m$, attached to a fixed velocity source, $v_{\text {arm }}$, directly attached to load cell reading force, $f_{\text {loadcell }}$ or $f_{l}$ (Figure 4-14a). However, after several tests, it was demonstrated that the change in force due to the actuators acceleration was not discernible from noise, and therefore made the actuator's mass negligible. This further simplified the model by removing the free mass term and absorbing any mass associated with the base of the linear actuator into the mass of raft, or in this particular analysis, neglecting it altogether as part of the fixed base. 


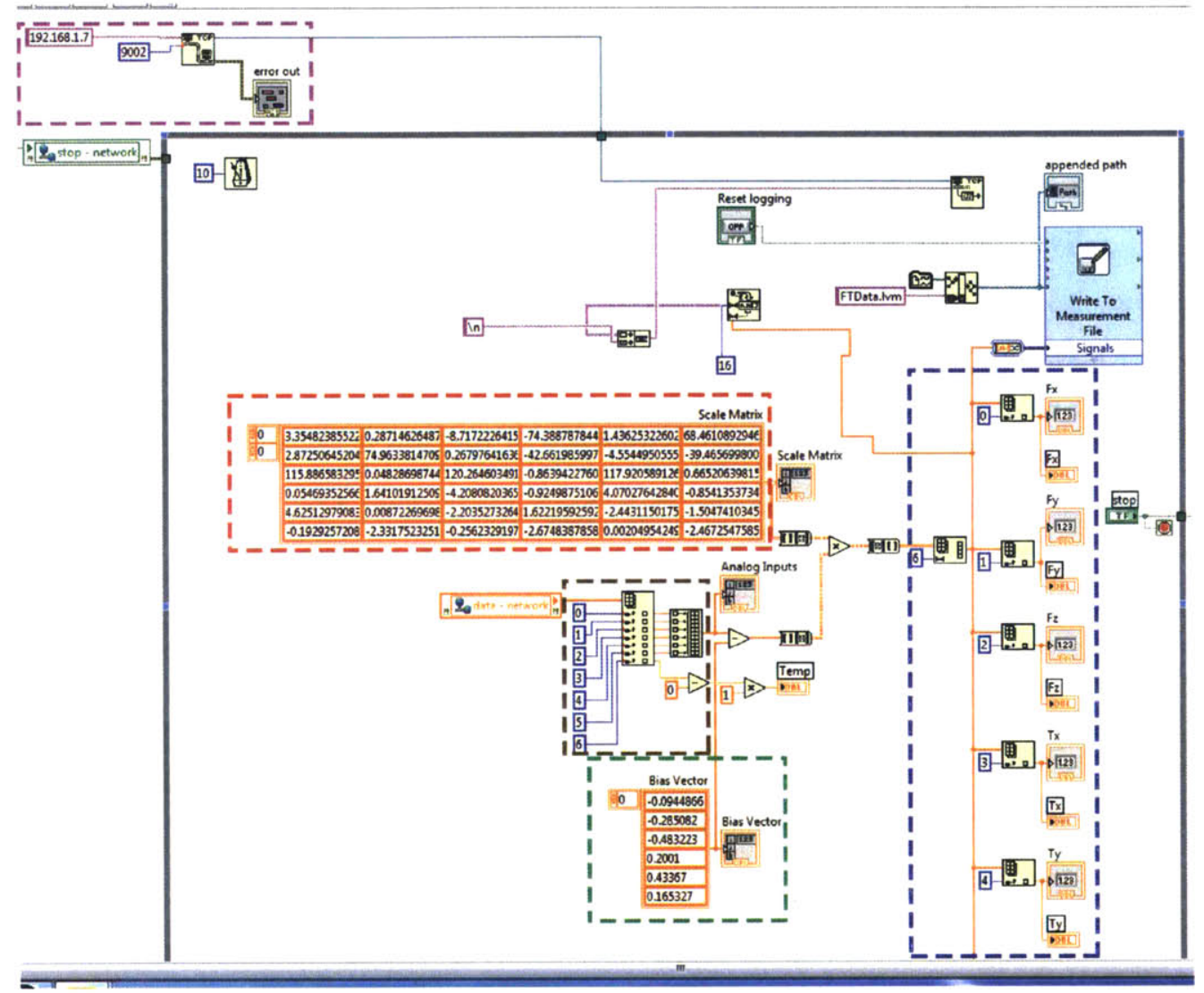

Figure 4-13: In this Labview project block diagram, manufacturer-provided calibration data (red box) and bias data (green box) is applied to incoming analog sensor data (brown box). This data is simultaneously displayed on the machine (blue box) and exported via TCP/IP (purple box).

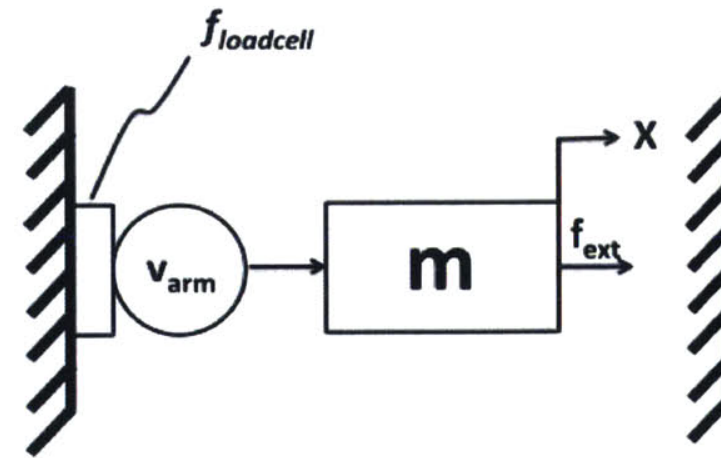

(a)

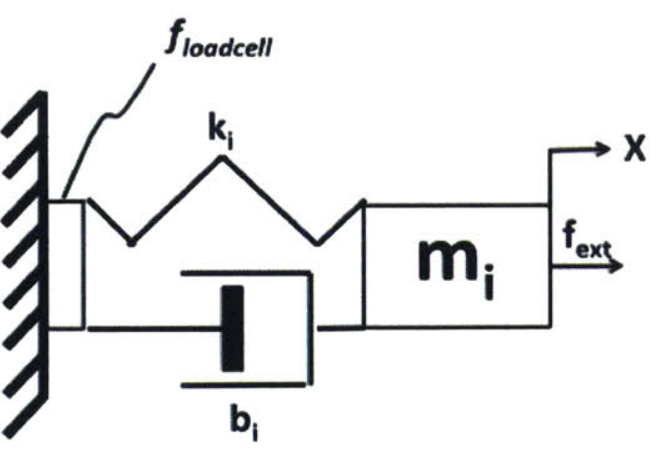

(b)

Figure 4-14: Model of linear actuator (4-14a) and model of ideal mass-spring-dashpot system (4-14b). 


\section{Developing a Transfer Function}

A very simple passive system to be achieved by the linear actuator is modeled in Figure $4-14 \mathrm{~b}$, comprising of an ideal mass, $m_{i}$, an ideal damper, $b_{i}$, and an ideal spring, $k_{i}$. In order to create a control law to have the modeled linear actuator exhibit the desired impedance characteristics modeled above, the relationship must be derived from the equations of motion.

For the actual physical system, the equation of motion is as follows:

$$
m \dot{v}=f_{e x t}-f_{l}
$$

In Laplace domain, this is:

$$
m v s=f_{e x t}-f_{l}
$$

Additionally, the equation of motion for the ideal system with desired impedance characteristics is as follows:

$$
m_{i} \ddot{x}=f_{\text {ext }}-b_{i} \dot{x}-k_{i} x
$$

Or, in Laplace domain:

$$
m_{i} v s=f_{e x t}-b_{i} v-\frac{k_{i} v}{s}
$$

Setting these two equations equal to each other by solving for $f_{\text {ext }}$ yields:

$$
m_{i} v s+b_{i} v+\frac{k_{i} v}{s}=m v s+f_{l}
$$

Multiplying through by $s$ and grouping terms produces the following, indicating a first-order relationship between input load cell reading to output velocity:

$$
\left[\left(m_{i}-m\right) s^{2}+b_{i} s+k_{i}\right] v(s)=s f_{l}(s)
$$

Assuming that the mass associated with the linear actuator is negligible, meaning that the output velocity from the model maps directly and linearly to the input velocity 
command to the actuator, the following transfer function can be obtained:

$$
\frac{v(s)}{f_{l}(s)}=\frac{s}{m_{i} s^{2}+b_{i} s+k_{i}}
$$

The transfer function demonstrates that the only dynamics relevant to this system are those of the desired impedance. This can be attributed to the linear actuator being treated as an ideal velocity source, since its internal dynamics were found to be negligible with respect to the timescales of operation, which will be expanded upon later.

\section{Developing a State-Space Model}

Writing out the state equations in their entirety yields the following Single-Input and Single-Output (SISO) system with its single input being load cell force and its single output being actuator velocity:

$$
\begin{gathered}
{\left[\begin{array}{l}
\dot{x} \\
\ddot{x}
\end{array}\right]=\left[\begin{array}{cc}
0 & 1 \\
-\frac{k_{i}}{m_{i}} & -\frac{b_{i}}{m_{i}}
\end{array}\right]\left[\begin{array}{l}
x \\
\dot{x}
\end{array}\right]+\left[\begin{array}{c}
0 \\
\frac{1}{m_{i}}
\end{array}\right] f_{l}} \\
{[\dot{x}]=\left[\begin{array}{ll}
0 & 1
\end{array}\right]\left[\begin{array}{l}
x \\
\dot{x}
\end{array}\right]+[0] f_{l}}
\end{gathered}
$$

This is a continuous-time state-space representation of the system, and must be discretized in its implementation. With a sampling time of 0.02 seconds, which is the minimum amount of time needed to gather new information from all sensors, MATLAB's c2d function discretizes the continuous time model by the zero-order hold. Given that the continuous time state-space model above has the form:

$$
\begin{aligned}
& \dot{x}(t)=A x(t)+B u(t) \\
& y(t)=C x(t)+D u(t)
\end{aligned}
$$


the discretized, zero-order hold model would have the form [23]:

$$
\begin{gathered}
x[k+1]=A_{d} x[k]+B_{d} u[k] \\
y[k]=C_{d} x[k]+D_{d} u[k]
\end{gathered}
$$

where

$$
\begin{gathered}
A_{d}=e^{A T} \\
B_{d}=A^{-1}\left(A_{d}-I\right) B \\
C_{d}=C \\
D_{d}=D
\end{gathered}
$$

and $T$ is the sample time (e.g. 0.02 seconds).

\section{Linearizing Actuator Performance}

Stiction is a common concern when using motors, and often results in a deadband of voltages at which the motor will not be set in motion. In order to test for the presence of stiction in the linear actuator, a cyclic sweep of voltage commands were sent to the actuator, covering values for $-12 \mathrm{~V}$ to $+12 \mathrm{~V}$, whose results are plotted in Figure 4-15.

The motor controller for the linear actuator takes voltage commands indicated as $\%$ of full power. The results above show a non-linear relationship between commanded voltage and actuator speed, including an asymmetric deadband at low commanded voltages. Under the desired ideal velocity source assumption, there should be a linear relationship between the commanded speed and the actual speed of the linear actuator. Fortunately, the nonlinearities exhibited in the actuator were consistent over several trials, which allowed for the nonlinearities to be simply mapped, and then compensated for when sending voltage commands to the motor controller. Results from the implemented compensation after successfully mapping the actuator response characteristics are shown in Figure 4-16: 


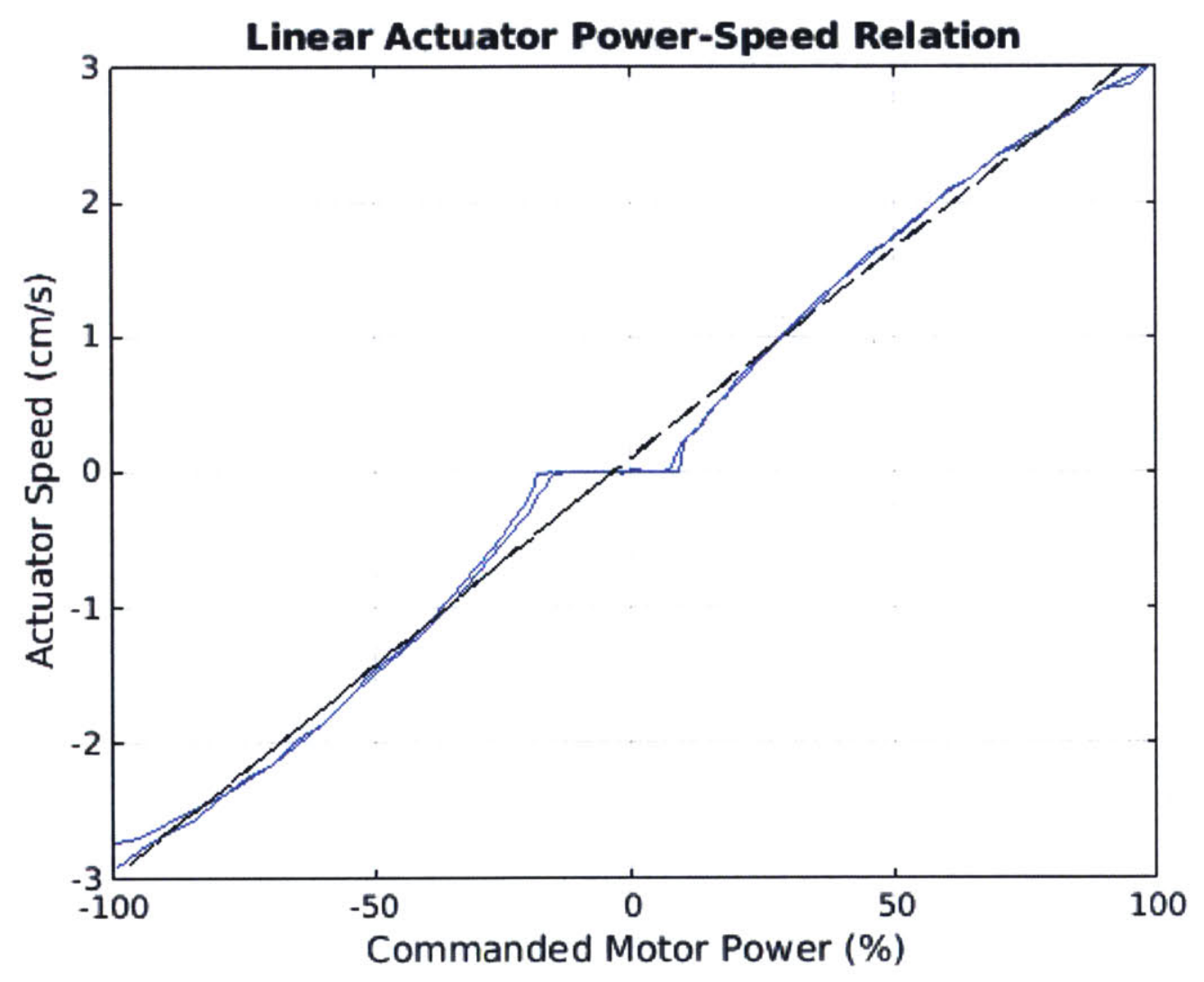

Figure 4-15: At low commanded powers, an asymmetric deadband exists that introduces a nonlinearity. Additionally, commanded power exhibits a non-linear relationship with respect to speed. 


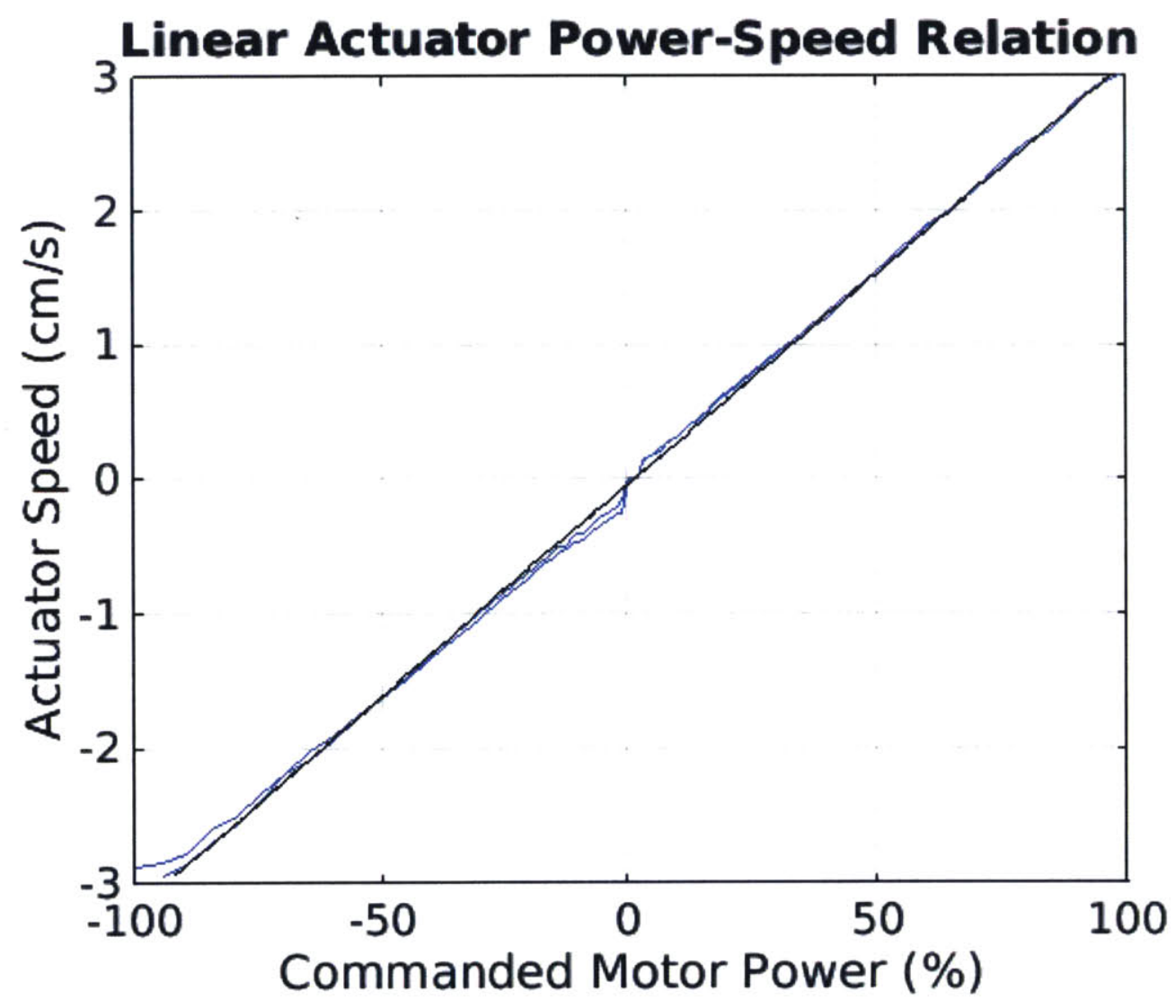

Figure 4-16: By compensating for the mappable nonlinearities that were present in the actuator's speed response, the nonlinearities are markedly reduced. 
As desired, compensating for the characterizable nonlinearities exhibited in the actuator's speed response generated an almost completely linear mapping between commanded power and the resulting speed response of the linear actuator, thus allowing for the ideal velocity source assumption.

\section{Linear Actuator Frequency Response}

In order to validate the model of the linear actuator, its frequency response was analyzed from its commanded position to its response position, resulting in the Bode plot in Figure 4-17. Position was used for the frequency response as opposed to velocity due to the fact that it was a direct measurement from the string potentiometer attached to the system. Doing so avoided otherwise noisy data from differentiating the position data. For frequencies below approximately $2 \mathrm{~Hz}(\sim 13 \mathrm{rad} / \mathrm{s})$, the system resembles a pure delay of about 0.135 seconds, confirmed across a number of driving magnitudes for consistency. Beyond this frequency, modeling the linear actuator as only having a pure delay does not hold. However, due to the fact that the system will rarely (if ever) be run at frequencies above $2 \mathrm{~Hz}$, treating the linear actuator as an ideal velocity source with a pure time delay was deemed reasonable.

\subsubsection{Creating a Control Law for the Fixed-Base in-Air Case} Passivity of Mass-Spring-Dashpot System

When analyzing a stable linear SISO system, it is passive if and only if:

$$
\forall \omega \geq 0, \operatorname{Re}[h(j \omega)] \geq 0
$$

where $h$ is the transfer function of the system. This is the same as saying that the phase shift must be between $90^{\circ}$ (Figure 4-18):

$$
\forall \omega \geq 0,|\operatorname{Arg}[h(j \omega)]| \leq \frac{\pi}{2}
$$

The concept of passivity can be easily demonstrated in the example of a simple 

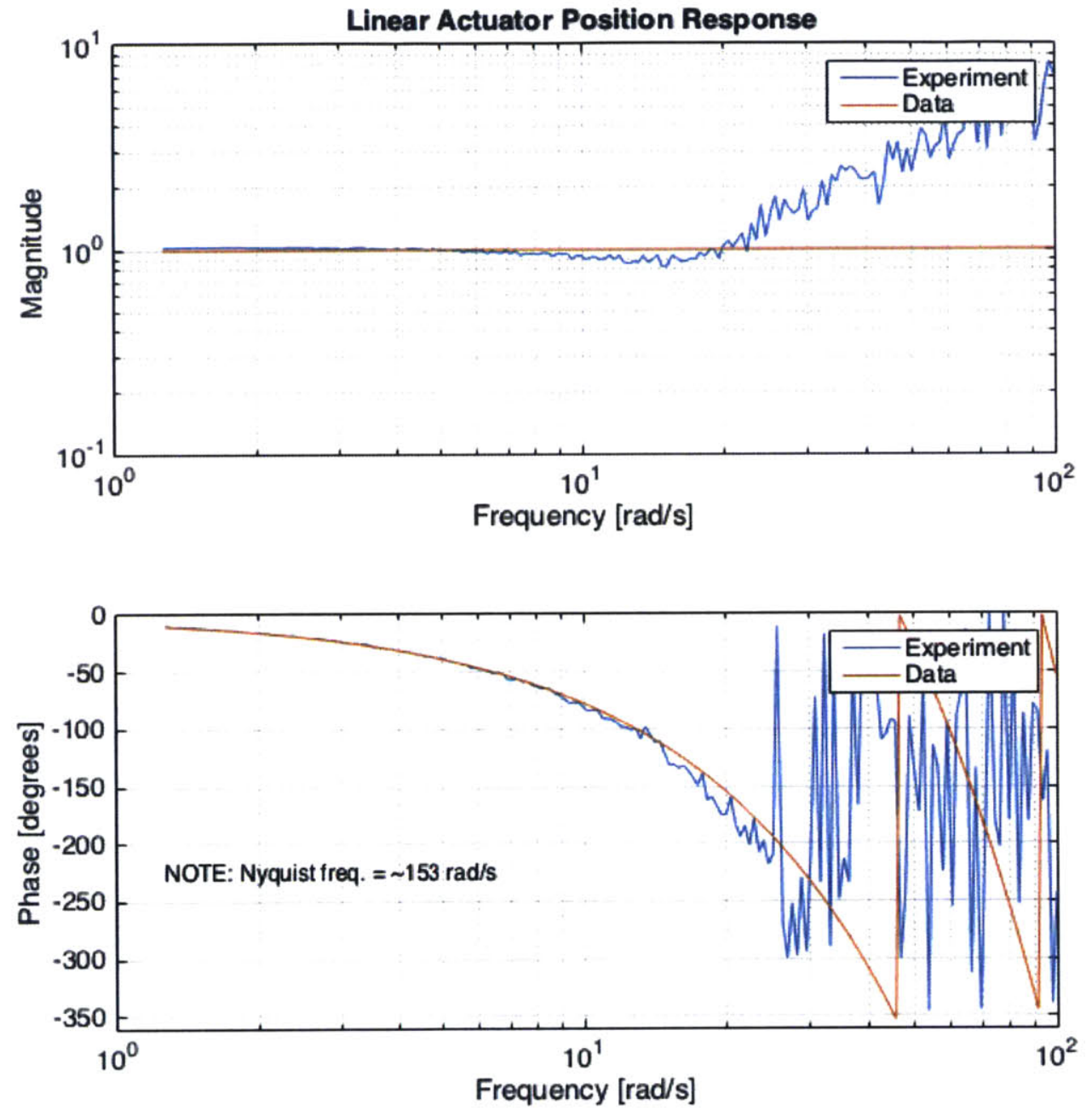

Figure 4-17: Frequency response of the linear actuator from a sinusoidal velocity command with an amplitude of $3 \mathrm{~cm} / \mathrm{s}$. The input motor power commands sent to the controller are derived from feeding the desired velocity commands through the mapped nonlinearity corrections, which ensures an approximately linear mapping between commanded motor power and actuator speed. This means that the actual power command sent to the motor controller is not that of a sinusoid, but rather a corrected value to produce a sinusoidal response in actuator speed. 


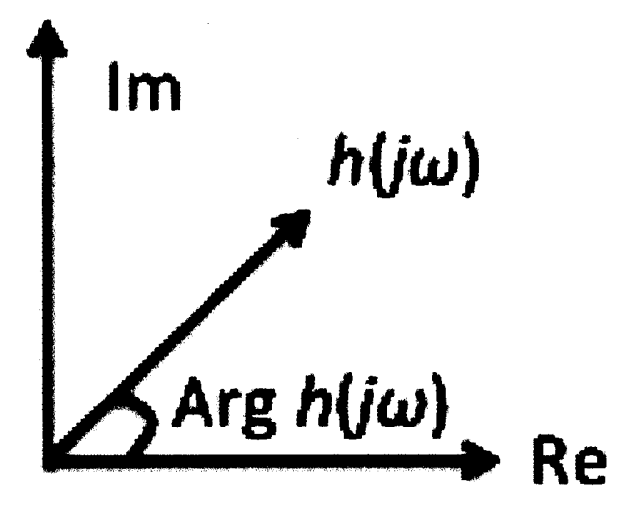

Figure 4-18: Visual representation of $|\operatorname{Arg}[h(j \omega)]|$.

mass-spring-dashpot system, as illustrated in Figure 4-19.

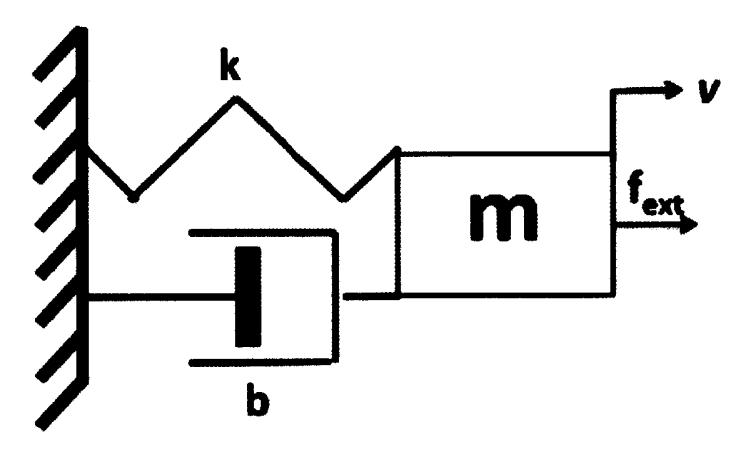

Figure 4-19: A simple mass-spring-dashpot system to be analyzed for passivity.

The transfer function for the above system is as follows, where $v$ is velocityout, and $f_{\text {ext }}$ is force $_{i n}$ :

$$
h(s)=\frac{y(s)}{u(s)}=\frac{\text { velocity }_{\text {out }}}{\text { force }_{\text {in }}}=\frac{s}{m s^{2}+b s+k}
$$

Taking any arbitrary control input between times 0 and $t$, energy when $g=0$ (i.e. passive) is [50]:

$$
V(t)=\int_{0}^{t} y(r) u(r) \mathrm{d} r=\int_{-\infty}^{\infty} y(r) u(r) \mathrm{d} r=\frac{1}{2 \pi} \int_{-\infty}^{\infty} y(j \omega) u^{*}(j \omega) \mathrm{d} \omega
$$

Note that input $u=0$ at negative times, and after $t$. Recognizing that $y(j \omega)=$ 
$h(j \omega) u(j \omega)$ yields the following:

$$
V(t)=\int_{0}^{t} y(r) u(r) \mathrm{d} r=\frac{1}{2 \pi} \int_{-\infty}^{\infty} h(j \omega)|u(j \omega)|^{2} \mathrm{~d} \omega=\frac{1}{\pi} \int_{0}^{\infty} \operatorname{Re}[h(j \omega)]|u(j \omega)|^{2} \mathrm{~d} \omega
$$

Since $h$ is a real system because of its real coefficients (mass $m$, damping $b$, and spring constant $k$ ), in the integral from - j $\omega$ to $\mathrm{j} \omega$, the real parts add while the imaginary cancel out. Therefore, the above equation points out that for $V$ to have a lower bound, $\operatorname{Re}[h(j \omega)]$ must be positive for any value of $\omega$, and thus demonstrating passivity. Plugging in the transfer function for a simple mass-spring-dashpot system yields the following:

$$
\operatorname{Re}[h(j \omega)]|u(j \omega)|^{2}=\frac{b \omega^{2}}{b^{2} \omega^{2}+\left(k-m \omega^{2}\right)^{2}} * b^{2} \omega^{2}+\left(k-m \omega^{2}\right)^{2}=b \omega^{2}
$$

What this equation shows is that as long as damping is positive, the system will always be stable; therefore creating a control law representing this mass-spring-dashpot system is a step in the right direction to pursue passive interactions between the manipulator and the decommissioning environment. However, real systems are bound by delay, which changes the passivity test in the following way:

$$
h(s)=e^{-s T} \frac{s}{m s^{2}+b s+k}
$$

Substituting $s$ for $j \omega$ yields:

$$
\operatorname{Re}[h(j \omega)]|u(j \omega)|^{2}=\operatorname{Re}\left[\frac{j \omega e^{-j \omega T}}{m(j \omega)^{2}+b j \omega+k}\right]\left|m(j \omega)^{2}+b j \omega+k\right|^{2}
$$

In order to make the denominator real, both the numerator and the denominator on the R.H.S. of equation 4.27 are multiplied by the denominator's complex conjugate:

$$
\operatorname{Re}\left[\frac{\omega \sin (\omega T)+j \omega \cos (\omega T)}{\left(k-m \omega^{2}\right)+b j \omega} \frac{\left(k-m \omega^{2}\right)-b j \omega}{\left(k-m \omega^{2}\right)-b j \omega}\right]\left|m(j \omega)^{2}+b j \omega+k\right|^{2}
$$


Simplifying and taking the real part gives the following:

$$
\left[\frac{b \omega^{2} \cos (\omega T)+k \omega \sin (\omega T)-m \omega^{3} \sin (\omega T)}{\left(k-m \omega^{2}\right)^{2}+(b \omega)^{2}}\right]\left(k-m \omega^{2}\right)^{2}+(b \omega)^{2}
$$

Finally, further simplification yields the following result:

$$
k \omega \sin (\omega T)+b \omega^{2} \cos (\omega T)-m \omega^{3} \sin (\omega T)
$$

This derivation demonstrates that, due to the addition of pure delay to the massspring-dashpot system, passivity is no longer valid for any $\omega$. However, in order to mitigate the effects of time delay, a compensator may be designed to maintain passivity for a useful range of $\omega$.

\section{Designing a Lead-Lag Controller to Mitigate Pure Time Delay Effects}

With the time delay observed in the system, the mass-spring-dashpot controller ar-

chitecture in its current state can no longer assure passivity, and it is impossible to completely remove the observed pure time delay as shown in the Bode plot in Figure 4-20. However, it is possible to design a controller to mitigate the effects of pure time delay up to a reasonable bandwidth, above which the system would not be anticipated to run. This section outlines the design criteria for a lead-lag compensator made to match the magnitude and phase of up to the ideal system's damped natural frequency, while being sure to maintain a response magnitude at low frequencies in order to ameliorate steady-state error.

A lead controller has the following form (Figure 4-21):

$$
H_{\text {lead }}(s)=K_{\text {lead }} \frac{\alpha_{\text {lead }} \tau_{\text {lead }} s+1}{\tau_{\text {lead }} s+1}
$$

The goal of this lead compensator in the lead-lag controller is to match the magnitude and phase of the ideal system in the time delayed system. A method to do so is by choosing $\tau_{\text {lead }}$ such that the maximum phase of the compensator coincides with the 

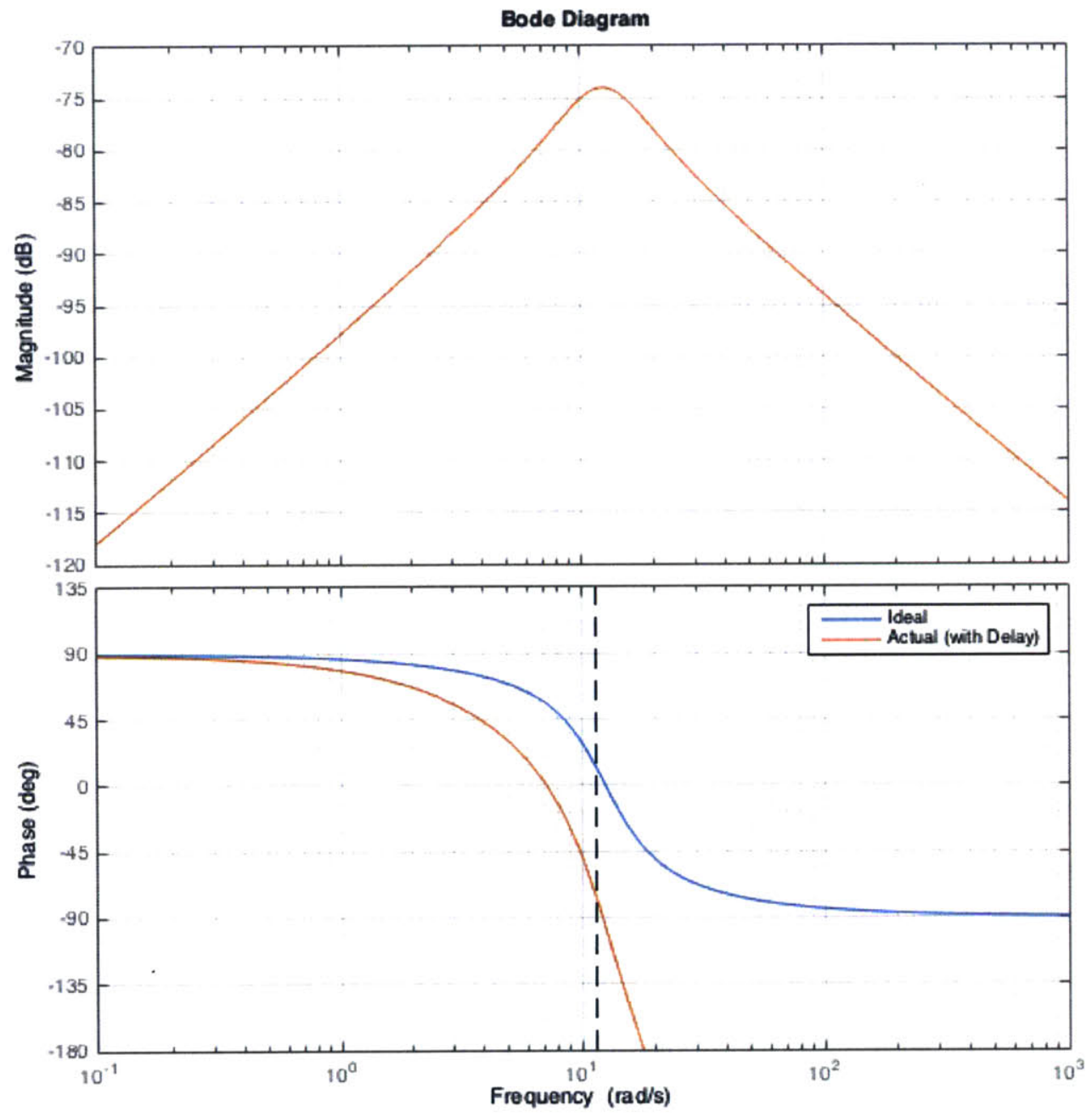

Figure 4-20: The above Bode plot shows the ideal system both with and without delay. Without the time delay, phase is maintained with $\pm 90^{\circ}$. However, the time delay introduces a linear increase in phase lag as a function of frequency. 


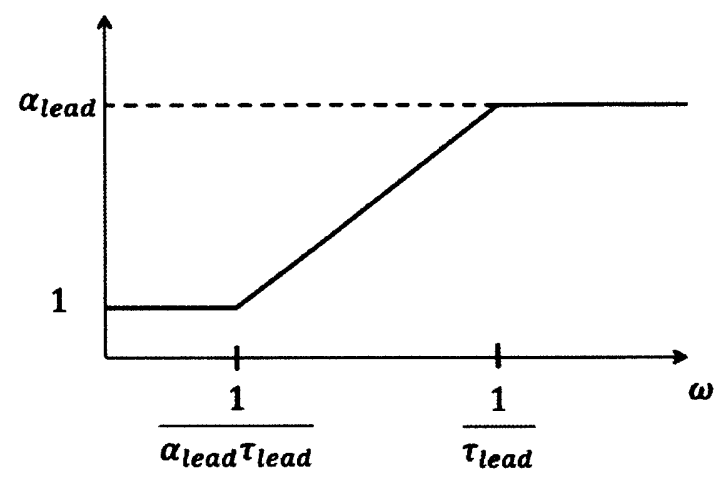

(a)

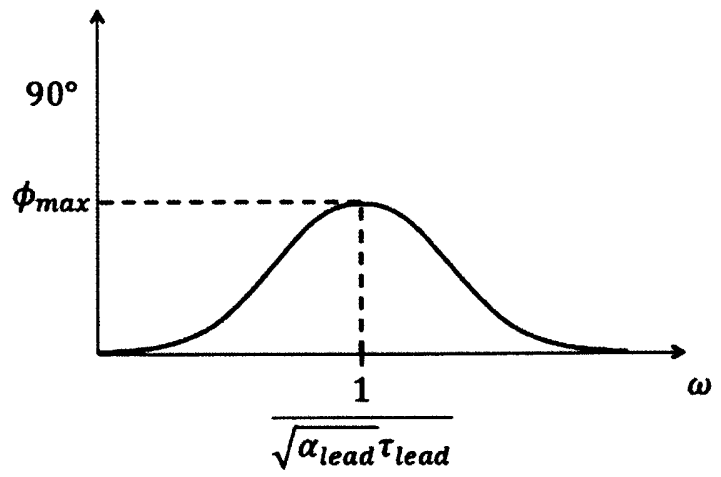

(b)

Figure 4-21: Magnitude (4-21a) and phase (4-21b) plot of a generic lead compensator.

damped natural frequency of the system. This point occurs at:

$$
\tau_{\text {lead }}=\frac{1}{\sqrt{\alpha_{l e a d}} \omega_{d}}
$$

Next, a value for $\alpha_{\text {lead }}$ must be chosen such that the phase lost from the time delay is added back in. The amount of phase that needs to be added back can be found by using the following equation for peak phase in a lead compensator:

$$
\phi_{\text {delay }}=\sin ^{-1}\left(\frac{\alpha_{\text {lead }}-1}{\alpha_{\text {lead }}+1}\right)
$$

Finally, a value for $K_{\text {lead }}$ must be chosen such that the magnitude at the damped natural frequency is preserved:

$$
K_{\text {lead }}=\frac{1}{\sqrt{\alpha_{\text {lead }}}}
$$

A lag controller has the following form (Figure 4-22):

$$
H_{l a g}(s)=K_{l a g} \frac{\tau_{l a g} s+1}{\alpha_{l a g} \tau_{l a g} s+1}
$$

The goal of this lag compensator in the lead-lag controller is to restore the lowfrequency magnitude offset created by implementing the lead compensator. A method to do so is to set $\tau_{l a g}$ at the minimum distance away from the lead component that 
will not affect it. A reasonable location for this point occurs at:

$$
\tau_{\text {lag }}=\frac{1}{\sqrt{\alpha_{\text {lead }}} \omega_{d} * 10^{-2}}
$$

Next, a value for $K_{l a g}$ must be chosen such that the low frequency gain counteracts the magnitude loss from the lead component:

$$
K_{l a g}=\sqrt{\alpha_{l e a d}}
$$

Finally, a value for $\alpha_{\text {lag }}$ must be chosen to ensure that the high frequency gain is unity:

$$
\alpha_{l a g}=\sqrt{\alpha_{l e a d}}
$$

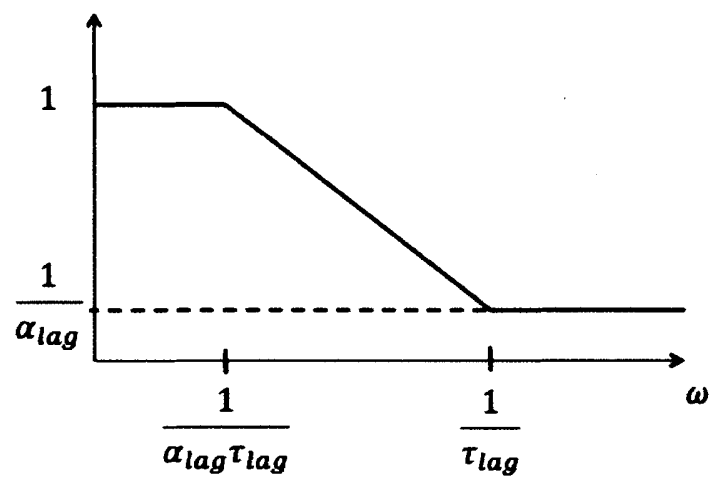

(a)

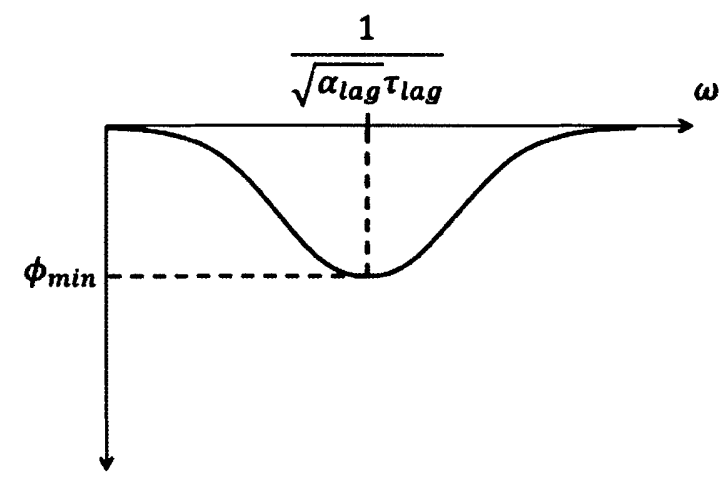

(b)

Figure 4-22: Magnitude (4-22a) and phase (4-22b) plot of a generic lag compensator.

A Bode plot of the combined lead-lag compensator along with the individual lead and lag components is shown in Figure 4-23. Key features of this Bode plot include unity gain for low frequencies, and zero phase lag for both low and high frequencies.

The plot in Figure 4-24 demonstrates the influence of the designed compensator on the time-delayed system. The lead component works to match the ideal magnitude and phase at its damped natural frequency, while the lag component works to restore the ideal magnitude at low frequencies. A step response is also plotted of the ideal system, along with time-delayed system with and without compensation. Although 


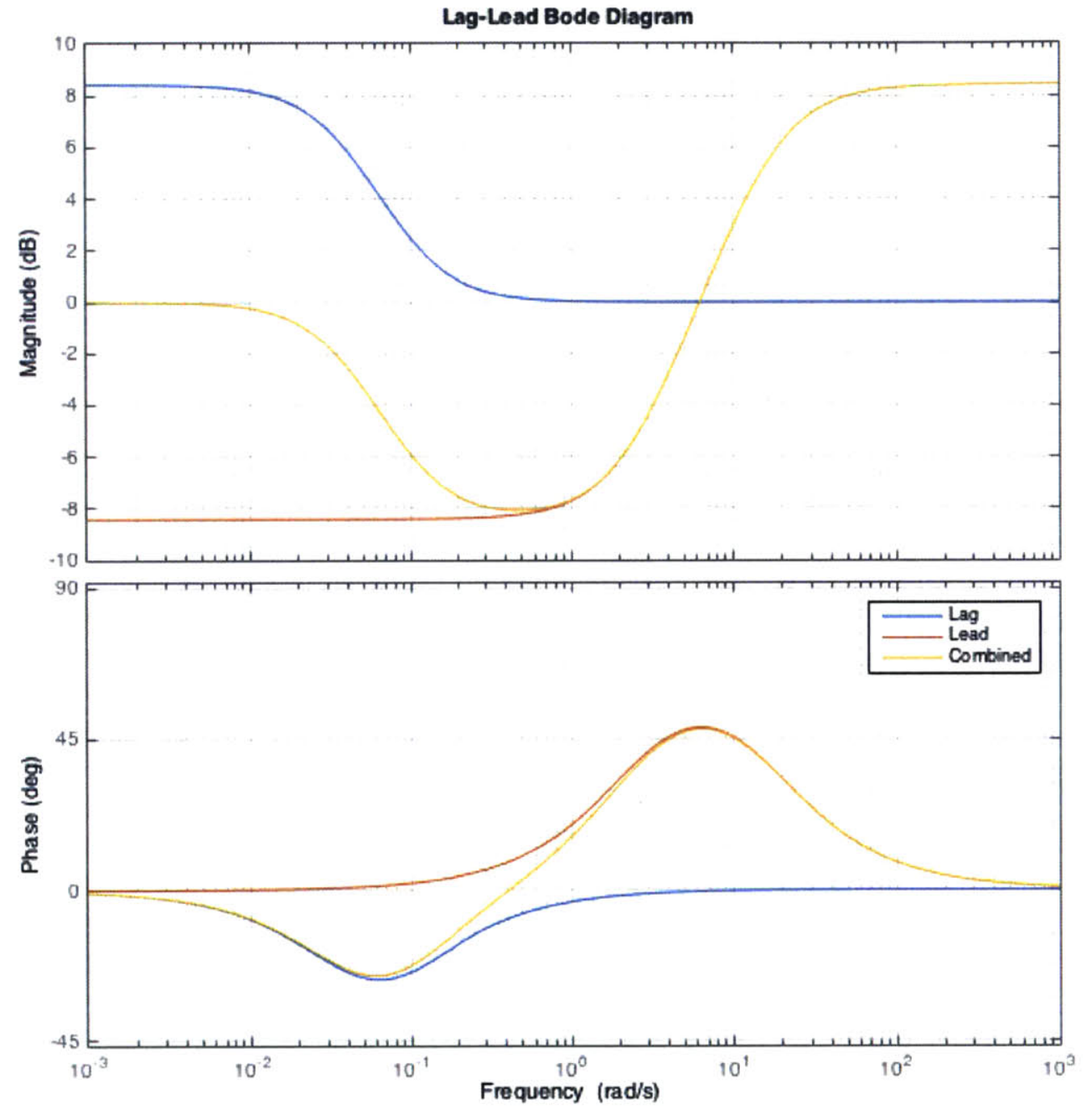

Figure 4-23: The magnitude and phase Bode plot shows the individual lead (red) and lag (blue) component, along with the combined lead-lag compensator (yellow). 
plot shows a slight overshoot by the compensated system relatively to the ideal system, the compensated system quickly converges on the correct magnitude and phase of its ideal counterpart.
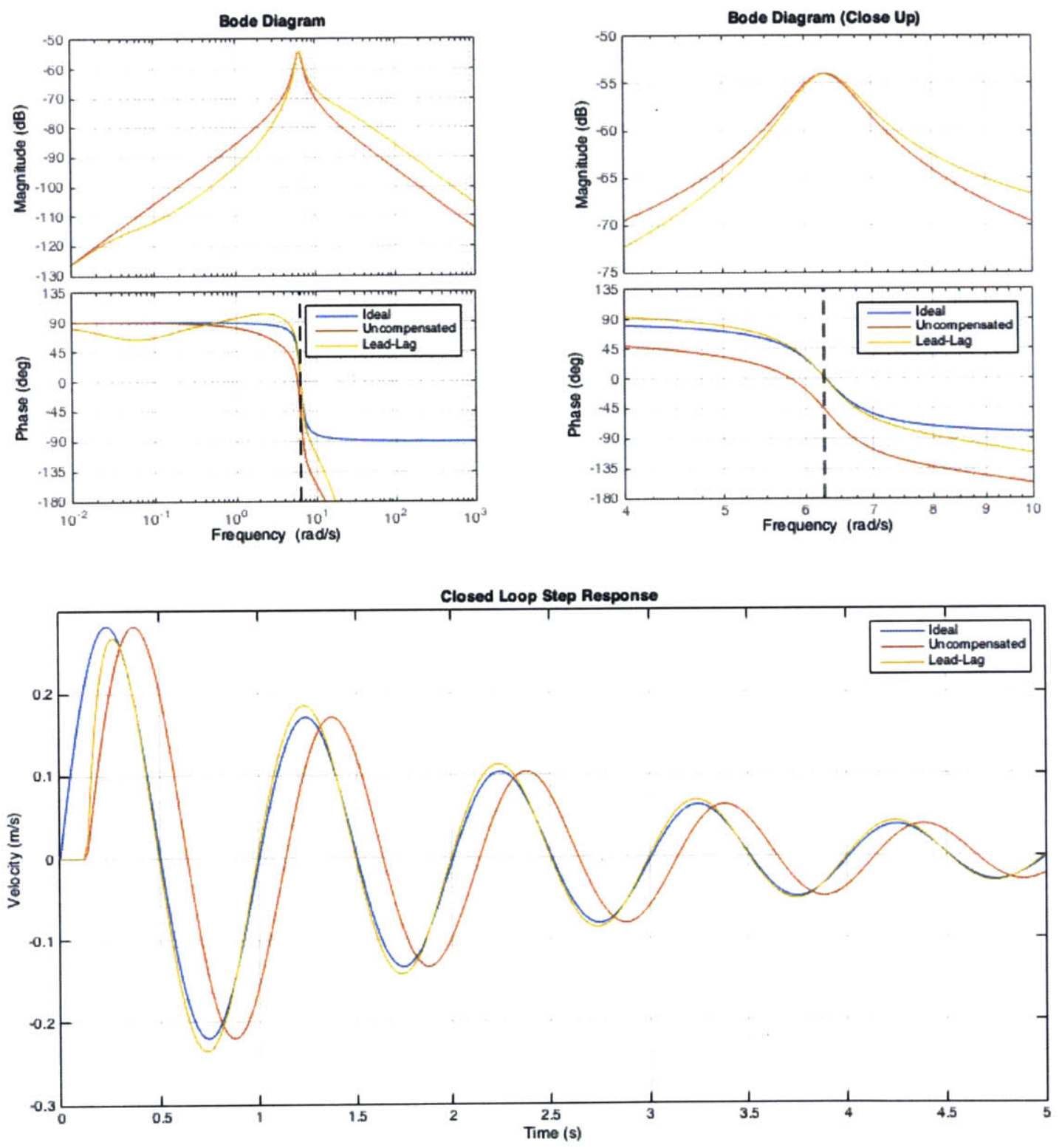

Figure 4-24: Bode plots of the ideal, uncompensated, and compensated systems are shown (top-left and top-right), along with corresponding step responses for each (bottomt). 


\subsubsection{Experimental Results}

Research in stable robotic interactions has indicated that one of the worst environments with which a linear system can interact is one with solely a set of springs and masses [18]; this environment was ultimately decided as the scenario in which to test the passivity controller (Figure 4-25). The end effector was coupled with a compression spring, which collided with a suspended mass of approximately $13 \mathrm{~kg}$, and had a combined natural frequency of approximately $1 \mathrm{~Hz}$. The controller was run based on the state-space model using the measured load cell reading as the input vector and the end effector velocity as the output vector, and incorporated the passive impedance characteristics of the desired system. Given appropriate initial conditions, the model predicted the state vector at each time step, which was iterated and modified given the new force input data at each time step.

With respect to analyzing the vehicle's response to a compliant-tip collision against a hanging mass, the following results are summarized in Figure 4-26.

The controller demonstrated that it was able to transform a non-backdrivable, position-controlled actuator with delay into a stable system with user-defined impedance characteristics, resulting in a reduction in peak impact force and total settling time by approximately $24 \%$ and $91 \%$, respectively. However, it should be noted that in the presence of delay, a peak force reduction is only possible with enough mechanical compliance, such as with the spring tip on the end effector. This provides a buffer for the controller to react to any intentional or unforeseen collisions before the entire force of impact has been imparted onto the vehicle. This is particularly valuable for autonomous decommissioning, since it provides a level of safety for the vehicle against any potential damage from the shock of impact. Given the drive to remove support vessels as much as possible from the process, impact damage to an autonomous decommissioning vehicle would be especially detrimental without the presence of a nearby support vessel to service the vehicle.

A sharp reduction in settling time suggests a smaller influence by external disturbances, such as underwater currents. Passivity's guarantee for stability only holds 


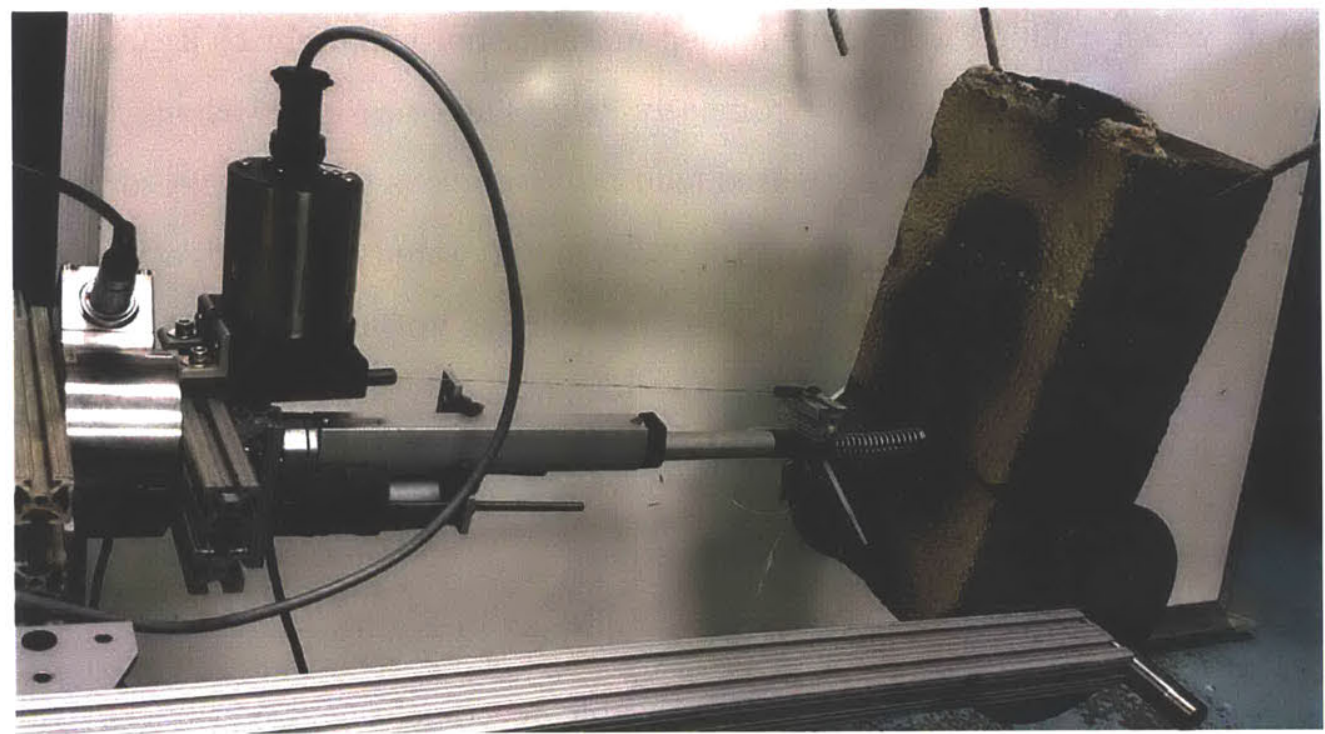

(a)

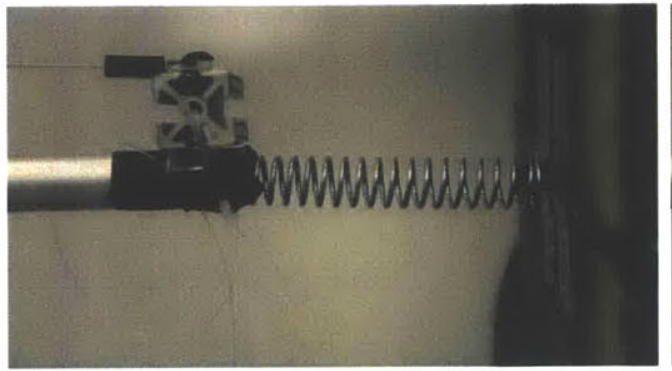

(b)

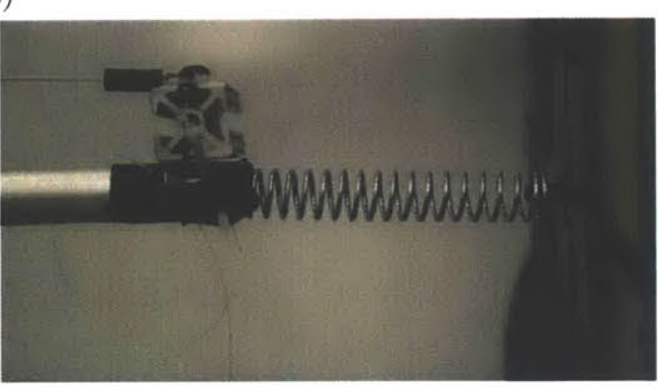

(c)

Figure 4-25: A comparison was measured between actuator response to a complianttip collision with a hanging mass when the controller is turned on or off. 

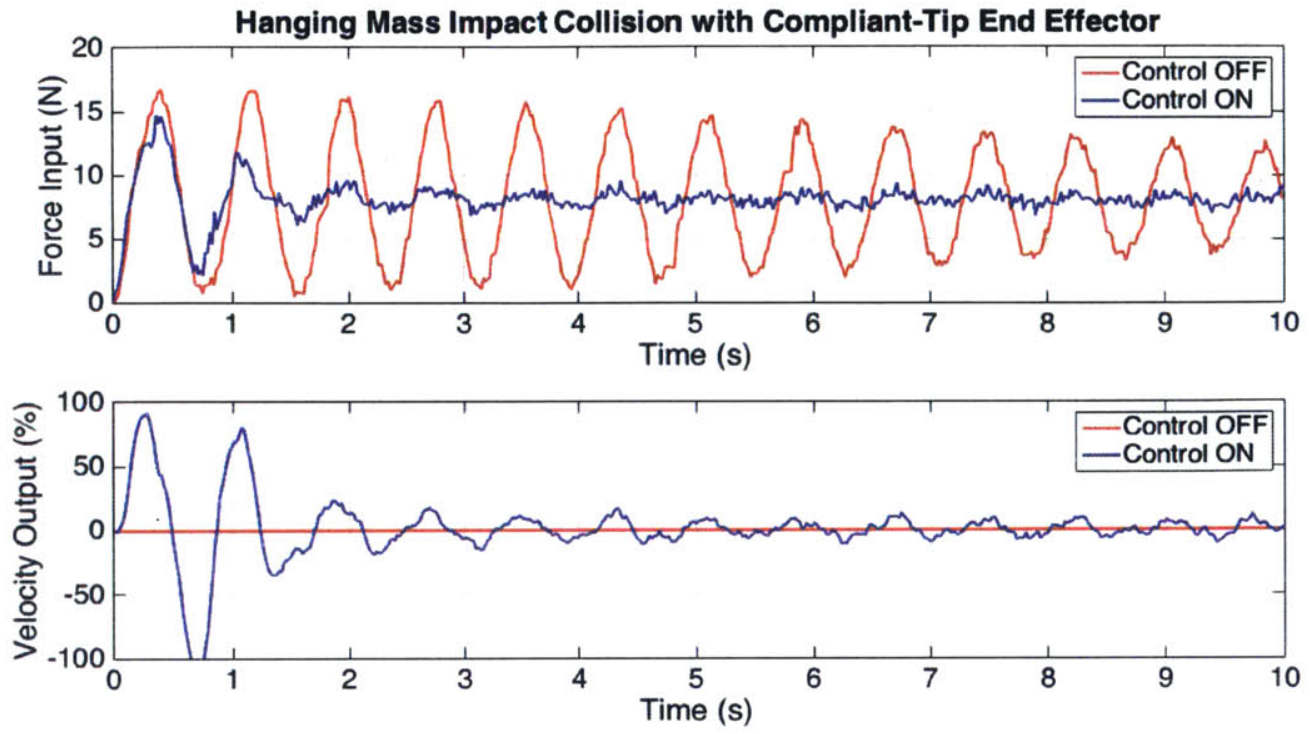

Figure 4-26: Comparisons of force inputs and velocity output of the actuator when the controller is either on or off. The mass, $m_{i}$ is $500 \mathrm{~kg}$, the damping, $b_{i}$ is $500 \mathrm{~kg} / \mathrm{s}$, and the stiffness, $k_{i}$ is $30,843 \mathrm{~N} / \mathrm{m}$ in order to achieve a natural frequency of $1.25 \mathrm{~Hz}$.

for interactions with a passive environment, which remains valid for the collision of a vehicle against a subsea structure. However, currents interacting with the vehicle and/or structure introduce energy into the system. Accounting for this when designing the controller would prove to be difficult due to the computation necessary to best model the unpredictability of currents, so at the very least, a reduction in settling time provides an ability to minimize the unpredictable disturbances to any ongoing contact tasks performed by the decommissioning vehicle.

These results have relevant applications towards the industry's already heavy usage of position-controlled robotic manipulators, and provide a strong foundation for expansion to a raft-robot system with multi-dimensional impedance. 


\subsection{Analysis of the Vehicle-Manipulator System Free- Floating in Water}

\subsubsection{Modeling the Raft and Linear Actuator}

The next step from the manipulator on a fixed base presented in Section 4.4.1 is to remove the fixed-case constraint and include the dynamics of the raft and its thrusters. As previously presented in 4-2b, and now shown in Figure 4-27, a model of the full system is comprised of the mass of the raft, $m$, with its associated damping, $b$, the thruster force, $f_{\text {thruster }}$, the velocity of the manipulator, $v_{a r m}$, the mass of the manipulator, $m_{2}$, with its associated damping, $b_{2}$, and external force, $f_{e x t}$, acting upon it.

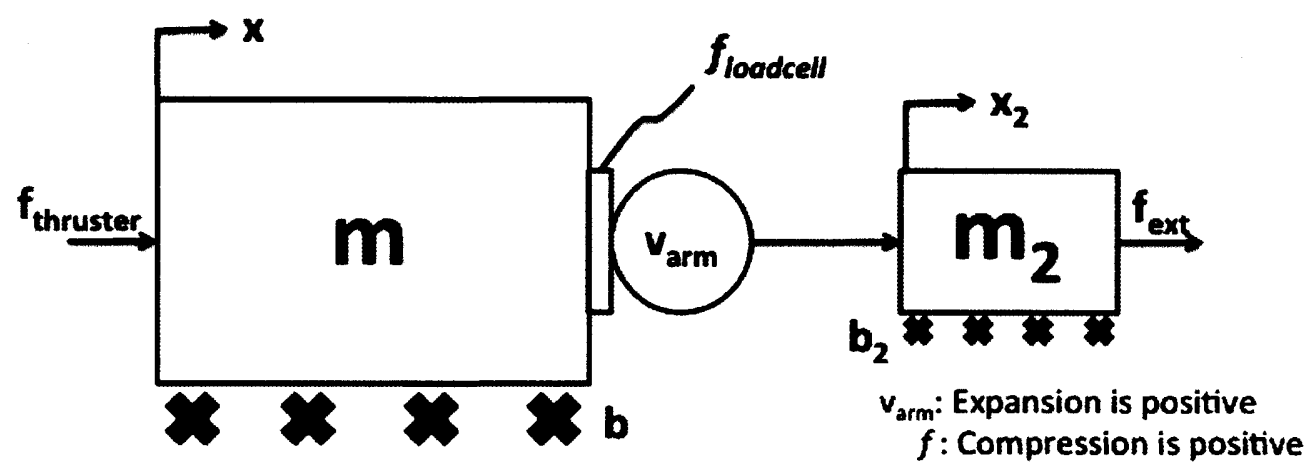

Figure 4-27: A model of the physical system shows the base of the manipulator attached to a free-floating raft. The inclusion of the free-floating raft adds another sensor (raft position, $x$ ) and another actuator (ROV thruster, $f_{\text {thruster }}$ ), making this a Multiple-Input and Multiple-Output (MIMO) system. It should be noted that this model does not share the same notation as the previously presented fixed raft case.

The target passive system is pictured in Figure 4-28, and represents the physical system as two masses in a series of springs and dampers.

The equations of motion for the physical system are as follows:

$$
\begin{gathered}
m \ddot{x}=f_{t}-f_{l}-b \dot{x} \\
m_{2} \ddot{x}_{2}=f_{l}+f_{e}-b_{2} \dot{x}_{2}
\end{gathered}
$$




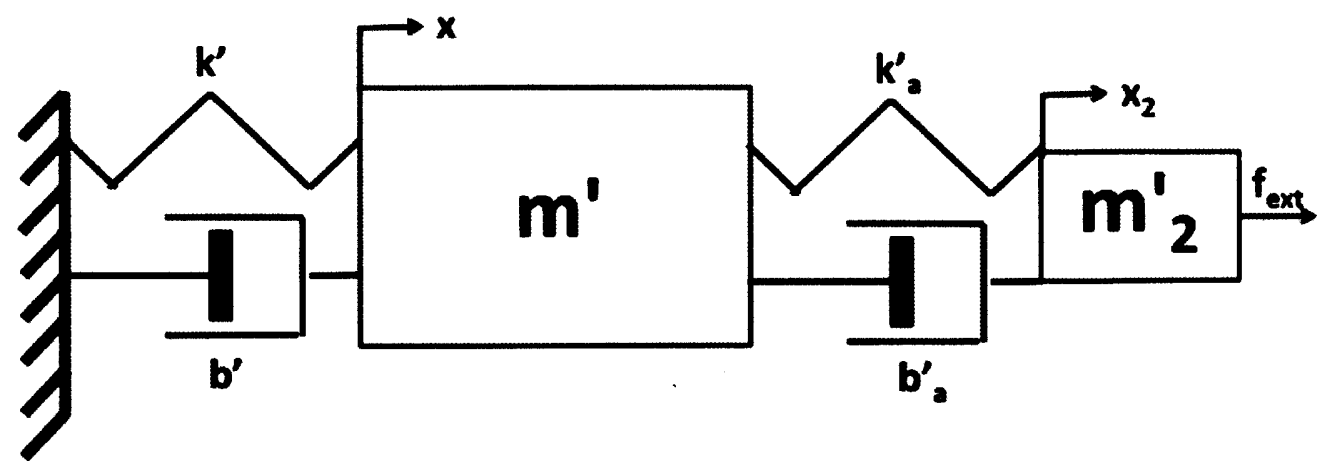

Figure 4-28: A model of the ideal system replaces the actuators with passive components comprised of springs and dashpots.

$$
x_{a}=x_{2}-x
$$

On the other hand, the equations for the ideal system are as follows:

$$
\begin{gathered}
m^{\prime} \ddot{x}=b_{a}^{\prime} \dot{x}_{a}+k_{a}^{\prime} x_{a}-b^{\prime} \dot{x}+k^{\prime} x \\
m_{2}^{\prime} \ddot{x}_{2}=f_{e}-b_{a}^{\prime} \dot{x}_{a}-k_{a}^{\prime} x_{a} \\
x_{a}=x_{2}-x
\end{gathered}
$$

Using the two sets of equations of motion for the physical system and ideal system, the actual physical system and the ideal system can be related to each other. Using equations 4.39 and 4.42 , the following equations show the relationship between the input load cell reading, $f_{l}$, to the two outputs, thruster force $f_{t}$ and arm velocity $v_{a}$.

Rearranging equation 4.39 from the real system and transforming to Laplace domain provides the following:

$$
\frac{f_{t}(s)-f_{l}(s)}{m s^{2}+b s}=x(s)
$$

Rearranging equation 4.42 from the ideal system and transforming to Laplace domain yields:

$$
\frac{b_{a}^{\prime} s+k_{a}}{m^{\prime} s^{2}+b^{\prime} s+k^{\prime}} x_{a}(s)=x(s)
$$


Setting these two equations equal to each other gives:

$$
f_{l}(s)=f_{t}(s)-\frac{\left(b_{a}^{\prime} s+k_{a}\right)\left(m s^{2}+b s\right)}{m^{\prime} s^{2}+b^{\prime} s+k^{\prime}} x_{a}(s)
$$

Using equations 4.40 and 4.43 , the following equations show the relationship between the vehicle position, $x$, to the two outputs, thruster force $f_{t}$ and arm velocity $v_{a}$.

Rearranging equation 4.40 from the real system and transforming to Laplace domain provides the following:

$$
\left[\left(m+m_{2}\right) s^{2}+\left(b+b_{2}\right) s\right] x(s)+\left[m_{2} s^{2}+b_{2} s\right] x_{a}(s)-f_{t}(s)=f_{e}(s)
$$

Rearranging equation 4.43 from the ideal system and transforming to Laplace domain yields:

$$
\left[m_{2}^{\prime} s^{2}+b_{a}^{\prime} s\right] x_{a}(s)+\left[m_{2}^{\prime} s^{2}\right] x(s)=f_{e}(s)
$$

Setting these two equations equal to each other gives:

$$
x(s)=\frac{1}{\left(\left(m_{2}-m_{2}^{\prime}\right)+m\right) s^{2}+\left(b+b_{2}\right) s} f_{t}(s)+\frac{\left(m_{2}^{\prime}-m_{2}\right) s^{2}+\left(b_{a}^{\prime}-b_{2}\right) s+k_{a}}{\left(\left(m_{2}-m_{2}^{\prime}\right)+m\right) s^{2}+\left(b+b_{2}\right) s} x_{a}(s)
$$

These two derived equations can be combined to create a transfer matrix for this MIMO system, which can be used in the future for controller design for free-flight experiments with the vehicle:

$$
\left[\begin{array}{l}
x \\
f_{l}
\end{array}\right]=\left[\begin{array}{cc}
\frac{\left(m_{2}^{\prime}-m_{2}\right) s^{2}+\left(b_{a}^{\prime}-b_{2}\right) s+k_{a}}{\left(\left(m_{2}-m_{2}^{\prime}\right)+m\right) s^{2}+\left(b+b_{2}\right) s} & \frac{1}{\left(\left(m_{2}-m_{2}^{\prime}\right)+m\right) s^{2}+\left(b+b_{2}\right) s} \\
-\frac{\left(b_{a}^{\prime} s+k_{a}\right)\left(m s^{2}+b s\right)}{m^{\prime} s^{2}+b^{\prime} s+k^{\prime}} & \frac{m^{\prime} s^{2}+b^{\prime} s+k^{\prime}}{m^{\prime} s^{2}+b^{\prime} s+k^{\prime}}
\end{array}\right]\left[\begin{array}{c}
x_{a} \\
f_{t}
\end{array}\right]
$$

\subsubsection{Linearizing Thrusters}

In preparation for future free-floating contact experiments, the ROV thrusters' steadystate dynamics were modeled and linearized. Figure 4-29 shows a characteristic square relationship between motor voltage and thrust production [53]. The asymmetric design of the thruster generates asymmetric responses between forward and reverse 
thrust, but both are still indicative of a square relationship. This response proved to be repeatable, which allowed for a corrective mapping of its non-linear response that is presented in Figure 4-30.

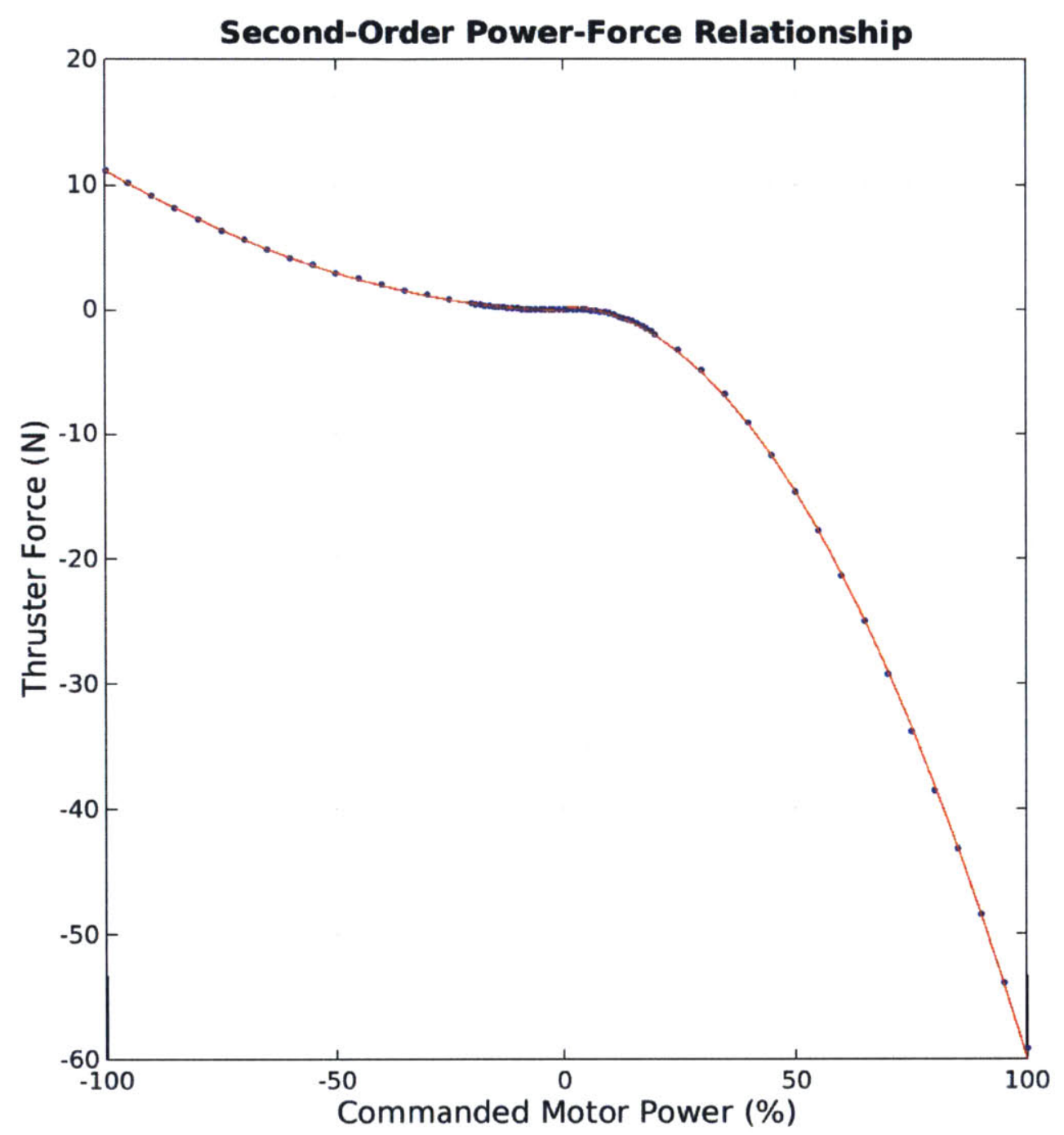

Figure 4-29: Fitting the static thruster data shows a typical square relationship between power and thrust.

\subsubsection{Thruster Frequency Response}

If there are any significant transient thruster dynamics that exist at the desired operating bandwidth, these characteristics must be incorporated back into the model. 


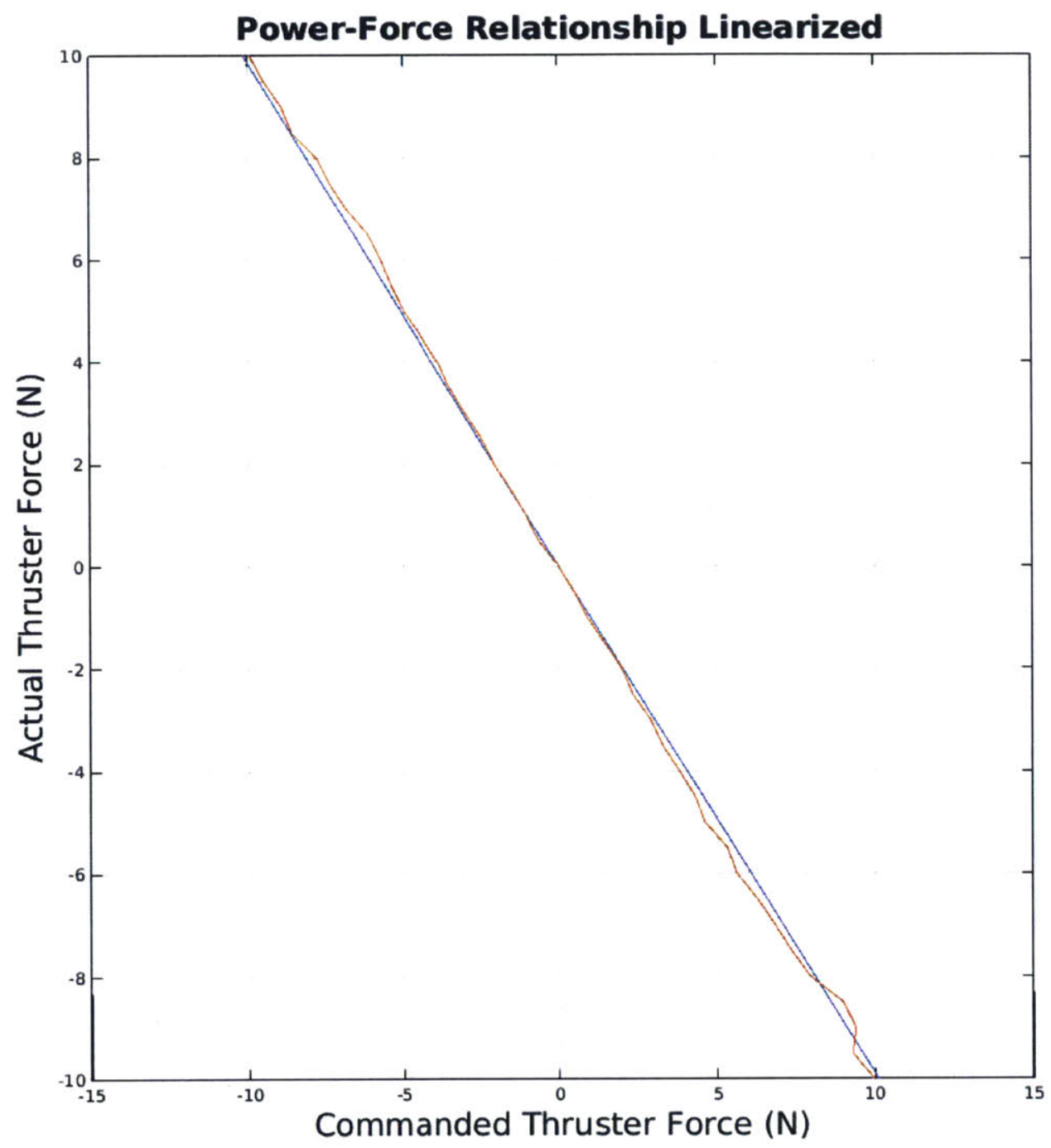

Figure 4-30: Since the thrust response to input power proved to be repeatable, it allowed for a corrective mapping of its non-linear response. 
In order to test for this, the end effector of the raft was fixed to the wall of the testing tank, so that any force from the thrusters would translate to only axial loads registered on the load cell, and not movement of the raft. Figure 4-31 presents the thruster force frequency response data collected from the motors attached to the fixed raft in water. Due to the uncaptured nonlinearities in the thruster's dynamics, the Bode plot expresses a steady rise in magnitude given a steady increase in phase lag. This may be in part due to the fact that the mapping of nonlinearities used to actuate thrust from power was based on steady-state results. Therefore, as the frequency at which the system was run increased to create the Bode plot, uncharacterized transient state relationships between input power and thruster force became more prominent. These irregular results indicate that more work must be done in understanding the nonlinear dynamics in the Inuktun ROV Thrusters before any assumptions of an LTI system can be made.

\subsection{Conclusion}

Impedance control has found its value in other industries, whether it is programmable impedance, impedance inherent in mechanical design, or both. These industries include the medical industry, where interactions with humans can be extremely sensitive to force [52]. However, unlike many of these industries, the environments in the offshore industry are unforgivingly harsh, which dictate the design of manipulators made to be mounted on rugged vehicles built to interact with massive steel structures [56]. As such, the industry standard in these manipulators drives for strength, and therefore incorporates little backdrivability into their designs. These challenging environments also demand the dexterity and interactive capabilities of a human, which over time has made the potential role of impedance control robotics more prominent.

Successfully operating a non-backdrivable, high-gear reduction linear actuator to have a passive impedance is just the first step in setting the framework to create robotic interactions that can simulate human muscle movements, and ultimately eliminate the risk of life from these unfortunately difficult and necessary tasks. Future 

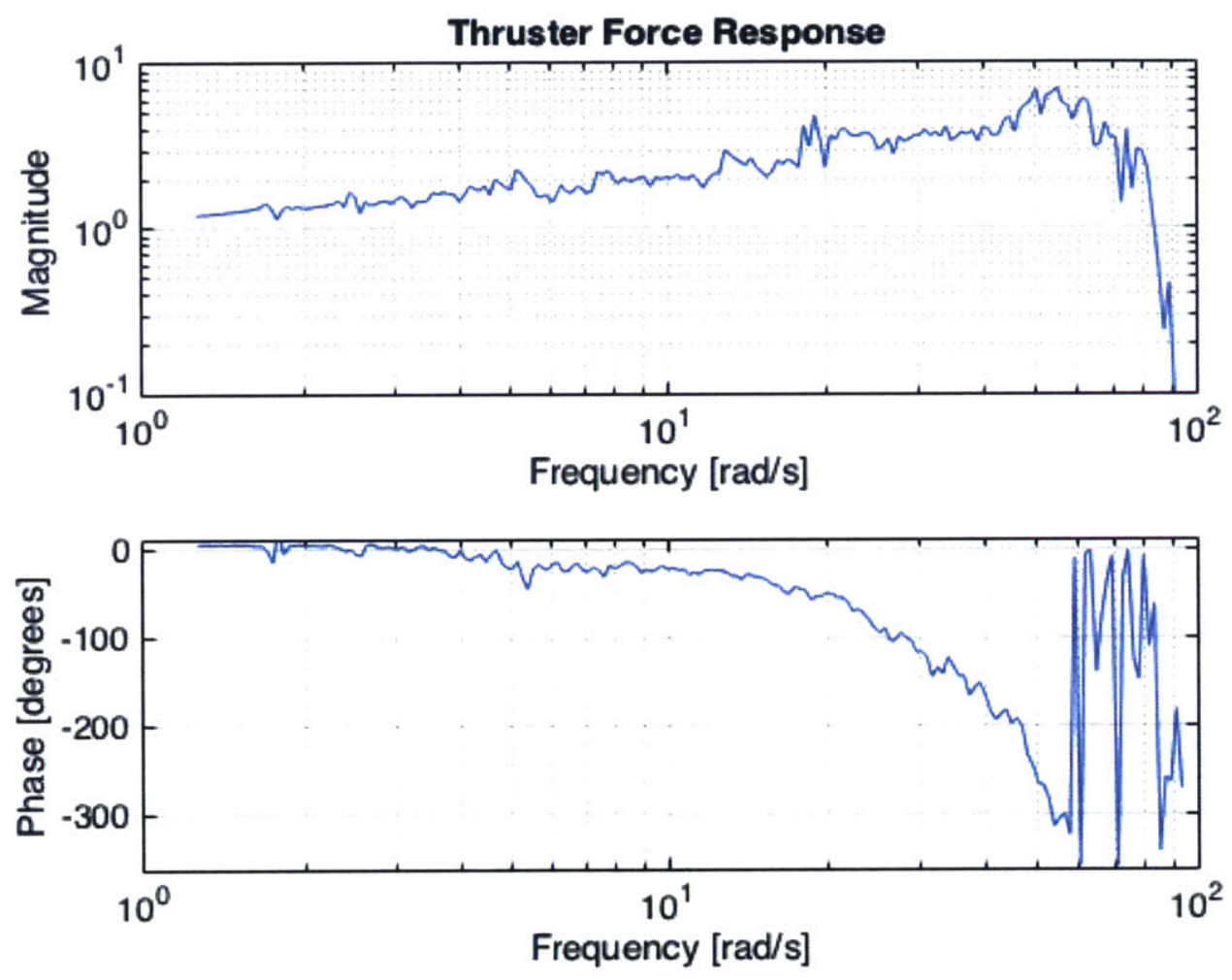

Figure 4-31: Similar to the linear actuators, the thrusters exhibit time delay characteristics. However, irregular traits, such as a steady rise in magnitude given a steady increase in phase lag, indicate that more work must be done in understanding the nonlinear dynamics in the thrusters. This frequency response is from a sinusoidal thrust command with an amplitude of $10 \mathrm{~N}$. The input motor power commands sent to the controller are derived from feeding the desired thrust commands through the mapped nonlinearity corrections, which ensures an approximately linear mapping between commanded motor power and steady-state thruster force. This means that the actual power command sent to the motor controller is not that of a sinusoid, but rather a corrected value to produce a sinusoidal response in steady-state thruster force. 
work must still be done with the current model, determining impedance ranges that maintain stability, and how that varies with different linear actuators and computational methods. The analysis must also be broadened to include the entire free-floating raft-robot system, and the concepts mastered from the linear actuator analysis provide strong ties to understanding how to have this system interact passively in a desirable way.

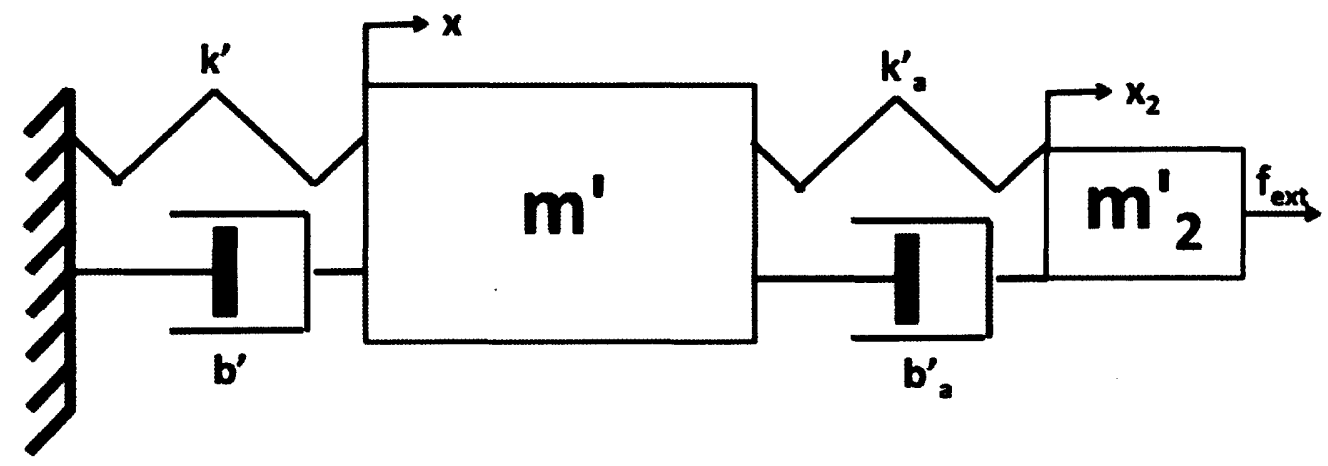

Figure 4-32: Raft-robot setup modeled as a passive system.

In the model in Figure 4-32, for very small values of $m_{2}$, this system would parallel a station-keeping body with a tactile end effector, which is extremely important given that station-keeping is necessary for dangerous and cluttered environments, while forms of compliance are necessary for uncertain currents and motions from an underwater environment.

As time progresses, the environments in which decommissioning is necessary is becoming increasingly severe, and approaching the limits of what is possible to achieve with only divers and ROVs alone. By analyzing the ways in which ties can be drawn between human and robotic interaction with uncertain environments, the ceiling of autonomous interactive capabilities can be lifted, enabling a future where no human lives have to be risked merely for their sense of touch. 


\section{Chapter 5}

\section{Conclusions}

This thesis discusses Autonomous Underwater Vehicles (AUVs) as a solution to conduct cost-effective and safe decommissioning, lessening dependence on ROVs and divers, while still adhering the oil and gas industry's strict environmental standards.

\subsection{Summary of Work}

Chapter 1 introduces the problems with the current state of decommissioning; the drawbacks of the continued usage of ROVs and divers are addressed, and the use of autonomy is proposed. AUVs already play a large role in inspection tasks for the oil and gas industry, and the time has come to utilize their advantages for subsea intervention.

Chapter 2 suggests using 3D environment mapping for autonomous navigation around subsea structures. Common methods for offshore navigation are generally inadequate, so the Iterative Closest Point method is implemented to correct INS integration drift, using a 3D sonar reference map of an offshore platform.

Chapter 3 delves into AUV bracing onto a subsea structure with a complex geometry. Current methods for free-flight interaction do not address limited effectiveness of cameras and laser range finders, or the presence of unpredictable currents. A design is proposed to simplify bracing onto a structure from free-flight. A prototype was fabricated, and its functionality verified. 
Chapter 4 discusses autonomous manipulation of subsea structures from a freeflight vehicle when bracing options are not given. Passivity is proposed as a way to promote robust stability in interactions with the decommissioning environment. A controller is designed to transform a non-backdrivable, position-controlled actuator with delay into a passive system with user-defined impedance characteristics, resulting in a major reduction in peak impact force and settling time.

\subsection{Future Work}

There are a number of opportunities to improve both autonomous navigation and manipulation for their incorporation into the decommissioning process. With respect to navigation, implementing ICP navigation techniques in $\mathrm{C}++$ could greatly improved the runtime for the navigation routine, and could also provide versatility in implementation across various platforms. Utilizing effective ICP variants could improve the rate and reliability of convergence, such as the mechanical scanning imaging sonar probabilistic iterative correspondence method, metric-based iterative closest point method, and other high-speed variants. With respect to manipulation, finite element analysis should be conducted on the current iteration of Vidrill in order to identify failure modes and load limits. Fabricating a metal version of Virdrill would allow for strength requirements to be tested for validation. Characterizing the non-linear aspects of Dexter ASV is crucial for developing an accurate model of the system for its use in creating a passive impedance control law. Once this is accomplished, determining impedance ranges that maintain stability for the raft-manipulator system as a whole could shed light on the extent to which autonomous interaction tasks are improved using passivity concepts. These next steps help to pave the path towards a future where the lives of commercial divers are not risked, millions of dollars on support vessels are not wasted, and autonomous underwater vehicles provide new opportunities for the field of decommissioning to keep the environment safe. 


\section{Bibliography}

[1] Albers, Albert, et al. "Semi-autonomous flying robot for physical interaction with environment." Robotics Automation and Mechatronics (RAM). IEEE, 2010.

[2] Barsky, Steven M., and Tom S. Neuman. Investigating recreational and commercial diving accidents. Hammerhead Press, 2003.

[3] Beckwith, Robin. "Under Pressure: Diving's Impact on the Offshore Petroleum Industry." Journal of Petroleum Technology 64.04 (2012): 52-60.

[4] Benjamin, Michael R., Dr. "MOOS-IvP Autonomy Tools Users Manual." 1 May 2013. PDF file.

[5] Besl, Paul J., and Neil D. McKay. "Method for registration of 3-D shapes." Robotics-DL tentative. International Society for Optics and Photonics, 1992.

[6] BP ROV Struggles With Connecter. YouTube. Google, 2 Sept. 2010. Web. 13 Aug. 2015.

$<$ https://www.youtube.com/watch?v=XwrtjEbBoi4 $>$.

[7] Btyner. 3D Tree. 20 Feb. 2006. png file.

[8] Burguera, Antoni, Yolanda Gonzá, and Gabriel Oliver. "Probabilistic sonar scan matching for robust localization." Robotics and Automation, 2007. IEEE, 2007.

[9] Byrd, Robert C., Dr., Donald J. Miller, and Steven M. Wiese. Cost Estimating for Offshore Oil \& Gas Facility Decommissioning. Technical rept. no. EST.1648. Morgantown: AACE, 2014. Print.

[10] Carl Langer and Associates. Analysis and Guidelines for Deepwater Risers. Rept. no. PR-288-03114. Falls Church: Pipeline Research Council International, 2005. Print.

[11] CASE-JIP. Gulf Eddy Model (GEM). Vers. 4.2. Camden: Forristall Ocean Engineering, Inc., 2007. CD-ROM.

[12] Chevron Energy Technology Company Subsea Group. Subsea Acoustic Positioning and Surveys Manual. Rept. no. 1400. Houston: n.p., 2008. Print.

[13] Chevron Environmental Management Company. Personal interview. 23 June 2014. 
[14] Chevron Image Library. 3D Sonar Scan. 2014. jpeg file.

[15] Chevron Image Library. Pipeline Laser Scan. 2014. jpeg file.

[16] Chevron Image Library. ROV Footage. 2014. jpeg file.

[17] Chevron Subsea Survey and Positioning. Marlin AUV. 5 June 2014. PDF file.

[18] Colgate, James Edward, and Neville Hogan. "Robust control of dynamically interacting systems." International journal of Control 48.1 (1988): 65-88.

[19] "Commercial Diving Safety." Occupational Safety $\&$ Health Administration. United States Department of Labor, n.d. Web. 13 Aug. 2015.

$<$ https://www.osha.gov/archive/oshinfo/priorities/diving.html $>$.

[20] Danmeier (Chevron Metocean), Don. Personal interview. 25 July 2012.

[21] Echoscope 3D Imaging Sonar. Echoscope. CodaOctopus, n.d. Web. 13 Aug. 2015. $<$ http://www.codaoctopus.com/products/echoscope $>$.

[22] "Energy Data." Table. Gulf of Mexico Fact Sheet. U.S. Energy Information Administration, n.d. Web. 13 Aug. 2015.

$<$ http://www.eia.gov/special/gulf_of_mexico/>.

[23] Fadali, M. Sami, Dr. "Discrete-Time State-Space Equations." 22 May 2015. PDF file.

[24] "Fresh Water Submersible Delta Transducer." F/T Sensor: Delta IP68. ATI Industrial Automation, n.d. Web. 13 Aug. 2015.

$<$ http://www.ati-ia.com/products/ft/ft_models.aspx?id=Delta + IP68 $>$.

[25] Gilmour (Chevron), Bill, Olu Esan (Chevron), and Blake Carter (Chevron). "ETC Subsea \& MIT Marine Robotics - Meeting Minutes." Memo to Neville Hogan (MIT), John Leonard (MIT), Liam Paull (MIT), and Franz Hover (MIT). Jan. 2015. TS.

[26] "Glossary of NHC Terms." National Weather Service - National Hurricane Center. NOAA, n.d. Web. 13 Aug. 2015.

$<$ http://www.nhc.noaa.gov/aboutgloss.shtml \#h>.

[27] Gorban, A. N., and A. Y. Zinovyev. "Principal Graphs and Manifolds." Handbook of Research on Machine Learning Applications and Trends: Algorithms, Methods and Techniques (2009): 28-59. Print.

[28] HEMedia. "Brent Alpha Oil Rig." The Courier. N.p., 16 Feb. 2015. Web. 13 Aug. 2015.

$<$ http://www.thecourier.co.uk/news/politics/dundee-perfectly-placed-for-brentoil-platform-decommissioning-jobs-1.839192>. 
[29] Hernández, Emili, et al. "MSISpIC: A probabilistic scan matching algorithm using a mechanical scanned imaging sonar." Journal of Physical Agents 3.1 (2009): $3-12$.

[30] Hogan, Neville. "Impedance control: An approach to manipulation." American Control Conference, 1984. IEEE, 1984.

[31] "Hurricanes and the Oil and Natural Gas Industry Preparations." Energy API. American Petroleum Institute, n.d. Web. 13 Aug. 2015.

$<$ http://www.api.org/news-and-media/hurricane-information/hurricanepreparation $>$.

[32] HX Series Environmentally Sealed Position Transducer. UniMeasure Linear Position Transducers. UniMeasure, n.d. Web. 13 Aug. 2015.

$<\mathrm{http}: / /$ www.unimeasure.com/hx.htm $>$.

[33] Kaiser, Mark J. "The impact of extreme weather on offshore production in the Gulf of Mexico." Applied Mathematical Modelling 32.10 (2008): 1996-2018.

[34] Kjer, Hans Martin, and Jakob Wilm. "Evaluation of Surface Registration Algorithms for PET Motion Correction." Diss. Technical University of Denmark, 2010. Print.

[35] Kondak, Konstantin, et al. "Closed-loop behavior of an autonomous helicopter equipped with a robotic arm for aerial manipulation tasks." International Journal of Advanced Robotic Systems 10.145 (2013).

[36] Korpela, Christopher M., Todd W. Danko, and Paul Y. Oh. "MM-UAV: Mobile Manipulating Unmanned Aerial Vehicle." Journal of Intelligent \& Robotic Systems 65.1-4 (2012): 93-101. Print.

[37] Light Duty Rod Actuator. Light Duty Rod Actuators. Firgelli Automations, n.d. Web. 13 Aug. 2015. <https://www.firgelliauto.com/products/light-duty-rod-actuator $>$.

[38] Lockheed Martin. "BM3-D." 2014. txt file.

[39] Maurelli, Francesco, et al. "Investigation of portability of space docking techniques for autonomous underwater docking." Oceans 2009-Europe. IEEE, 2009.

[40] McQueeney, Kerry. "Oil rig diver cheats death at $260 \mathrm{ft}$ as air supply is cut for 40 minutes in the freezing North Sea." Daily Mail. Associated Newspapers, 25 Sept. 2012. Web. 13 Aug. 2015.

<http://www.dailymail.co.uk/news/ article-2208273/ Chris-Lemons-Oil-rigdiver-cheats-death-air-supply-cut-40-minutes-freezing-North- Sea.html $>$.

[41] Mills, Vanessa. "Deep sea diver escapes death after North West Shelf accident." ABC Kimberley. ABC, 5 Apr. 2011. Web. 13 Aug. 2015.

$<$ http://www.abc.net.au/local/stories/2011/04/05/3182971.htm>. 
[42] Minguez, Javier, Luis Montesano, and Florent Lamiraux. "Metric-based iterative closest point scan matching for sensor displacement estimation." Robotics, IEEE Transactions on 22.5 (2006): 1047-1054.

[43] Montesano, Luis, Javier Minguez, and Luis Montano. "Probabilistic scan matching for motion estimation in unstructured environments." Intelligent Robots and Systems, 2005.(IROS). IEEE, 2005.

[44] Park, Hyeonjun, et al. "Dual arm peg-in-hole assembly with a programmed compliant system." Ubiquitous Robots and Ambient Intelligence (URAI). IEEE, 2014.

[45] "Platform Locations in the Gulf." Table. Bureau of Safety and Environmental Enforcement. United States Department of the Interior, 1 Aug. 2015. Web. 13 Aug. 2015.

<http://www.data.bsee.gov/homepg/data_center/platform/platform.asp >.

[46] Ridao, Pere, et al. "Intervention AUVs: The Next Challenge." IFAC world Congress. Keynote in the Marine Robotics Workshop. 2014.

[47] ROV Services Rate Schedule Gulf of Mexico. Portsmouth: Oceaneering International, Inc., 2012. Print.

[48] SDC2130. SDC21xx Brushed DC Motor Controller. RoboteQ, n.d. Web. 12 Aug. 2015.

$<$ http://www.roboteq.com/index.php/roboteq-products-and-services/brusheddc-motor-controllers/sdc2130-detail $>$.

[49] "Side Scan Sonar." Office of Coast Survey. NOAA Office of Coast Survey, n.d. Web. 13 Aug. 2015.

<http://www.nauticalcharts.noaa.gov/hsd/SSS.html >.

[50] Slotine, Jean-Jacques E., and Weiping Li. Applied nonlinear control. Vol. 199. No. 1. Englemonood Clifrs, NJ: Prentice-hall, 1991.

[51] Tangirala, Sekhar, et al. "Feature-Based Navigation for a Platform Inspection AUV." 2011. PDF file.

[52] Tanimoto, Mitsutaka, et al. "Augmentation of safety in teleoperation system for intravascular neurosurgery: a new control strategy for force display based on the variable impedance characterization." Robotics and Automation, 1998. Proceedings. Vol. 4. IEEE, 1998.

[53] Techet, A. H., Prof. "13.012 Hydrodynamics for Ocean Engineers." File last modified on 30 Nov. 2004. PDF file.

[54] Tripod with Biofouling. Woods Hole Coastal and Marine Science Center. USGS, 14 Nov. 2015. Web. 13 Aug. 2015.

<http://woodshole.er.usgs.gov/operations/stg/Gear/tripod.htm>. 
[55] "ULS-100 Scan Performance." 2G Robotics. N.p., n.d. Web. 13 Aug. 2015. $<$ http://www.2grobotics.com/products/underwater-laser-scanner-uls-100/>.

[56] Whitcomb, Louis L. "Underwater robotics: Out of the research laboratory and into the field." Robotics and Automation, 2000. Proceedings. ICRA'00. Vol. 1. IEEE, 2000.

[57] Whitcomb, Louis, Dana Yoerger, and Hanumant Singh. "Advances in Dopplerbased navigation of underwater robotic vehicles." Robotics and Automation, 1999. Proceedings. Vol. 1. IEEE, 1999.

[58] Woodman, Oliver J. An Introduction to Inertial Navigation. Technical rept. no. 696. Cambridge: University of Cambridge Computer Laboratory, 2007. Print. 